\title{
WestVirginiaUniversity
}

THE RESEARCH REPOSITORY @ WVU

Graduate Theses, Dissertations, and Problem Reports

2017

\section{Finite Element Simulation of Large-Scale Confined Inflatable Structures}

Iole Pecora

Follow this and additional works at: https://researchrepository.wvu.edu/etd

\section{Recommended Citation}

Pecora, lole, "Finite Element Simulation of Large-Scale Confined Inflatable Structures" (2017). Graduate Theses, Dissertations, and Problem Reports. 6392.

https://researchrepository.wvu.edu/etd/6392

This Thesis is protected by copyright and/or related rights. It has been brought to you by the The Research Repository @ WVU with permission from the rights-holder(s). You are free to use this Thesis in any way that is permitted by the copyright and related rights legislation that applies to your use. For other uses you must obtain permission from the rights-holder(s) directly, unless additional rights are indicated by a Creative Commons license in the record and/ or on the work itself. This Thesis has been accepted for inclusion in WVU Graduate Theses, Dissertations, and Problem Reports collection by an authorized administrator of The Research Repository @ WVU. For more information, please contact researchrepository@mail.wvu.edu. 


\title{
FINITE ELEMENT SIMULATION OF LARGE-SCALE CONFINED INFLATABLE STRUCTURES
}

\author{
Iole Pecora \\ Thesis submitted to the \\ Benjamin M. Statler College of Engineering and Mineral Resources \\ at West Virginia University \\ in partial fulfillment of the requirements for the degree of \\ Master of Sciences \\ in \\ Mechanical Engineering
}

Eduardo M. Sosa, Ph.D., Chair

Gregory J. Thompson, Ph.D.

Ever J. Barbero, Ph.D.

Department of Mechanical and Aerospace Engineering

\author{
Morgantown, West Virginia \\ 2017
}

Keywords: Abaqus, Deflation, Deployment, Finite Element Simulation, Folding, Inflation, Membrane, Tunnel, Inflatable Structures.

Copyright @ 2017 Iole Pecora 


\section{Abstract \\ Finite Element Simulation of Large-Scale Confined Inflatable Structures}

\section{Iole Pecora}

The protection of transportation tunnels is one of the top priorities of transportation and government entities. Transportation tunnels have been identified as particularly vulnerable to different threats such as propagation of toxic gases, or smoke originated by human activities or flooding originated by extreme climatic events such as hurricanes and severe weather. Finding solutions to minimize the consequences of disastrous events has become critical to increase the resiliency of tunnel systems. The implementation of large-scale inflatable structures at specific locations of the tunnel system for containing the propagation of flooding or gases is now possible. When a threat happens, a sensing system detects the threat and triggers the activation of an inflation system which can deploy, inflate and pressurize the inflatable structure in a few minutes. When the inflatable structure is completely inflated, it acts as a barrier that can isolate the compromised region and contain the threat. The feasibility of this concept was demonstrated in 2008, and several experimental evaluations were conducted in the recent years to demonstrate the operational viability of this solution. Despite the successful results seen in the experimental evaluations, the development of simulations that can predict results in advance to reduce the number of experimental iterations is still essential. Finite Element simulation efforts performed in the recent years contributed to the understanding of the dynamics of the deployment and inflation of an inflatable structure for one particular tunnel profile and one folding and deployment configuration. However, if the membrane material of the inflatable changes, or the shape or configuration of the tunnel profile changes, or the position for storage of the folded inflatable changes, the initial behavior of the unstressed membrane during the initial deployment and later inflation, will be different. All this variability increases the need of experimental iterations to determine the appropriate combination of parameters to achieve acceptable results. Considering that the resources for experimental iterations can be very limited, there is a clear need to continue with the development of predictive models that can account for the different factors involved in the implementation of inflatable structures for tunnel protection.

This work presents the development of Finite Element simulations generated for the evaluation of different phases of the operation of a large-scale inflatable structure used for sealing a tunnel segment. The simulations developed in this work focused on reproducing deflation, folding, and placement procedures for deploying an inflatable from the ceiling of a tunnel segment. The models were also used to evaluate the behavior of the inflatable during the initial deployment and the full inflation. Different strategies were analyzed with the ultimate goal of maximizing the global and local conformity, which translate in a better 
sealing capacity of the inflatable to the tunnel profile. The results of the simulations showed that a very flat shape can be achieved by implementing a controlled deflation of the nominal shape of the inflatable as a starting point of the folding procedures. Moreover, a combination of translational and rotational planes allowed the flattened shape to reach a more compact shape at the end of the folding procedures. Simulation results also showed that the stiffness of the membrane influenced the shape and behavior of the inflatable during the initial deployment. Moreover, results demonstrated that the implementation of passive restrainers to control the movement and release of the membrane during the deflation, folding, deployment and inflation contributed to reach higher levels of local conformity of the inflatable to the tunnel perimeter, as well as an increase of the contact area as the global and local conformity improved. A comparison of simulation results with available experimental data demonstrated a good level of agreement between the finite element simulations and the experimental observations. 
To my father 


\section{Acknowledgments}

I would like to thank my research advisor, Dr. Eduardo M. Sosa, for his guidance and support throughout this project. I would like to thank him too for his time and technical advice.

I would also like to thank the committee members Dr. Ever J. Barbero and Dr. Gregory J. Thompson for providing me helpful comments and for their time reviewing my thesis.

Finally, I would like to thank Stéphane and my family who always supported and encouraged me. Thank you for your love. 


\section{Table of Contents}

1 Chapter 1. Introduction ........................................................................................................................ 1

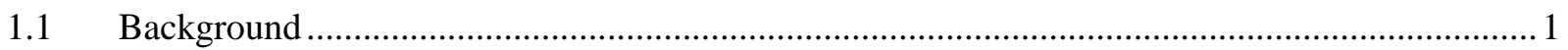

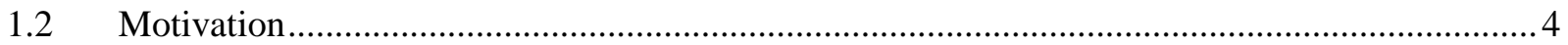

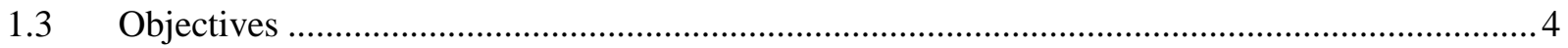

$1.4 \quad$ Outline

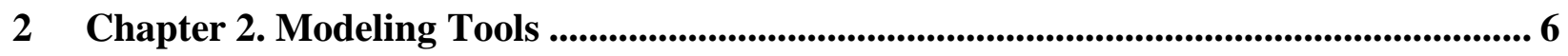

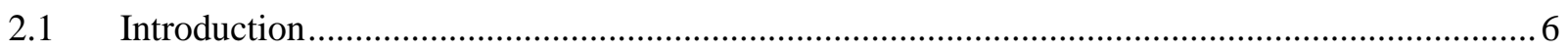

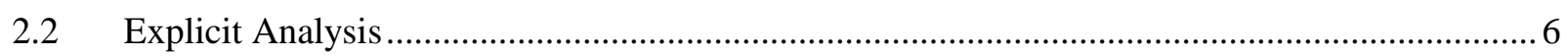

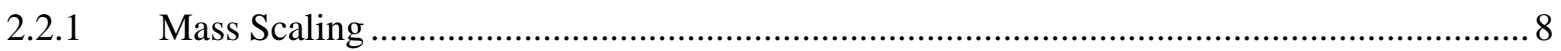

2.3 Overview of Element Types Implemented in this Work .........................................................

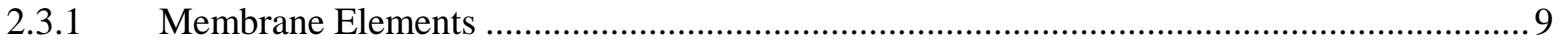

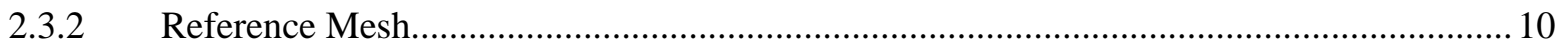

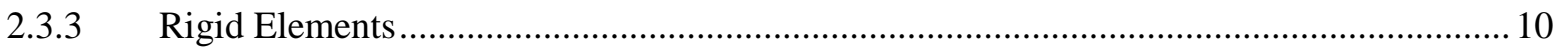

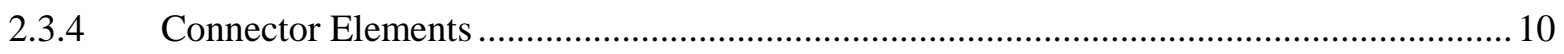

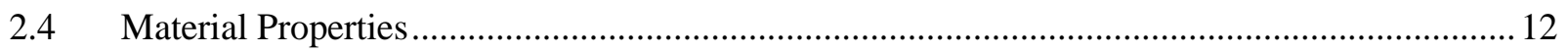

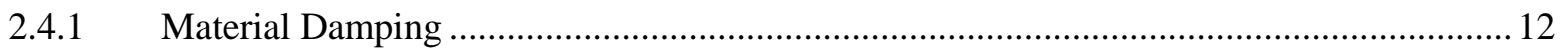

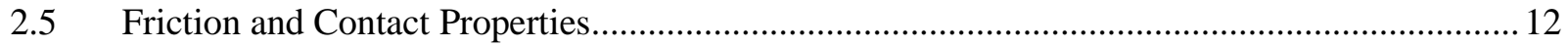

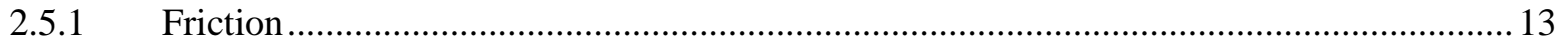

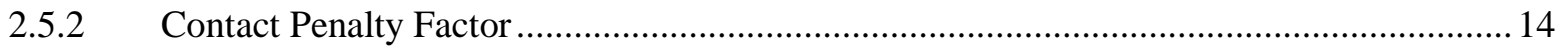

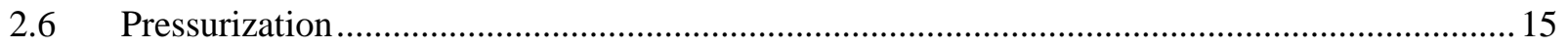

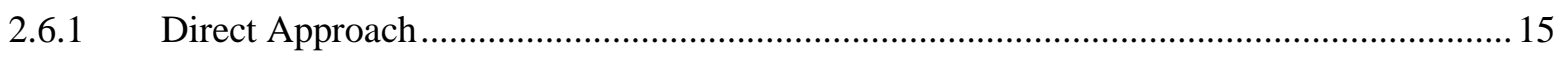

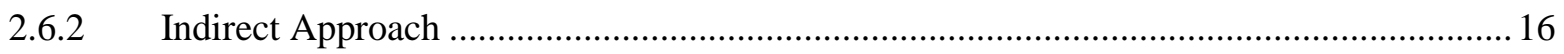

3 Chapter 3. Model Generation........................................................................................ 19

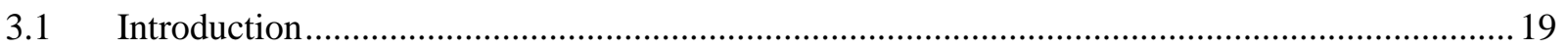

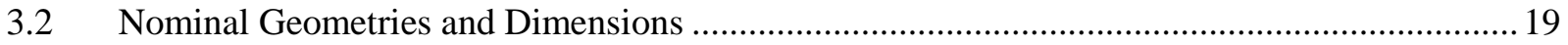

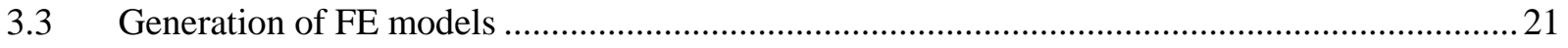




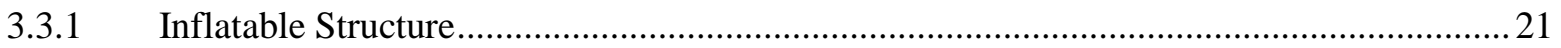

3.3.2 Stress Evaluation and Mesh Convergence Study ........................................................ 23

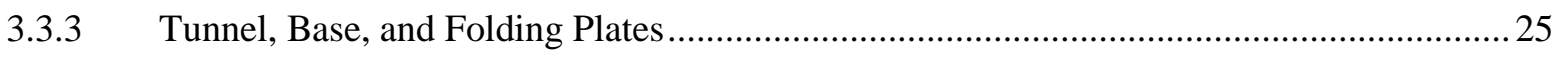

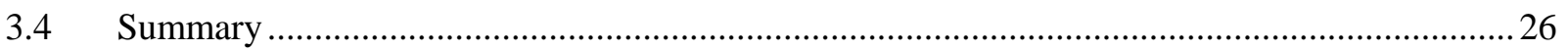

4 Chapter 4. Deflation and Unconfined Inflation .................................................................. 27

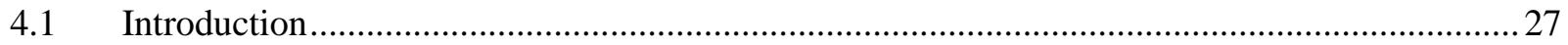

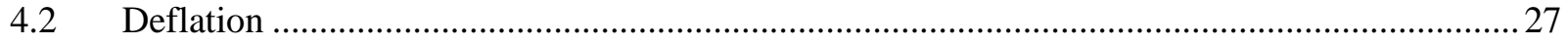

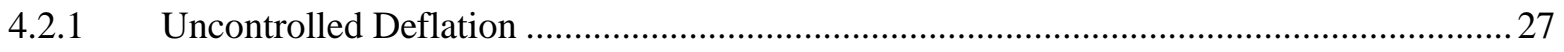

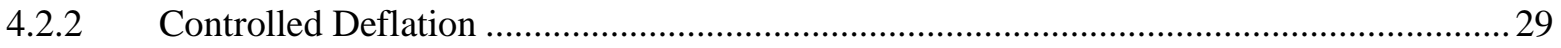

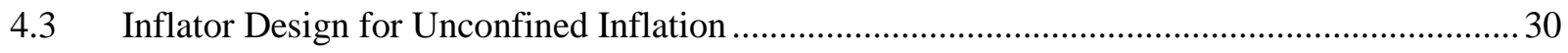

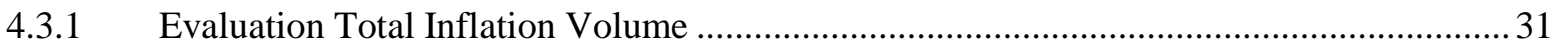

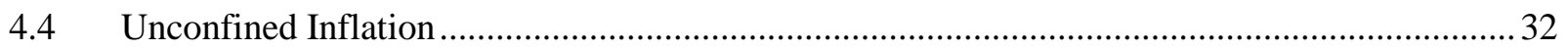

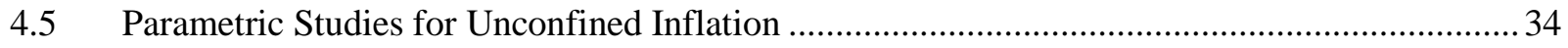

4.5.1 Parametric Study on Mass Scale Factor ........................................................................ 34

4.5.2 Parametric Study on Influence of Mass Proportional Damping Factor $\boldsymbol{\alpha}$.......................... 40

4.5.3 Influence of Ambient Temperature Ta during Unconfined Inflation .............................. 41

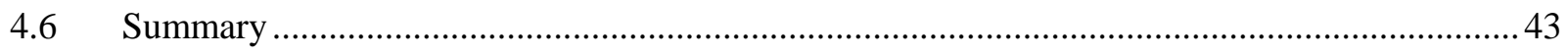

5 Chapter 5. Confined Inflation - Uncontrolled Membrane Release ................................. 45

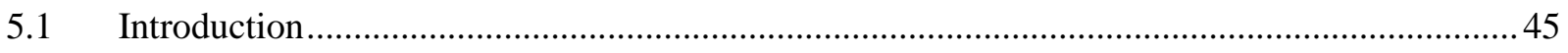

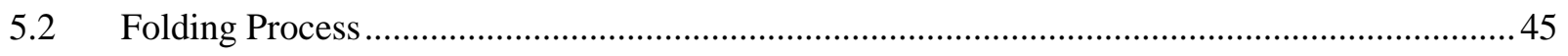

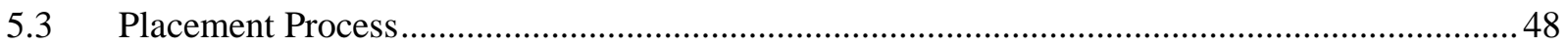

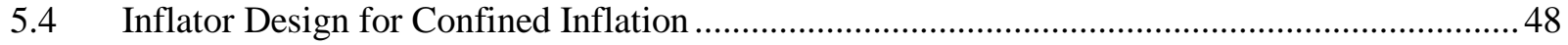

5.5 Initial Deployment and Confined Inflation ....................................................................... 49

5.5.1 Influence of Stiffness of the Membrane during Unconfined Inflation.............................52

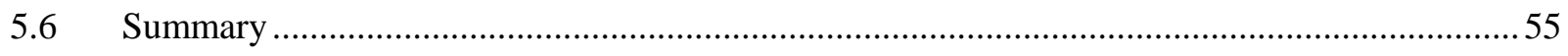

6 Chapter 6. Confined Inflation - Controlled Membrane Release ..................................... 56

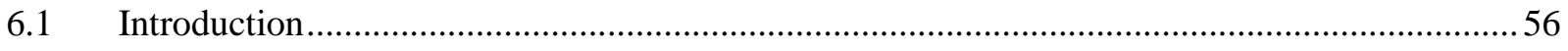


6.2 Controlled Deflation Including Pre-folding Steps ........................................................56

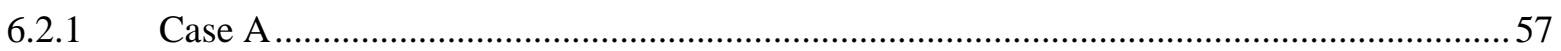

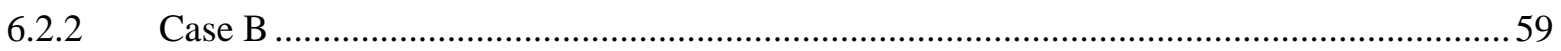

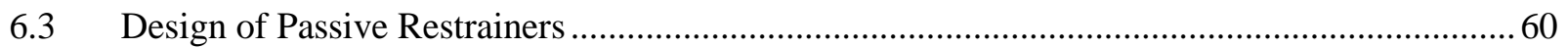

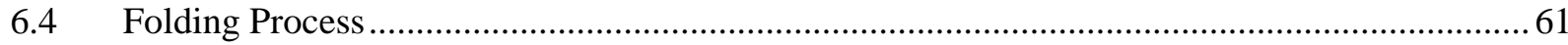

6.5 Confined Inflation with Controlled Release of Membrane .....................................................64

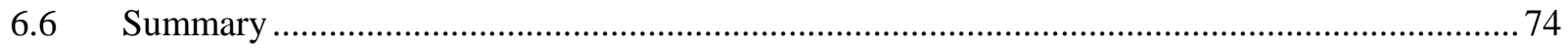

7 Chapter 7. Conclusions and Recommendations for Future Work .............................. 76

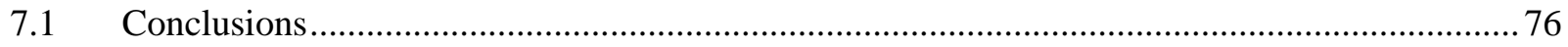

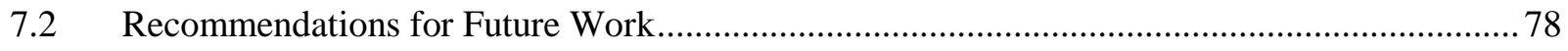

8 Appendix A. Deployment and Inflation of a Segment of Inflatable Boom..................... 80

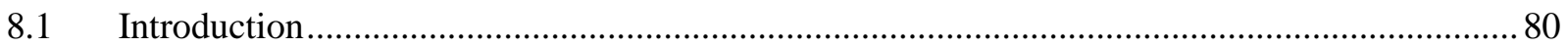

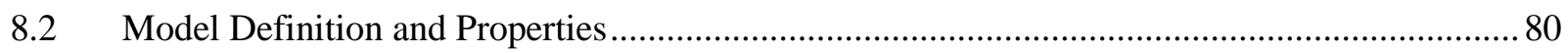

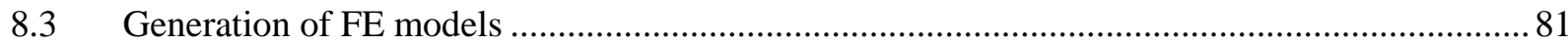

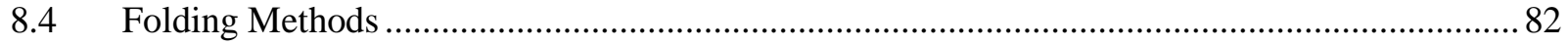

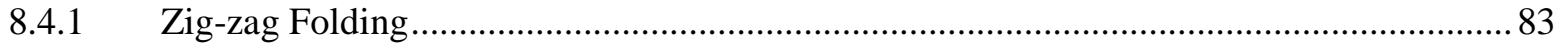

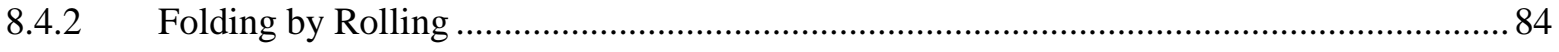

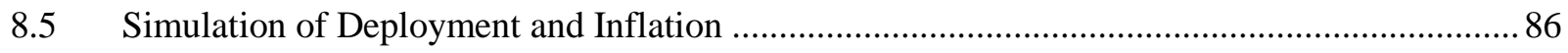

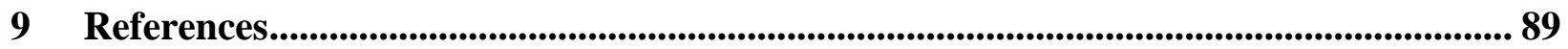




\section{List of Figures}

Figure 1.1. Full -Scale Experiment [7] .................................................................................... 1

Figure 2.1. Normal orientation for membrane elements [27]. ......................................................... 9

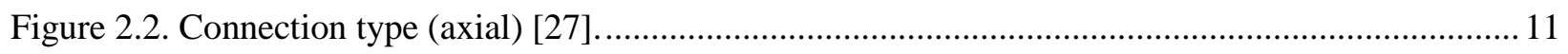

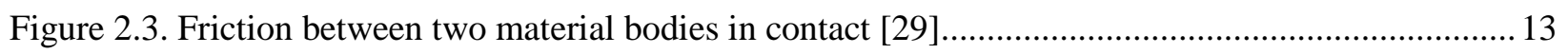

Figure 3.1. Inflatable structure geometry and dimensions (dimensions in meters). ................................20

Figure 3.2. Tunnel cross-section dimensions (dimensions in meters). ................................................20

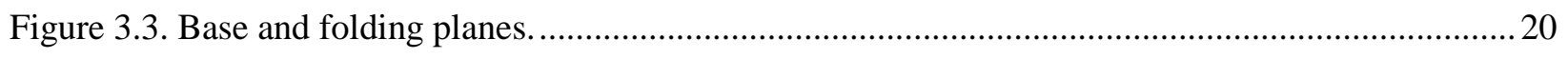

Figure 3.4. Inflatable structure, FE initial geometry, and partitions generated using Abaqus/CAE..........21

Figure 3.5. Constitutive model of fabric material: (a) Mechanical behavior under tensile load; (b)

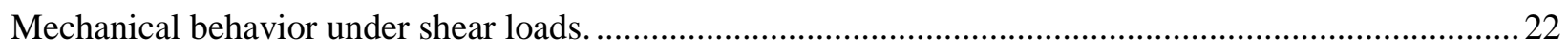

Figure 3.6. Stress distribution contour for Mesh A, Mesh B, and Mesh C.............................................2 24

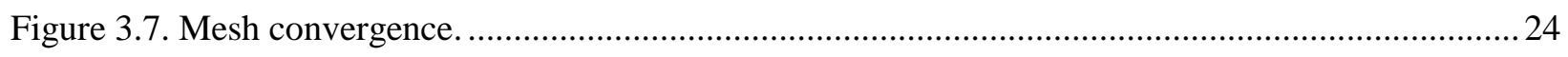

Figure 3.8. Meshes of folding planes, base and tunnel segment.......................................................25

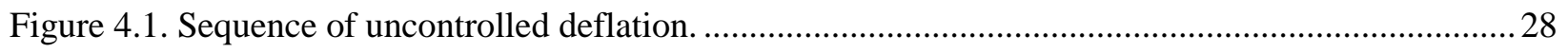

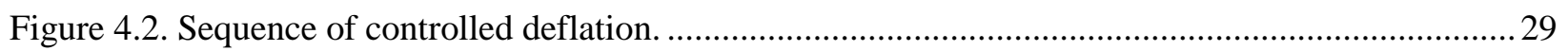

Figure 4.3. Flat shape after controlled deflation and application of gravity load. ..................................... 30

Figure 4.4. (a) Air mass flow rate; (b) Time history of thermodynamic properties using the initial inflator.

Figure 4.5. Time history of thermodynamic properties using the adjusted inflator.................................. 34

Figure 4.6. Mass Scale factor (MSF) vs. Computational Time. ............................................................. 35

Figure 4.7. Time history of kinetic energy for different values of MSF................................................ 37

Figure 4.8. Time history of acceleration for different values of MSF . .................................................. 37

Figure 4.9. Time history of $\boldsymbol{S 1 1}$ on the cylindrical portion at different values of MSF......................... 39

Figure 4.10. Time history of $\boldsymbol{S} \mathbf{2 2}$ on the cylindrical portion at different values of MSF......................... 39

Figure 4.11. Time history of $\boldsymbol{S 1 1}=\boldsymbol{S 2 2}$ on the tip of spherical portion at different values of MSF........39

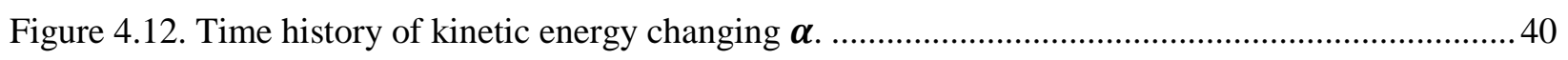

Figure 4.13. Adiabatic and isothermal transformation on the Clapeyron plane at $\boldsymbol{T a}=\mathbf{1 5}^{\circ} \boldsymbol{C} \ldots \ldots \ldots \ldots \ldots . . . . .42$

Figure 4.14. Isothermal transformations on the Clapeyron plane for increasing $\mathbf{T a}$............................. 42

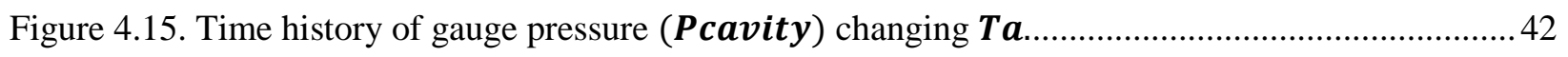

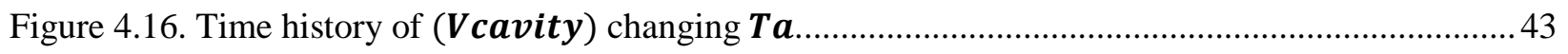

Figure 5.1. Folding sequence, main folding steps, top view (folding planes removed for clarity).............45

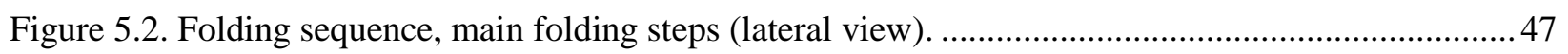




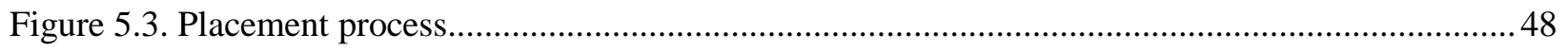

Figure 5.4. Detail of attached lines in the ceiling of the tunnel. ..........................................................50

Figure 5.5. Results of FE Model compared to full-scale experiments reported in [7].............................50

Figure 5.6. FE Model: (a) Detail of wrinkles on the tunnel floor; (b) Detail view of local conformity. ....51

Figure 5.7. Time history of gauge pressure and internal volume for an air mass flow rate of $0.66 \mathrm{~kg} / \mathrm{sec} .52$

Figure 5.8. Time history of strain energy for different values of artificial compressive strength. ............53

Figure 5.9. Comparison of simulation results for Cases 1, 3 and 6 vs. experimental results....................54

Figure 6.1. Controlled deflation, reference lines and position of pre-folds, Case A.................................58

Figure 6.2. Sequence of controlled deflation with pre-folding steps Case A..........................................58

Figure 6.3. Controlled deflation, reference lines and position of pre-folds, Case B................................59

Figure 6.4. Sequence of controlled deflation with pre-folding steps Case B.........................................59

Figure 6.5. Folding sequence, main folding steps, top and isometric views.........................................61

Figure 6.6. Lateral view of the folding sequence corresponding to Cases A and B..............................63

Figure 6.7. (a) Folded shape without pre-folds (Case 0); (b) Folded shape including pre-folds (Cases A and B).

Figure 6.8. Comparison of simulation results for Case A and Case B vs. experimental results................65

Figure 6.9. Release of the membrane. Comparison of simulation results for Case 0, A and Case B. ........66

Figure 6.10. Radii of curvature of inflatable at the onset of failure of passive restrainers. ......................67

Figure 6.11. Time history of gauge pressure, internal volume and failure status for case A....................6 68

Figure 6.12. Time history of gauge pressure, internal volume and failure status for case B....................69

Figure 6.13. Time history of axial force in each connector for case A................................................... 70

Figure 6.14. Time history of axial force in each connector for case B . .............................................. 71

Figure 6.15. Time history of contact area for the three cases (Case 0, Case A, Case B),........................ 72

Figure 6.16. Comparison of vacuum effect for the three cases (Case 0, Case A, and Case B)..................74

Figure 8.1. (a) Inflatable boom geometry and dimensions; (b) Partitions. ................................................ 80

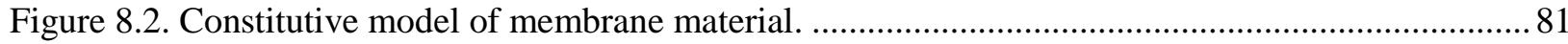

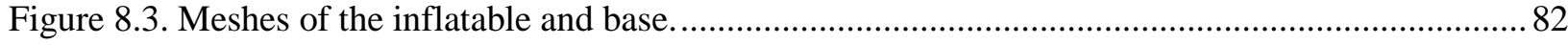

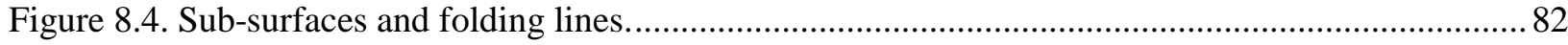

Figure 8.5. Two zig-zag folding sequence, main folding steps. ............................................................ 84

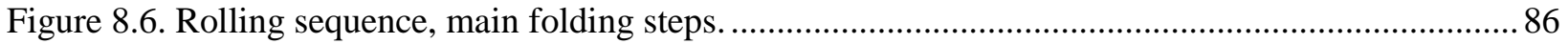

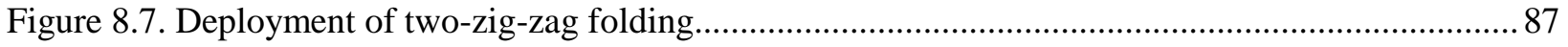

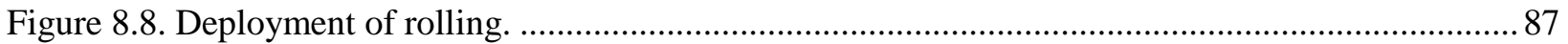

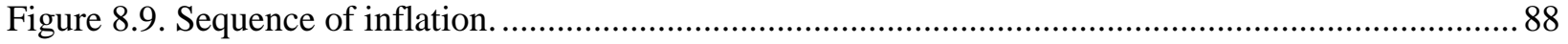




\section{List of Tables}

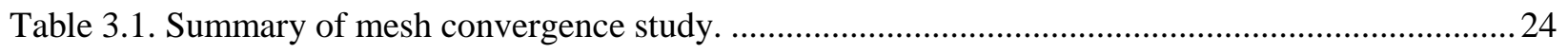

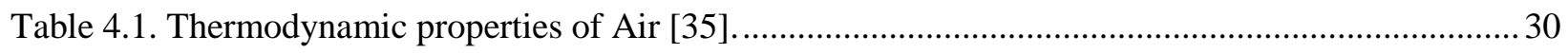

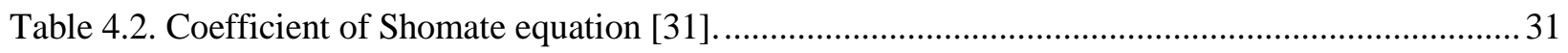

Table 4.3. Maximum values of the stresses in select nodes for changing MSFs. ..................................... 38

Table 6.1. Percentage of improvement of the contact area. ................................................................. 73

Table 6.2. Values of vacuum pressure and internal volume during initial deployment........................... 73 


\section{Chapter 1. Introduction}

\subsection{Background}

Tunnel safety has become a great concern for transportation and government entities in the last decades. [1-3]. Transportation tunnels have been identified as particularly vulnerable to different threats such as propagation of toxic gases, or smoke originated by human activities or flooding originated by extreme climatic events [4-6]. Finding solutions to minimize the consequences of disastrous events has become critical to increase the resiliency of transportation tunnel systems. One possible solution to contain the propagation of flooding or gases is the implementation of large-scale inflatable structures at specific locations of the tunnel system. When a threat happens, a sensing system detects it and triggers the activation of an inflation system which can deploy, inflate and pressurize the inflatable structure in a few minutes. When the inflatable structure is completely inflated, it acts as a barrier held mostly by friction and isolates the compromised region to contain the threat. When the threat is mitigated, the structure is deflated, folded, repacked and removed from the compromised zone allowing repairs and maintenance of the affected area and the installation of a new folded and packed inflatable. The feasibility of this concept was tested in 2008 in full-scale setup using an inflatable manufactured from a single-layer fabric material, as shown in Figure 1.1. In that test, the inflatable was deployed from the ceiling of a service tunnel and then inflated with air at a low pressure in approximately three minutes [7].

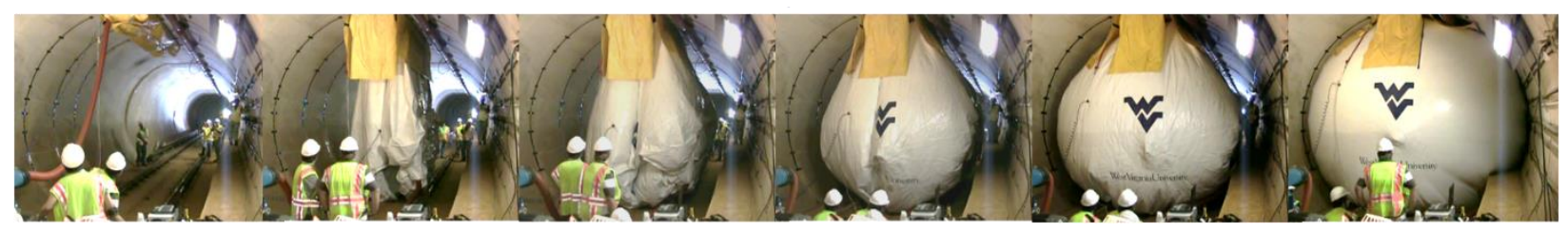

Figure 1.1. Full -Scale Experiment [7].

The design, manufacturing, and testing of large-scale inflatables for tunnel protection brought new challenges, due not only to the large scale of the problem but also to the complexity of the tunnel profile to which the inflatable had to conform. The design and the reliability of the parameters involved in the design of this type of structure were addressed in $[8,9]$. Moreover, in the last few years, extensive experimental evaluations were conducted to evaluate and understand aspects of the operation and mechanical behavior of large-scale inflatables. The feasibility of using a more robust membrane as well as the implementation of a lateral deployment instead of a ceiling deployment was evaluated in [10, 11]. The evaluation of frictional characteristics of the inflatable when subject to flooding pressures were evaluated in reduced scale experiments reported in [12]. A key aspect found in the experimental work was that the level of conformity 
of the inflatable to the tunnel perimeter plays an important role in the sealing capacity of the inflatable. Experimental investigations of the initial deployment of large-scale structures showed that the initial shape achieved by the inflatable at the end of the initial deployment and inflation at low pressure is critical for maximizing the sealing capacity [13]. The presence of gaps in the contact between the inflatable and the inner tunnel surfaces not only reduces the frictional capacity to remain stable when subject to the external pressure but also increases the sources and amount of leakage that the inflatable is meant to contain [13].

From the operational point of view, experimental tests showed that the implementation of a large-scale inflatable for sealing one or more segments of a tunnel system could be divided into three main phases [9, 13]: Phase 1, preparation and installation; Phase 2, initial deployment and inflation at low pressure; Phase 3 , pressurization of the inflatable structure to contain the pressure of the threat, either gas or water. In Phase 1 , the inflatable structure is folded and placed within a portable container that is then transported to a specific location of the tunnel segment and pre-installed. Phase 2 starts when a sensing system detects a threatening event. The sensing system activates the automatic opening of the container allowing the initial unfolding and deployment followed by the activation of inflation system. When the inflatable is in place, Phase 3 starts with the pressurization to ensure that the inflatable will remain in place when subject to the external pressures.

Unlike the inflatable structures used in automobile and aerospace applications, which are relatively small and lightweight, the inflatables for protection of tunnels can have large dimensions and high weight due to the robustness needed in the fabric material to withstand the internal as well as the external pressures [13]. Experimental work carried out with full-scale prototypes demonstrated to be labor intensive and required multiple iterations to achieve consistent results. Moreover, experimental results reported in [11, 13] indicated that the preparation procedures including the deflation, folding sequence and position of the deployment have an influence on how the membrane of the inflatable behaves during the initial deployment and then how it conforms to the tunnel section at the completion of inflation.

Wong, 2013 [14] carried out the first effort to simulate the maneuvers corresponding to the folding, placement in the tunnel, deployment from a lateral position, and inflation. Wong adopted techniques applied in the automobile and aerospace application such as folding by rolling as well as the installation of passive restrainers to control the release of the membrane during the initial deployment $[14,15]$. In his models, the fabric material of the inflatable was modeled as an orthotropic material, including shear resistance and frictional properties. Wong also adopted an inflator originally designed to simulate the inflation of automobile airbags [16-18]. The results reported by Wong, 2013 [14] matched reasonably well the experimental observations reported in $[10,11,13]$ and provided a starting point that can be used as a reference for simulating similar inflatable structures installed in other tunnel configurations. 
On the other hand, inflatable structures are usually folded following two patterns: zig-zag folds (or z-folds) and rolling. These two patterns are commonly used in the automobile industry for folding driver's and passenger's airbags, and also in the aerospace industry for folding inflatable antennas, membrane reflectors or toroidal rims [19-25]. In the simulation of these two patterns, the inflatable is typically flattened first and then folded using a combination of folding rigid planes pre-positioned at specific locations that translate or rotate following specific paths defined depending on the particular shape to be achieved [19, 23]. Similar techniques have been used to reproduce the manual operations implemented experimentally for folding large-scale inflatable structures used for sealing tunnels [14, 15].

Experimental observations showed that the membrane of the inflatable is unstressed during the initial deployment, or at most, subject to a load corresponding to its weight $[11,13]$. One of the challenges for modeling this behavior is the simulation of the stiffness and wrinkles for unstressed conditions. In the finite element simulations, it is common to use membrane elements to represent the performance inflatable structures. However, membrane elements can become unstable when subjected to in-plane compressive stresses. Since the mechanical behavior of membranes is assumed to have no resistance under compression, when the membrane is subjected to compressive stresses, it tends to avoid compressive stresses by out of plane deformation called wrinkling. One way to overcome this problem and in order to represent large deformations of thin membranes, a pseudo-surface can be defined. This pseudo-surface represents the global surface of the membrane without wrinkles, and the membrane is defined in a three-dimensional space with the surface in plane stress state. With this wrinkling procedure, the membrane is considered to deform from its non-deformed configuration to the deformed configuration [26]. Additionally, it is common in the simulation of airbags to assign a very small artificial compressive strength to the fabric material in order to reduce excessive distortion of the membrane elements [27].

Furthermore, two inflation models are typically adopted for the simulation of the inflation of inflatables. One of the simplest models, originally designed for simulation of deployment and inflation of automobile airbags, is the Control Volume (CV) method, also known as the Uniform Pressure Method (UPM) originally proposed by Wang, 1988 [16-18]. This method was implemented in the simulations developed by Wong, 2013 [13] with results in good agreement with the experimental observations reported in [10, 11, 13]. A second and more sophisticated model is the Coupled Eulerian-Lagrangian (CEL) method [18, 27]. This second method is also used in the simulation of automobile airbags and provides the ability to model the dynamics of the gas flow in the airbag and to include the effects of surrounding air during deployment [27]. This method demonstrated to be more accurate for reproducing very fast-occurring events (in the order of milliseconds) but at a high computational cost. 


\subsection{Motivation}

The experimental work reported in the literature demonstrated that the evaluation of feasibility and operational viability of the implementing large-scale inflatable structures for the protection of tunnels could be complex, effort-intensive and time-consuming. Despite the successful results seen in the experimental evaluations, the development of simulations that can predict results in advance in order to reduce the number of experimental iterations is essential. The efforts initiated by Wong, 2013 [14] served to understand the dynamics of the deployment and inflation of an inflatable structure for one particular tunnel profile and one folding and deployment configuration. However, if the shape or configuration of the tunnel profile changes, or the position for storage of the folded inflatable changes, the initial behavior of the unstressed membrane during the initial deployment and later inflation will be different. All this variability increases the need of experimental iterations to find the appropriate combination of parameters to achieve acceptable results. Considering that the resources for experimental iterations are limited, there is a clear need to continue with the development of predictive models that can account for the different factors involved in the implementation of inflatable structures for tunnel protection. That need motivated the development of the work presented in this thesis.

\subsection{Objectives}

The main objective of this work is to create Finite Element (FE) models able to simulate the procedures for the preparation and deployment of inflatable structures used for the protection of tunnels. Using the techniques developed by Wong, 2013 [14] as a starting point, this work explores new ways for better control of the membrane since it plays an important role on the final conformity of the inflatable to the tunnel. This work also aims to demonstrate that using a simple geometry for the inflatable it is possible to achieve a higher level of conformity without using a fitted shape of the inflatable adopted in the experiments reported by Martinez, 2008 [7]. The purpose of the FE models is to simulate the following operations:

A. Folding methods that follow the procedures implemented experimentally, including:

- The implementation of a controlled deflation to reach a flat shape.

- The implementation of a folding procedure for the flat shape that minimizes the storage volume.

B. Initial deployment and inflation, which will require:

- The definition of placement procedures of the folded shape in the storage area of the tunnel crosssection.

- The design of an inflator system taking into account the available experimental results.

- The definition of a sequence of deployment and activation of the inflator for inflation under confined conditions. 
C. Parametric studies to evaluate the influence of parameters on the performance of the inflatable such as:

- The stiffness of the membrane during initial deployment.

- Mass scaling factor.

- Mass proportional damping factor.

- Ambient temperature.

- Folding technique with and without the implementation of initial pre-folds.

\subsection{Outline}

This thesis is divided into seven chapters. In Chapter 1, background information about the development of inflatable structures for tunnel protection was presented along with the motivation and main objectives of this work. Chapter 2 provides a brief overview of the formulation and the main tools used for the generation and analysis of the FE models developed in this work. Chapter 3 presents the geometrical properties and set-up of the FE models corresponding to all the parts modeled in this work. Chapter 3 also presents the results of a mesh convergence analysis for the model corresponding to the inflatable structure under unconfined pressurization. In Chapter 4, two techniques of deflation are introduced and discussed: the uncontrolled deflation and the controlled deflation. Chapter 4 also presents the definition of the inflator system implemented in the simulation of unconfined inflation and concludes with a parametric study conducted to understand how changing of parameters influence the behavior of the inflatable structure and also the computational time. Starting from the flattened shape obtained at the end of the controlled deflation, Chapter 5 describes the folding procedure adopted to achieve a compact folded shape, the placement process and the simulation of confined inflation. This chapter also presents an initial comparison between FE simulations and experimental results and concludes with a parametric study performed to evaluate the influence of stiffness of the membrane material on the global behavior during the initial deployment and inflation. Taking into account all the knowledge gained from the results described in the previous chapters, Chapter 6 describes an enhanced technique developed to improve the membrane behavior of the inflatable during the initial deployment and inflation, and to correct the lack of local conformity observed in Chapter 5. Finally, Chapter 7 presents the main conclusions drawn from the results presented in previous chapters and also provides recommendations for future work. 


\section{Chapter 2. Modeling Tools}

\subsection{Introduction}

The Finite Element Method (FEM) is a numerical procedure in which a structure is subdivided into an assembly of a finite number of elements with an assumed form of displacement or stress distribution. The solution is obtained by combining these individual finite displacements or stress distributions in a way that satisfies the force-equilibrium and displacement related to the elements chosen. The FEM is suitable for the analysis of complex structures subjected to complex loading scenario.

The Simulia Finite Element simulation package was implemented in this work [27]. In particular, the geometry and meshing of the model were generated using Abaqus/CAE. All the nodes and the element were later renumbered with HyperMesh [28] tools, and the model properties were compiled in an Abaqus input file (.inp) in order to make the simulation work more efficiently. The Abaqus inp file includes material properties and the mechanical properties needed for the proper definition of the structural model. In this work, all the models were solved with the explicit solution solver, and Abaqus/Viewer was used to visualize and post-process the simulation results.

Starting from the idealization of the structure using the FEM, this section intends to provide the reader with a brief overview of the formulation and the main tools used for the generation and analysis of the models developed in this work.

\subsection{Explicit Analysis}

The explicit solution solver was used in this work to simulate the following: a) Quasi-static behaviors with complicated contact interactions; b) large displacement behavior including large rotations and large deformations, accounting for geometrically non-linear deformations.

The explicit dynamic procedure requires a large number of small time increments to achieve the final configuration resulting from the application of loads [27, 29]. In Abaqus, the explicit solver implements the central-difference time integration rule. The explicit central-difference operator satisfies the dynamic equilibrium equations at the beginning of the increment $t$. The accelerations calculated at time $t$ are used

to advance the velocity solution to time $t+\frac{\Delta t}{2}$ and the displacement solution to time $t+\Delta t$. The velocity and acceleration are approximated by the finite difference method. 
The relations for velocity and displacement are:

$$
\begin{gathered}
\dot{u}_{\left(i+\frac{1}{2}\right)}^{N}=\dot{u}_{\left(i-\frac{1}{2}\right)}^{N}+\frac{\Delta t_{(i+1)}+\Delta t_{(i)}}{2} \ddot{u}_{i}^{N} \\
u_{(i+1)}^{N}=u_{(i)}^{N}+\Delta t_{(i+1)} \dot{u}_{\left(i+\frac{1}{2}\right)}^{N}
\end{gathered}
$$

Where $u^{N}$ is a degree of freedom (displacement or rotation component) and the subscript $i$ refers to the increment number in an explicit step.

The diagonal element mass matrix is used in the explicit procedure to increase the computational efficiency. The accelerations at the beginning of the increment are computed by:

$$
\ddot{u}_{i}^{N}=\left(M^{N J}\right)^{-1}\left(P_{i}^{J}-I_{i}^{J}\right)
$$

Where $M^{N J}$ is the mass matrix, $P^{J}$ is the applied load, and $I^{J}$ is the internal force vector. The velocity and the nodal positions (or displacements) are then calculated by plugging the acceleration calculated in Eq. (2.3) into Equations (2.1) and (2.2). The incremental deformation produces increments in the strains and stresses. Knowing the strains and stresses, it is possible to compute the internal forces.

An approximation to the stability limit can be written as [27, 29]:

$$
\Delta t \approx L_{\min } / c_{d}
$$

Where, $L_{\min }$ is the smallest element dimension in the mesh, and $c_{d}$ is a material property defined as the dilatational wave speed which is typically expressed in terms of $\lambda$ and $\mu$ :

$$
c_{d}=\sqrt{\frac{\lambda+2 \mu}{\rho}}
$$

Where $\lambda$ and $\mathrm{G}=2 \mu$ are the Lamè's constants and $\rho$ is the material density.

The FE models created in this work were subjected to large deformations that produced reductions of the characteristic length of the elements. These reductions resulted in, as shown in the Eq. (2.4), a decrease of the time increment that produced an increase of the computational time.

Scaling the mass of the excessively deformed elements can decrease the computational time. This approach is called mass scaling, and it is summarized next. 


\subsubsection{Mass Scaling}

This approach artificially increases the material density $\rho$ by a factor of $f^{2}$ and it produces a decrease of wave speed $c_{d}$ by a factor of $f$ [27]. From Eq. (2.5) and Eq. (2.4), this increase of density produces an increase of the stable time by a factor of $f$. By increasing the stable time increment through mass scaling, it is possible to analyze the model in its natural time period. The factor $f^{2}$ is known as Mass Scale Factor (MSF) and has exactly the same effect on the inertia forces as accelerating the time of simulation.

The implementation of mass scaling is convenient in rate-dependent problems, but it must be used with care to ensure that the inertial forces do not dominate and change the solution. Indeed, from the Equations 2.1, 2.2 and 2.3, it can be seen that increasing the stable time increment produces an artificial increase of the displacement, velocity, and acceleration that are not representative of the physical reality under consideration.

There is not an exact way to find the exact value of the mass scaling factor. However, it can be found with a relatively low number of iterations. In Abaqus, the mass scaling can be fixed or variable for each step of the simulations [27].

In this work, a fixed mass scaling was implemented. In a quasi-static analysis, the mass scaling factor is usually applied to the entire model once at the beginning of the step. It is important to highlight that the items in a model that are affected by mass scaling are [27]:

- Mass, rotary inertia, rigid, and infinite elements

- Rotary inertia in beams and shells

- Bulk viscosity and mass proportional damping

Moreover, the items in a model that are not affected by mass scaling are [27]:

- Gravity loads

- Adiabatic heat calculations

- Thermal solution response in a fully coupled thermal-stress analysis

- Equation of state materials

- Fluid and fluid link properties

- $\quad$ Spring and dashpot elements

In this work, the relevant items from the list above were the mass, the mass proportional damping $\alpha$ and gravity load which was always active for the duration of the simulations. 


\subsection{Overview of Element Types Implemented in this Work}

The elements used in the FE models created in this work were the membrane element, the rigid element, and the connector element. Membrane elements were used to represent the thin wall of the fabric material that constituted the inflatable structure. Rigid elements were used to represent auxiliary components such as the base in which the model was leaning during some stages of the simulation, as well as the folding planes. Rigid elements were also used to represent the confining environment created by the tunnel walls. Connector elements were used to simulate the holding of the membrane and the controlled release of the membrane during the deployment maneuvers. Additional details for each type of element are presented next.

\subsubsection{Membrane Elements}

The membrane elements are surface elements that transmit in-plane forces only. These elements have no bending stiffness so they cannot carry out bending moments. General membrane elements are used in threedimensional models in which the deformation of structure can evolve in three dimensions. In this work, a three-dimensional triangular membrane element named (M3D3) was implemented for representing the membrane of the inflatable, where " $\mathrm{M}$ " indicates membrane element, "3D" represents the dimensionality of the element, and " 3 " is the number of the nodes in the element [27].

Figure 2.1 shows the convention used in Abaqus to assign the normal direction to the surface of membrane elements. The 'top' surface of a membrane is the surface in the positive normal direction and is called the SPOS face for contact definition. The 'bottom' surface is in the negative direction along the normal and is called SNEG face for contact definition [27]. The definition of SPOS and SNEG faces of membrane element is important not only during the contact definition, in which it is possible to define what face of the membrane is in contact with other auxiliary surfaces defined by rigid elements but also during the definition of the fluid cavity as described later.

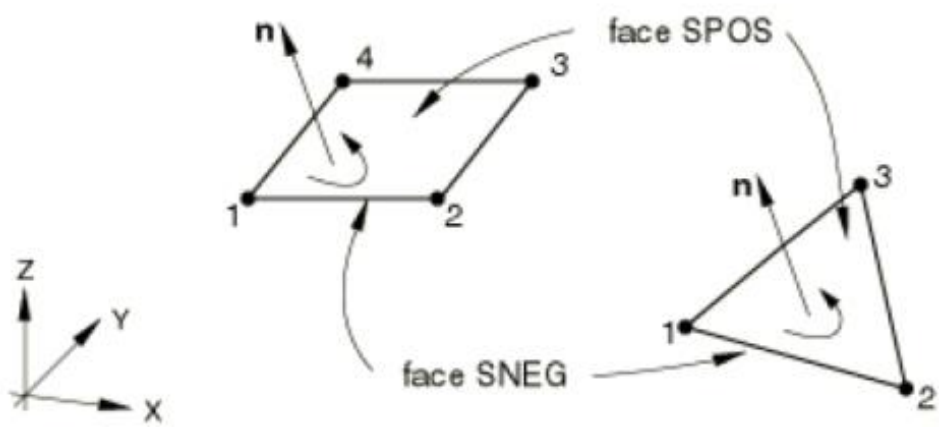

Figure 2.1. Normal orientation for membrane elements [27]. 


\subsubsection{Reference Mesh}

Similarly to the simulation of automobile airbags, the simulation of folding and deployment of large-scale inflatable structures requires the definition of a reference mesh (or initial metric file) that is used to restitute the membrane to the unstressed condition existing before the folding. In Abaqus, it is necessary to specify a reference mesh for membrane elements [27]. By calling the reference mesh, it is possible to restitute the inflatable structure to the desired final shape model and also to relieve the stresses that arose during the folding process. In this work, the reference mesh corresponded to the nominal shape of the inflatable before deflating and folding which was used as unstressed reference configuration.

In the simulation of deployment and inflation, two different meshes were defined in the input file (.inp): the folded flat shape and the reference mesh (contained in a .mtr file). This reference mesh contained the same element numbers and the coordinates of the nodes associated to the unstressed configuration of each element.

\subsubsection{Rigid Elements}

A rigid body or rigid element can be seen as a group of nodes, elements and/or surfaces whose motion is governed by the motion of single node called reference node. So, the motion of a rigid body can be defined by applying boundary condition at the reference node. During the simulation, the relative position of nodes and elements that are part of the rigid body remain constant. In this work, a rigid quadrilateral element named (R3D4) [27] was implemented for representing the auxiliary components such as the base, the folding planes, and the tunnel walls. In the R3D4 element, "R" stands for rigid element, "3D" represents the dimensionality of the element and " 4 " represent the number of nodes that define the element (quadrilateral element).

\subsubsection{Connector Elements}

With a connector element, it is possible to define a connection between two nodes and each node can be connected to a rigid part, a deformable part or not connected to any part. These elements are available for two dimensional, axis-symmetric, and three-dimensional analyses. The models created in this work included connectors of the type CONN3D2, where "CONN" stands for connector, "3D" stands for the dimensionality of the analysis and "N" represents the numbers of the nodes, which is always two because each connector is always defined by two nodes [27]. Once the type of connector is defined, it is important to define the type of connection and its behavior. There are several types of connections [27], but the models created in this work implemented only an axial connection which provided a connection between two nodes that acted along the connected line of the two nodes as shown in Figure 2.2. 


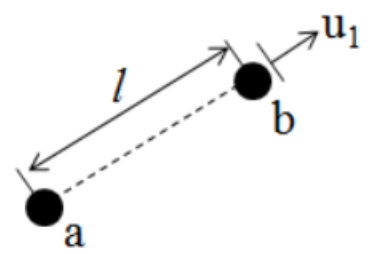

Figure 2.2. Connection type (axial) [27].

From Figure 2.2, the distance between the two nodes $a$ and $b$ is defined by:

$$
l=\left\|x_{b}-x_{a}\right\|
$$

The available displacement component $u_{1}$ of relative motion acts along the line connecting the two nodes is defined as:

$$
u_{1}=l-l_{0}
$$

Where $l_{0}$ is the initial distance from node $a$ and $b$.

The axial force is given by:

$$
f_{\text {axial }}=f_{1} \bar{q}
$$

Where:

$$
\bar{q}=\frac{1}{\| x_{b}-x_{a \|}}\left(x_{b}-x_{a}\right)
$$

is a measure of the nodes displacement with respect to its original position of the nodes.

Taking into account Eq. (2.8), the axial connection is defined by two parameters; the elasticity $\left(f_{1}\right)$ that defines its behavior; linear or non linear elastic effect on connector using elastic stiffness (force or moment per relative displacement or rotation) and $\bar{q}$. Moreover, a typical connector requires the definition of a failure behavior that represents the breaking of the connector when a relative motion component, force or moment becomes larger than a predefined reference value. If the failure criterion is met during the simulation, either all components or a single available component fail, the axial force $f_{\text {axial }}$ in each connector is removed during the increment when the failure criterion is met. 


\subsection{Material Properties}

The membrane of the inflatable structure was modeled as a single layer of fabric material. The fabric material model was considered orthotropic and non-linear. The constitutive model was defined by available experimental test data for each deformation direction. The response was represented by three components, two in tension in the warp and fill directions, and a third one to account for the shear response [27]. Although a fabric material does not have stiffness under compression, the stability and the convergence of the FE models required the definition of an artificial compressive strength in order to prevent excessive distortions or the collapse of membrane elements. For the models created in this work, a compressive strength in the range of $0.05 \%$ to $1 \%$ of the maximum tensile strength was assigned to the constitutive model of the fabric material.

\subsubsection{Material Damping}

From the equilibrium equation we know that:

$$
m \ddot{u}+c \dot{u}+k u=F
$$

The Rayleigh damping model introduces damping into the models in the following form [27]:

$$
c=\alpha m+\beta k
$$

where:

$\alpha$ is the mass proportional Rayleigh damping coefficient $\left[s^{-1}\right]$

$\beta$ is the stiffness proportional Rayleigh damping coefficient $\left[s^{-1}\right]$

$m$ is the mass matrix of the system

$k$ is the stiffness matrix of the system

If $\alpha=0$, the damping is proportional to the stiffness only, while if $\beta=0$ the damping is proportional to the mass only.

\subsection{Friction and Contact Properties}

During large displacement and deformation analyses, different parts of the inflatable structure can come into contact with itself and with other parts of the model. In order to avoid structural penetration, it is important to define contact interactions and contact controls [27, 29]. In this way, it is possible to represent 
the true physical events. In this work, the contact interactions were defined between the inflatable structure and the auxiliary components such as the base, the folding planes and the confined environment, and also between the inflatable structure and itself.

\subsubsection{Friction}

When two material bodies are in contact, the compressive force $\boldsymbol{F}_{\boldsymbol{N}}$ in the normal direction is built up as shown in Figure 2.3.

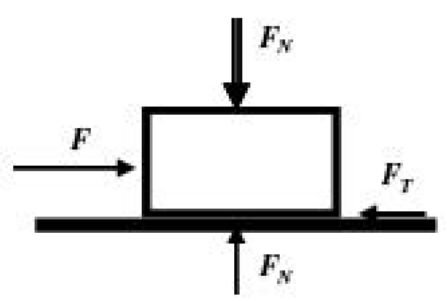

Figure 2.3. Friction between two material bodies in contact [29].

If the lateral force $\boldsymbol{F}$ tries to drive the upper body to slide away, there is a resistance force $\boldsymbol{F}_{\boldsymbol{T}}$ called friction force such that $\boldsymbol{F}_{\boldsymbol{T}}=-\boldsymbol{F}$, acting in the opposite direction of the driving force and it is tangent to the contact surface. When the driving force increases, the friction increases until it reaches its maximum value $\boldsymbol{F}_{\boldsymbol{T}_{\boldsymbol{m a x}}}$. Then, if $\boldsymbol{F}$ is smaller than $\boldsymbol{F}_{\boldsymbol{T}_{\text {max }}}\left(F<F_{T_{\max }}\right)$, the motion is not possible, but when $F>F_{T_{\max }}$, the sliding motion initiates.

There are two types of coefficients of friction, the static friction, and the dynamic friction. In this work, only the static friction was implemented in the models, and it is related to the friction between non-moving bodies, and it is defined by the ratio of tangential and normal force as:

$$
\mu_{s}=\frac{F_{T_{\max }}}{F_{N}}
$$

The friction in a mechanical system follows the Coulomb's model defined as:

$$
\begin{gathered}
\left|F_{T}\right| \leq \mu F_{N} \\
\left|F_{T}\right|<\mu F_{N} \Rightarrow v_{t}=0 \\
\left|F_{T}\right|<\mu F_{N} \Rightarrow \exists \lambda>0, v_{t}=-\lambda F_{T}
\end{gathered}
$$


Eq. (2.14) represents the situation of static friction. Eq. (2.15) states that friction force acts in the opposite direction of motion. Using mathematic notation, we can write:

$$
\frac{-F_{T}}{\left|F_{T}\right|}=\frac{-v_{T}}{\left|v_{T}\right|} \Rightarrow v_{T}=-F_{T} \frac{\left|v_{T}\right|}{\left|F_{T}\right|}=-\lambda F_{T}
$$

and, here $\lambda=\frac{\left|v_{T}\right|}{\left|F_{T}\right|}$ and $v_{T}$ is the tangential component of the velocity.

For the models created in this work, friction coefficients in the range of $0.1 \leq \mu \leq 0.5$ were implemented. Values close to the upper end of this range were adopted between the inflatable structure and the folding planes during the folding procedures in order to avoid sliding of material during the displacement of the folding planes.

During large deformation analyses, it is possible that the contact surfaces are involved in excessive penetrations. In most of the cases, as happened in this work, these excessive penetrations are due to the element size. The elements did not have enough space to deform and were involved in inter-element intersection and penetrations [28]. One way to avoid this problem was to use a more refined mesh in combination with the definition of a contact penalty factor which is described next.

\subsubsection{Contact Penalty Factor}

The purpose of the definition of a contact penalty factor is to detect and reduce the possibility of interelement intersections and penetration by using a force at the contact detection point(s) that has penetrated across the target surface. The mathematical formulation of this force is:

$$
f_{\text {contact }}=K_{c} h
$$

Where $K_{c}$ is the contact stiffness (also called contact penalty factor), and $h$ is the penetration size. From Eq. (2.17), it is possible to see that the larger the penetration, the greater will be the force $f_{\text {contact }}$. The challenge is to estimate the magnitude of this force. The force $f_{\text {contact }}$ needs to be large enough to push the contact surface back to the target surface in order to eliminate penetration but, at same time, not so large to push the contact out of the target surface. Therefore, the challenge is to choose an appropriate value of $K_{c}$. Several iterations were needed before finding the right value to eliminate the penetrations of elements at a reasonable computational cost. 


\subsection{Pressurization}

In this work, the simulation of pressurization of the inflatable followed two approaches. In the first approach, called "direct approach," the pressure was applied as a pneumatic pressure defined as a boundary condition to the surface of the membrane elements. In the second approach, called "indirect approach," the pressure applied on the surface of the membrane elements was derived from the definition on an inflator system that provided a specific gas mass flow rate for a specific period of time.

The main differences between the two approaches are the predictability of the pressure magnitude and its behavior. In the direct approach, the magnitude of the pressure and its behavior is directly defined in the model, whereas, in the indirect approach, only the mass flow rate is initially defined in the model, but the variation over the time of the magnitude of the internal pressure is part of the output of the simulation.

Both approaches required the definition of the coupling between the deformation of the inflatable structure and the internal pressure exerted by the gas on the structure. The deformation of the structure depended not only on the external pressure that acts on the model (gravity pressure) but also on the internal pressure exerted by the gas, which was assumed to be air. For modeling this coupling, a fluid cavity was defined to represent the volume being filled by the gas. The fluid cavity created for the models of this work was defined by the internal volume of the inflatable structure. The fluid cavity also required the definition of a cavity reference node that is associated with a fluid cavity. This reference node has only one degree of freedom (degree of freedom number 8) that was used to apply the internal pressure inside the fluid cavity [27].

\subsubsection{Direct Approach}

The direct approach was used during the controlled deflation carried out in preparation for modeling of the folding process. During the controlled deflations, the internal pressure was imposed as a boundary condition to the cavity reference node (degree of freedom number 8), and it was applied from the beginning of the simulation with a magnitude equal to the external pressure (gravity pressure), and then gradually decreased during the simulation. In this approach, at the beginning of the simulation, there was an equilibrium between internal pressure and external pressure. During the simulation of controlled deflation, the pressure decreased with a ramp function, and the inflatable structure started to deflate due to the unbalance between internal and external pressure until it reached the flat shape. That is, at the beginning of the simulation:

$$
P_{\text {internal }}=P_{\text {external }}
$$


Then, during the simulation:

$$
P_{\text {internal }}<P_{\text {external }}
$$

And, at the end of simulation:

$$
P_{\text {internal }}=0
$$

\subsubsection{Indirect Approach}

The indirect approach was used to simulate the inflation process under unconfined and confined conditions. During the inflation, the pressure applied inside of a cavity was achieved by modeling the transfer of fluid into the cavity. The fluid transferred to the cavity was assumed to be air with the behavior of an ideal gas. The flow of air was specified as a prescribed mass flow rate history and also by defining a fluid exchange between the inflator and the fluid cavity.

A specified air mass flow rate filled the internal volume equal to the nominal volume corresponding to the nominal geometry of the inflatable structure, plus an additional volume $V_{m}$ resulting from the stretch of the membrane produced by the internal pressure. The mass flow rate is linked to the other thermodynamic variables by the ideal gas equation:

$$
P V=m R T
$$

where $P, V, m, T$ and $R$ are the internal absolute pressure in the inflatable, the inflatable target internal volume, the mass of the gas introduced by the inflator, the internal gas temperature and the universal gas constant, respectively.

The inflator implemented in this work to fill the cavity follows the model proposed by Wang [16] and implemented in Abaqus Explicit [27]. In this model, the rate of the air mass introduced into the control volume is given by:

$$
\frac{d m}{d t}=\dot{m}
$$

Where, $\dot{m}$ is the rate of air mass that flows from the inflator into the cavity. The thermodynamic properties of the air that fills the cavity are determined starting from the conservation of energy. The inflation is assumed to follow an adiabatic transformation in which the process occurs without transfer of heat or matter 
between the thermodynamic system and its surroundings $[16,30]$. Under this condition, the change of heat can be written as:

$$
\Delta Q(t)=0
$$

Also, the conservation of energy is given by:

$$
\frac{d}{d t}\left(m c_{v} T\right)=c_{p} T \dot{m}
$$

Where $c_{v}$ and $c_{p}$ are the specific heat of the gas at constant volume and pressure, respectively. Rewriting Eq. (2.21), we get:

$$
T=\frac{P V}{m R}
$$

Substituting Eq. (2.25) on the left-hand side of equation Eq. (2.2) results:

$$
\dot{m}=\frac{\dot{P} V}{R k T}
$$

where $R=\left(c_{p}-c_{v}\right)$ and $k=\frac{c_{p}}{c_{v}}$.

Since the inflator gas temperature has a very small influence on the final value of pressure, it is considered constant and equal to the value of environment temperature [16]. This consideration makes the inflation process a nearly isothermal process. An isothermal process usually happens when the process is relatively slow, as in the inflation simulations developed in this work.

In Eq. (2.26), $\dot{P}$ represents the variation over the time of absolute pressure. In order to estimate the absolute pressure of a system $P_{0}$, the atmospheric pressure $P_{a}$ must be added to the gauge pressure $P_{g}$ (internal pressure measurement):

$$
P_{0}=P_{g}+P_{a}
$$

Abaqus/Explicit requires the definition of the heat capacity at constant pressure to model an adiabatic process [31]. The heat capacity at constant pressure was defined using a polynomial form based on the Shomate equation: 


$$
\tilde{c}_{p}=\tilde{a}+\tilde{b}\left(\theta-\theta^{z}\right)+\tilde{c}\left(\theta-\theta^{z}\right)^{2}+\tilde{d}\left(\theta-\theta^{z}\right)^{3}+\frac{\tilde{e}}{\left(\theta-\theta^{z}\right)^{2}}
$$

Where, $\tilde{a}, \tilde{b}, \tilde{c}, \tilde{d}$ and $\tilde{e}$ are gas constants and $\theta$ is the current temperature and $\theta^{z}$ is absolute zero temperature on the unit system adopted. The constant pressure heat capacity can be obtained by the following relation:

$$
c_{p}=\frac{\tilde{c}_{p}}{M W}
$$

Where $M W$ is the molecular weight of the gas. 


\section{Chapter 3. Model Generation}

\subsection{Introduction}

This chapter describes all the geometries and dimensions of all the parts modeled in this work including the inflatable structure, the tunnel section, and the folding planes. This chapter also describes details regarding the generation of FE models including material properties, mesh convergence analysis for the model corresponding to the inflatable structure.

\subsection{Nominal Geometries and Dimensions}

The units adopted in this work correspond to the International System of Units (SI), more precisely the MKS system of units in which distance are measured in meters $(\mathrm{m})$, mass in kilograms $(\mathrm{kg})$ and time in seconds $(s)$.

The two main components of the model are the inflatable structure and the tunnel segment. The Inflatable structure modeled in this work consists of a cylinder with two spherical end caps. The radius of the cylinder is $2.794 \mathrm{~m}$ and its length is $3.657 \mathrm{~m}$ and the radii of the spherical end caps are $3.658 \mathrm{~m}$ [7]. Figure 3.1 shows the overall shape and dimensions of the inflatable structure used in the analysis presented in the following sections. Figure 3.2 shows the cross-section of the tunnel segment in which the folded inflatable structure will be positioned and inflated.

As seen in Figure 3.2, the nominal diameter of the tunnel is $5.020 \mathrm{~m}$. The folded inflatable structure will be attached and stored on the ceiling of the tunnel (segment $A B$ ). The maximum width of the folded inflatable structure cannot exceed a width of $1.450 \mathrm{~m}$ in order to fit in the available room. According to the dimensions of the inflatable structure shown in Figure 3.1 and the dimensions of the tunnel shown in Figure 3.2 , the perimeter of the cylindrical part of the inflatable structure is $17.550 \mathrm{~m}$ and the perimeter of the cross section of the tunnel is $5.810 \mathrm{~m}$. With these dimensions, the cylindrical perimeter of the inflatable is $11 \%$ larger than the perimeter of the tunnel. This increase of the perimeter of the inflatable is to account for the possibility of bridging around the corners and the presence of other elements that could interfere with the local conformity of the inflatable to the tunnel perimeter [7].

During the deflation and folding process, the inflatable structure will interact with a flat surface called "base" which is representative of the floor where the folding procedures take place. Moreover, rigid folding planes are also used to simulate the folding procedures implemented experimentally [7]. The base and the folding planes are shown in Figure 3.3. 


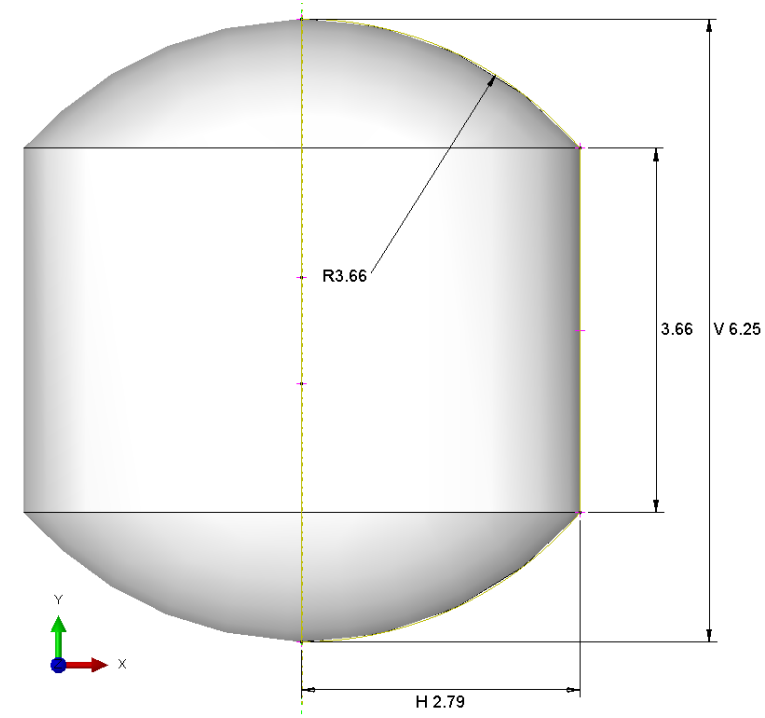

Figure 3.1. Inflatable structure geometry and dimensions (dimensions in meters).

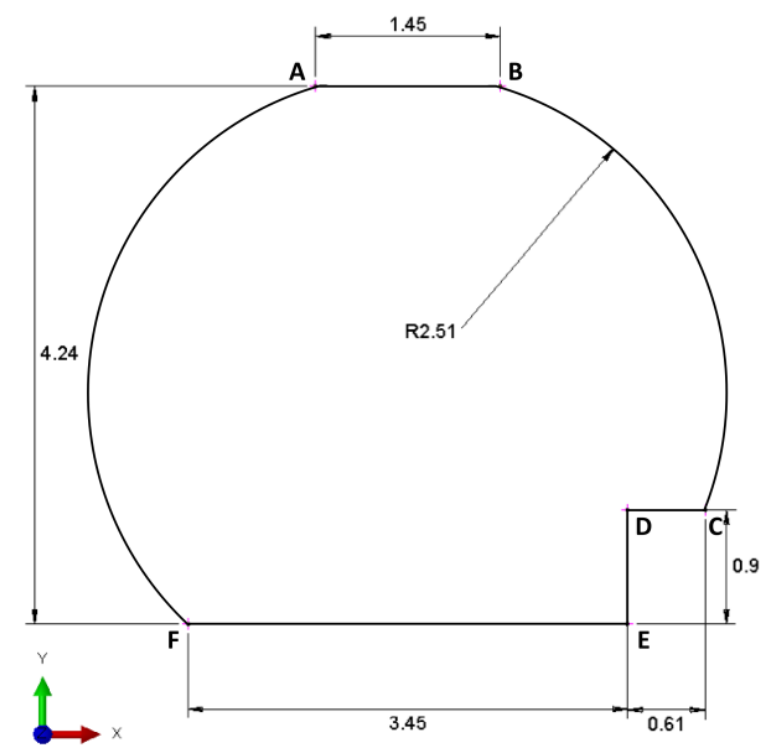

Figure 3.2. Tunnel cross-section dimensions (dimensions in meters).

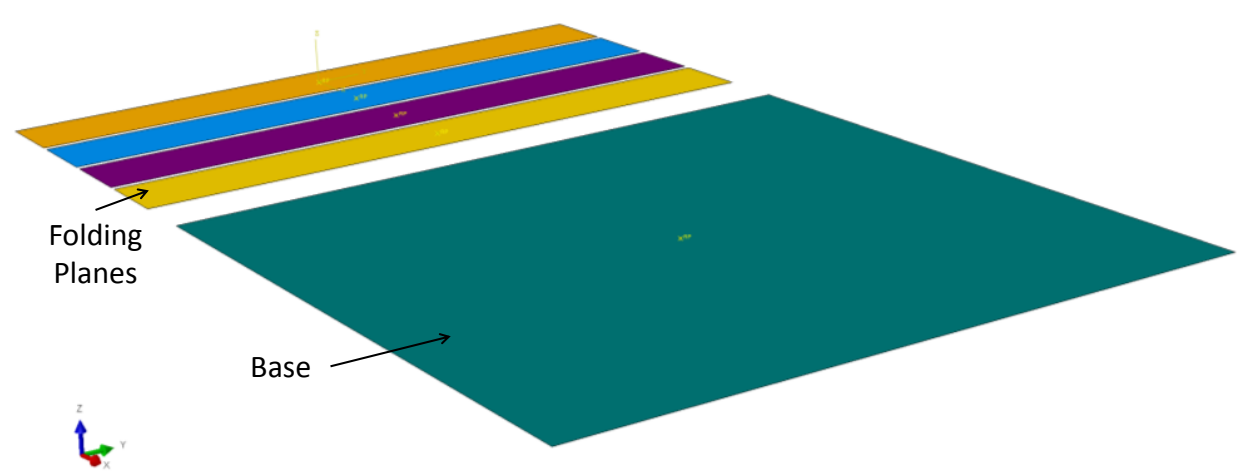

Figure 3.3. Base and folding planes. 
The base is represented by a square surface of 10 by 10 meters, and rectangles represent the folding planes. In the preliminary stages of the model creation, the four folding planes had the same size (length of $10 \mathrm{~m}$ and width of $1.25 \mathrm{~m}$ ). Since the final configuration of the flat shape used during the folding process is unknown, the dimensions of the planes will be then modified (by adjusting the nodal coordinates) during the simulation process according to the folding sequence being modeled.

\subsection{Generation of FE models}

\subsubsection{Inflatable Structure}

The generation of the FE model of the inflatable structure was completed during the pre-processing in which the geometry of the model, material properties, element type and other properties were defined. The initial geometry of inflatable structure was created using three-dimensional deformable shell through Abaqus/CAE. The shell surface was then partitioned in several auxiliary surfaces as shown in Figure 3.4.

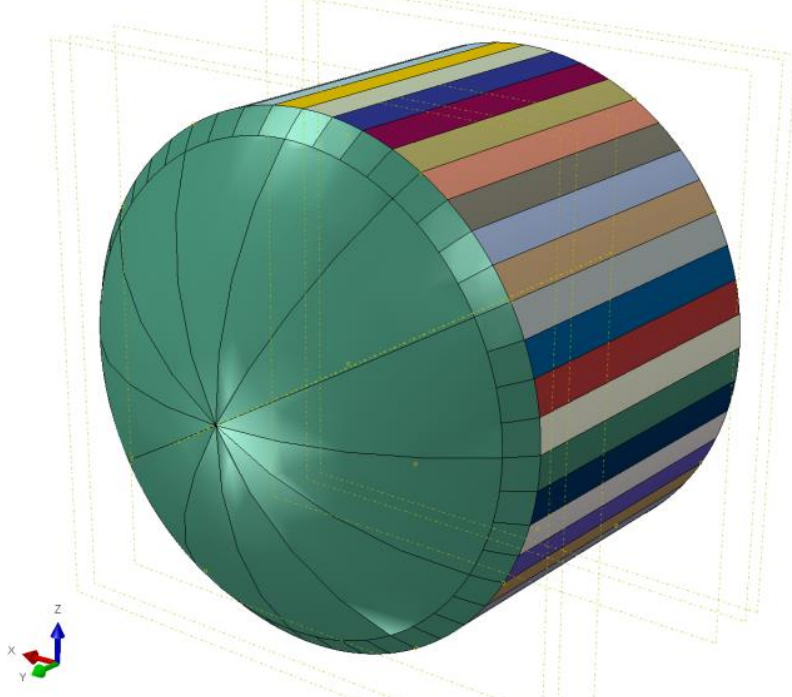

Figure 3.4. Inflatable structure, FE initial geometry, and partitions generated using Abaqus/CAE.

The partitions on the cylindrical part of the inflatable structure were created in order to define folding surfaces and folding lines that were very useful as reference lines at the different stages of the simulations. Additional surface partitions were created on the spherical end caps in order to have a more uniform mesh. The membrane of the inflatable structure is a single layer coated fabric with a thickness of $t=0.00078 \mathrm{~m}$ and density of $\rho=1346 \frac{\mathrm{Kg}}{\mathrm{m}^{3}}$. The models built in this work implement the mechanical properties of Ferrari Precontraint $1002[32,34]$. The material is assumed to behave as an orthotropic fabric with tensile strengths in the warp and fill directions. The fabric also includes shear strength adapted from the experimental results 
reported in [33]. The mechanical behavior under tensile loads of the material is shown in Figure 3.5(a) and the mechanical behavior under shear loads is shown in Figure 3.5(b).

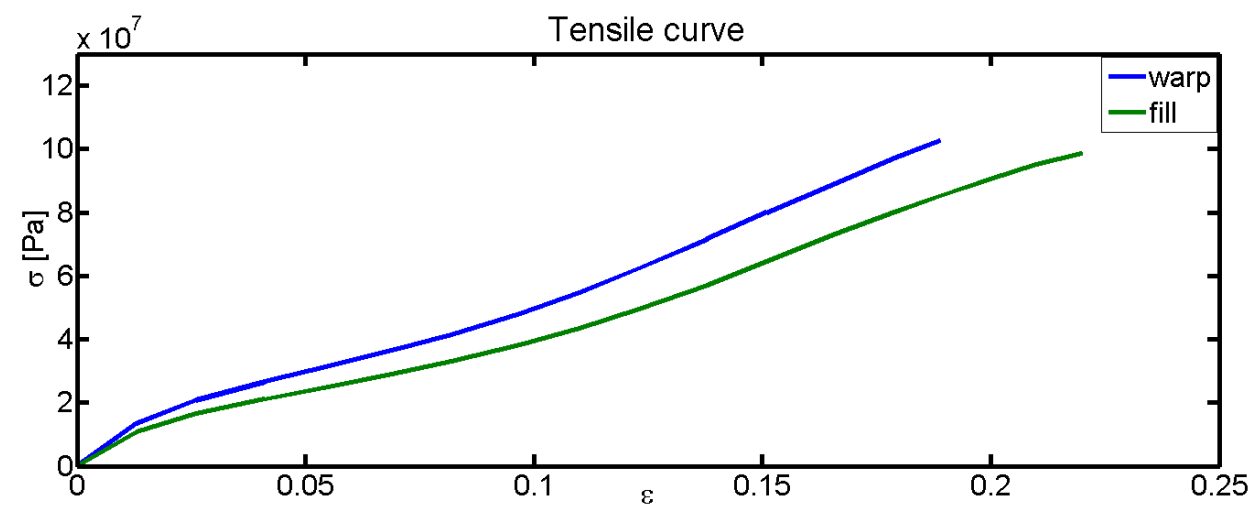

(a)

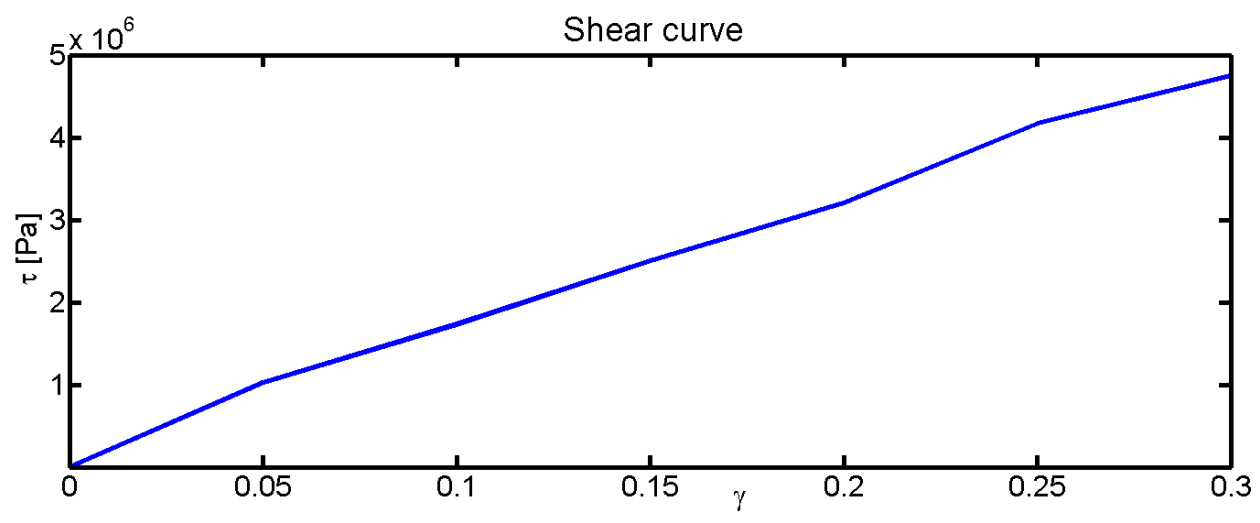

(b)

Figure 3.5. Constitutive model of fabric material: (a) Mechanical behavior under tensile load; (b) Mechanical behavior under shear loads.

Although the actual fabric material does not have stiffness under compression, the stability and the convergence of the FE models required the definition of an artificial compressive strength in order to prevent excessive distortions or the collapse of membrane elements. The inclusion of a small compressive strength is common in the simulation of automobile airbags [27]. For the models created in this work, different compressive strengths in the range of $0.05 \%$ to $1 \%$ of the maximum tensile strength were assigned to the constitutive model according to the type of simulation process being performed: deflation, folding or inflation. 


\subsubsection{Stress Evaluation and Mesh Convergence Study}

The membrane of the inflatable was modeled using M3D3 membrane elements [27]. The properties of the M3D3 element were described in Section 2.3.1. Three different mesh densities were generated to evaluate the membrane stresses of the inflatable structure under unconfined pressurization conditions. The nominal shape illustrated in Figure 3.4 is modeled with 27528, 48948 and 95902 elements and these meshes were identified as Mesh A, Mesh B, and Mesh C, respectively. The inflatable structure was pressurized in an unconfined condition using an internal pressure of $P=6.89 \cdot 10^{3} \mathrm{~Pa}$ (or $1 \mathrm{psi}$ ) which corresponds to the value of the internal (or gauge) pressure measured during the experiments reported in [7] at the end of the inflation process.

The circumferential or hoop stress $\left(\sigma_{1}\right)$ and the longitudinal stress $\left(\sigma_{2}\right)$ on the cylindrical portion and on the spherical end caps were evaluated first analytically and then numerically in order to estimate the level accuracy of the FE models with different mesh densities.

The analytical values corresponding to $\left(\sigma_{1}\right)$ and $\left(\sigma_{2}\right)$ for cylindrical and spherical end caps were evaluated using classical equations for thin-walled structures under internal pressure as follows:

$$
\begin{gathered}
\sigma_{1_{\text {Cylinder }}}=\frac{P R_{\text {Cylinder }}}{t}=24.75 \cdot 10^{6} \mathrm{~Pa} \\
\sigma_{2_{\text {Cylinder }}}=\frac{P R_{\text {Cylinder }}}{2 t}=12.38 \cdot 10^{6} \mathrm{~Pa} \\
\sigma_{1_{\text {cap }}}=\sigma_{2_{\text {cap }}}=\sigma=\frac{P R_{\text {cap }}}{2 t}=16.18 \cdot 10^{6} \mathrm{~Pa}
\end{gathered}
$$

where $P$ is the internal pressure equal to $6.89 \cdot 10^{3} \mathrm{~Pa} t$ is the membrane thickness, $R_{C y l i n d e r}$ is the radius of the cylindrical region equal to $2.795 \mathrm{~m}$ and $R_{c a p}$ is the radius of the spherical end cap equal to $3.658 \mathrm{~m}$. For the evaluation of the mesh convergence, the hoop stress (S11) in the cylindrical region was chosen as a control parameter. The stress distribution contours in hoop directions related to the three mesh densities are shown in Figure 3.6. 

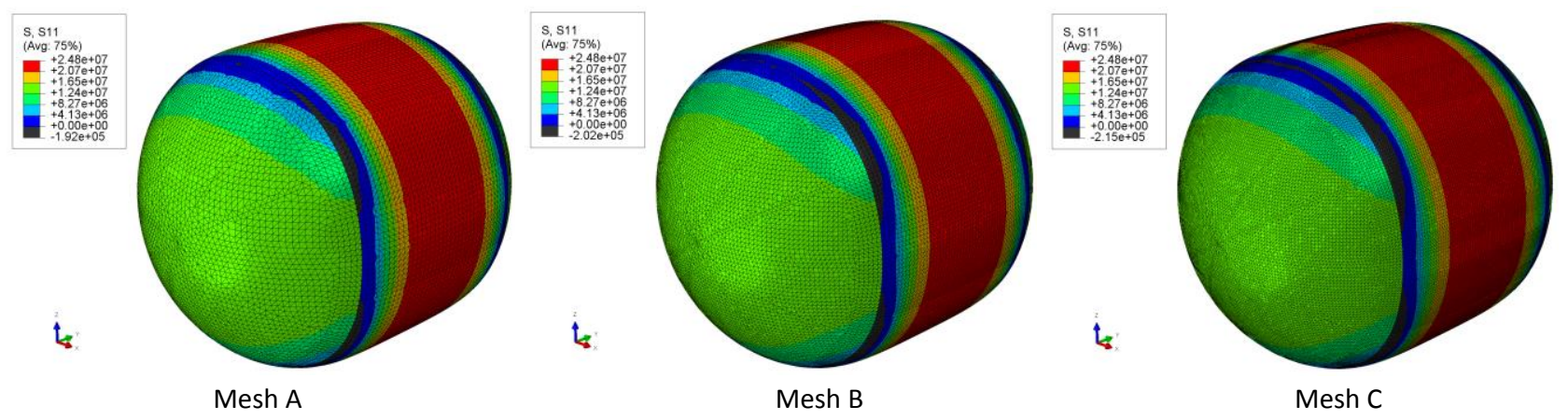

Figure 3.6. Stress distribution contour for Mesh A, Mesh B, and Mesh C.

Four nodes around the middle cross section of the cylinder region were chosen to evaluate the average of the hoop stresses as shown in Figure 3.6. Table 3.1 summarizes the FE results and compares them with the analytical solution.

Table 3.1. Summary of mesh convergence study.

\begin{tabular}{|l|c|c|c|}
\hline \multirow{2}{*}{} & Element Size & \multicolumn{2}{|c|}{ Cylindrical Region } \\
\cline { 2 - 4 } & {$[\mathrm{m}]$} & $S_{11}[\mathrm{~Pa}]$ & $\%$ Error \\
\hline Analytical value $\sigma_{1}$ & & $24.75 \cdot 10^{6}$ & \\
\hline Mesh A & 0.07 & $24.70 \cdot 10^{6}$ & 0.21 \\
\hline Mesh B & 0.06 & $24.68 \cdot 10^{6}$ & 0.29 \\
\hline Mesh C & 0.05 & $24.70 \cdot 10^{6}$ & 0.22 \\
\hline
\end{tabular}

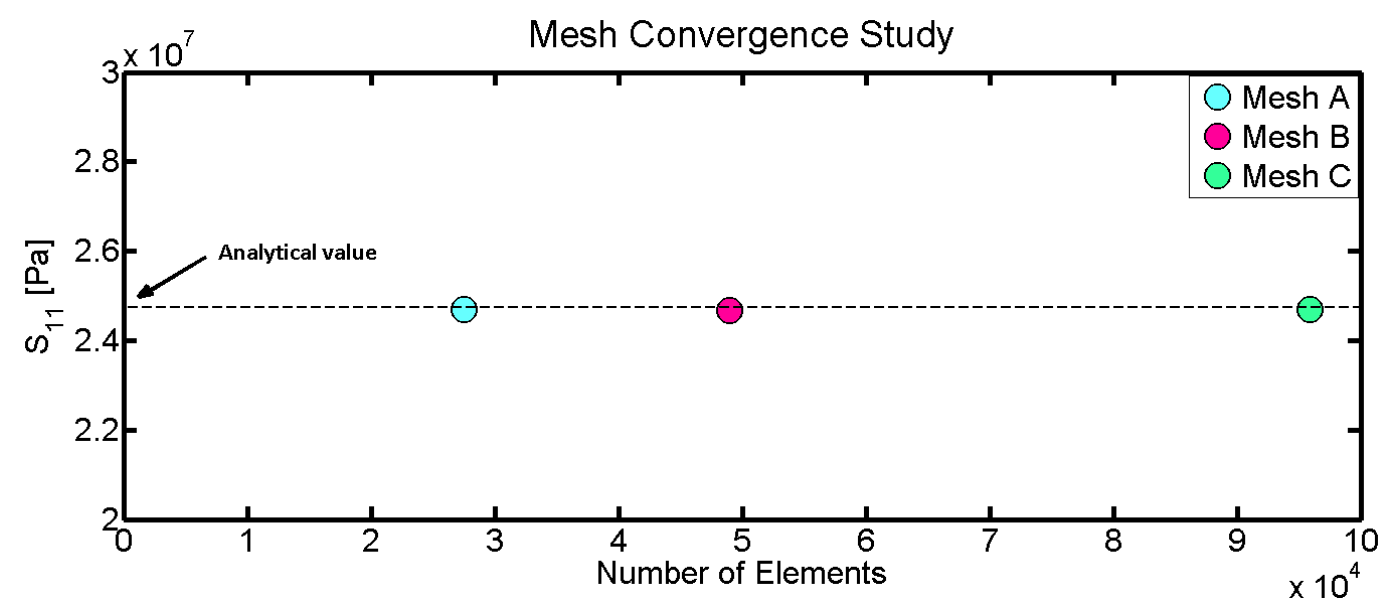

Figure 3.7. Mesh convergence.

Table 3.1 and Figure 3.7 show that the difference between the values of the analytical solution and the values of the FE simulations is negligible indicating that any of the proposed meshes would predict the 
stress with a reasonable accuracy. Although it is recognized that a more dense mesh is more expensive in terms of computational time, the implementation of folding procedures will require a relatively refined mesh in order minimize the volume of the final folded shape and the same time prevent inter-element penetrations and intersections. For these reasons, Mesh $\mathrm{C}$ will be adopted in the remainder models described in the following chapters.

\subsubsection{Tunnel, Base, and Folding Plates}

The FE model of the tunnel, base and folding planes were created via three-dimensional rigid shell surfaces generated in Abaqus/CAE. Since these surfaces are considered non-deformable, they were meshed using linear quadrilateral rigid elements R3D4. The properties of this type of element were described in Section 2.3.3.

The mesh of the tunnel segment is comprised of 4914 elements with a size of $0.20 \mathrm{~m}$. The mesh of the base is formed by 400 elements with size of $0.5 \mathrm{~m}$, whereas the mesh of the folding planes is formed by 40 elements. The meshes of the tunnel, base and folding planes are shown in Figure 3.8.

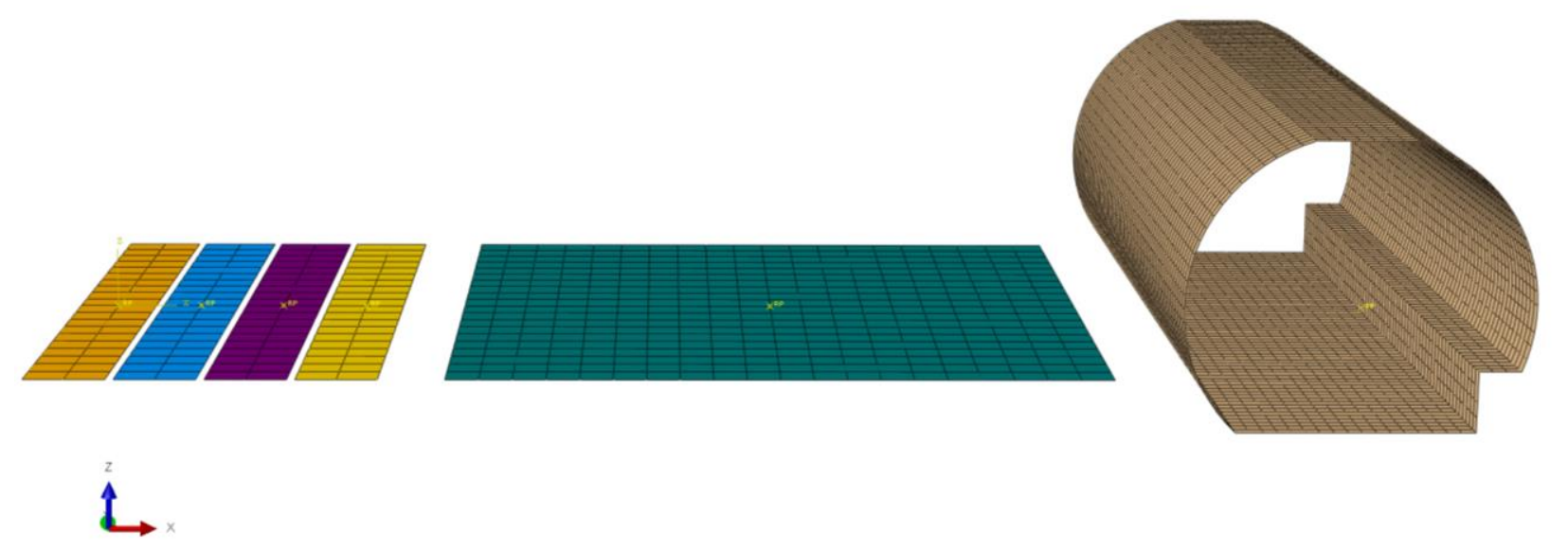

Figure 3.8. Meshes of folding planes, base and tunnel segment.

After the creation of the different components of the FE model, all the nodes and elements of meshed geometries were renumbered with HyperMesh [28] and prepared to be utilized with Abaqus Scripting in order to make the simulation work more efficient. Furthermore, a reference mesh or (initial metric) of the nominal shape of the inflatable structure was created using HyperMesh in which all the element numbers and the coordinates of all the element's nodes were specified. The purpose of the reference mesh was detailed in Section 2.3.2. 


\subsection{Summary}

This chapter presented the geometric properties, materials, and meshes of all the parts implemented in this work. The results of the mesh convergence study for the inflatable structure showed that even when a relatively coarse mesh can predict the stresses with a small margin of error, a model with a more dense mesh was selected to reproduce the folding procedures better and also minimize the volume of the final folded shape and, at the same time, reduce the possible occurrence of inter-element penetrations and intersections. 


\section{Chapter 4. Deflation and Unconfined Inflation}

\subsection{Introduction}

Starting from the nominal shape of the inflatable structure described in the previous chapter, two techniques are introduced in this chapter: the uncontrolled deflation and controlled deflation. The details of these two techniques are discussed highlighting the differences and the improvements in the final deflated shape. This chapter also presents the properties of the inflator implemented in the simulation of an unconfined inflation process. The unconfined inflation process is used to test the inflator definition and to verify if the target values of volume and pressure are achieved. The chapter ends with a parametric study performed to assess how the changes of different parameters influence the behavior of the membrane material as well the computational time.

\subsection{Deflation}

The main purpose of the simulation of deflation was to reach the flattest possible shape with the minimum amount of wrinkles on the flattened membrane in order to minimize the volume of the final folded shape. Two approaches were adopted to achieve a flat shape: 1) by performing an uncontrolled deflation and, 2) by performing a controlled deflation. In both approaches, an internal decreasing uniform pressure was applied using the direct approach described in Section 2.6.1. The application of an internal uniform pressure was essential in order to prevent the immediate collapse of the membrane due to the application of the gravity load. The main difference between the two approaches was on the number of steps in which they were performed and on the slope adopted for the ramp function used to simulate the decreasing of the internal pressure.

\subsubsection{Uncontrolled Deflation}

In this approach, the simulation started with the nominal shape of the inflatable structure subjected to an internal pressure equal to the gravity pressure to balance the external load due to the application of gravity. The evaluation of the gravity pressure is shown in the following procedure:

$$
\begin{gathered}
P_{\text {gravity }}=\frac{F_{(\text {gravity })}}{S_{(\text {inflatable })}}=\frac{m_{(\text {inflatable })} g}{S_{(\text {inflatable })}}=\frac{\rho V_{(\text {inflatable })} g}{S_{(\text {inflatable })}} \\
V_{(\text {inflatable })}=V_{(\text {cylinder })}+V_{(\text {end caps })}=0.0967 \mathrm{~m}^{3}
\end{gathered}
$$




$$
S_{(\text {inflatable })}=S_{(\text {cylinder })}+S_{(\text {end caps })}=123.867 \mathrm{~m}^{2}
$$

Where $\rho$ is the density of the fabric material equals to $1346 \frac{\mathrm{Kg}}{\mathrm{m}^{3}}$ and $g$ is the acceleration of gravity equals to $9.81 \frac{\mathrm{m}}{\mathrm{sec}^{2}}$. The product $\left(\rho V_{\text {(inflatable })}\right)$ gives the total mass of the inflatable equals to $130 \mathrm{~kg}$.

Substituting Eq. (4.2) and Eq. (4.3) into Eq. (4.1), we get:

$$
P_{\text {gravity }}=\frac{130 \times 9.81}{123.867}=10.3 \mathrm{~Pa}
$$

During the uncontrolled deflation, an internal pressure $\left(P_{\text {internal }}\right)$ equal to the gravity pressure ( $\left.P_{\text {gravity }}\right)$ was applied to the nominal shape of the inflatable while standing on the base. At the beginning of the simulation (time step $\mathrm{t}=0)$ the internal and external pressure $\left(P_{\text {internal }}=P_{\text {gravity }}\right)$ were in equilibrium. Then during the simulation, the amplitude of the internal pressure was reduced using a ramp function with a slope of $0.01 \mathrm{~Pa} / \mathrm{sec}$. The unbalance between internal and external pressures produced the deflation of the inflatable $\left(P_{\text {internal }}<P_{\text {gravity }}\right)$ until it reached a relatively flat shape when the internal pressure was equal to zero $\left(P_{\text {internal }}=0\right)$ at the end of simulation. The sequence of the uncontrolled deflation is illustrated in Figure 4.1. Additional estimated parameters implemented in this technique included: a) a compressive strength equal to $0.05 \%$ of the tensile strength; b) a mass scaling factor (MSF) of 10 was included to reduce the simulation time; and, c) a mass proportional damping factor $\alpha=0.0$ (no damping).

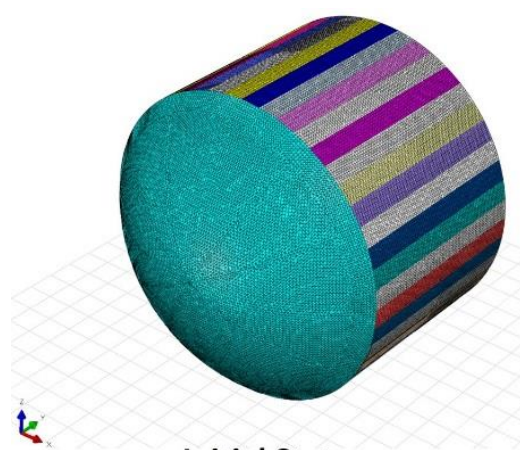

Initial State

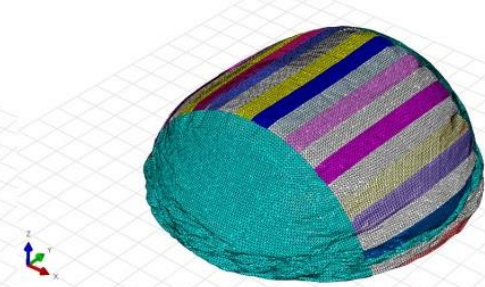

Intermediate State

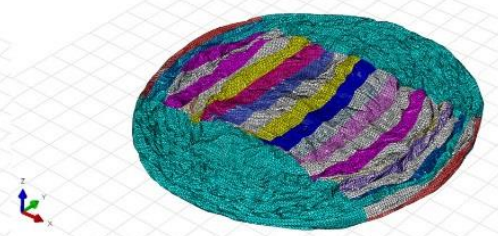

Final State

Figure 4.1. Sequence of uncontrolled deflation.

As seen from the sequence of images of Figure 4.1, although the internal pressure was able to prevent the immediate collapse of the membrane at the beginning of the simulation, the reduction of the pressure was so fast that it was not able to prevent the collapse of the membrane until the end of the simulation. The rapid collapse of the membrane material would explain the relatively large wrinkles around the border of the flat shape illustrated in Figure 4.1 (Final State). 


\subsubsection{Controlled Deflation}

The controlled deflation was created as an improvement of the previous technique. Since the development of large wrinkles depends on how fast the internal pressure is reduced, the controlled deflation was performed using a slope for the ramp function equal to $0.25 \%$ of the value used in the uncontrolled deflation. Although the controlled deflation was performed in one step as done in the previous technique, the simulation was stopped every 3 to 4 iterations in order to have a better control of the collapse of the membrane. After each interruption, the coordinates of the resultant shape were exported first to Abaqus/CAE and then to Hypermesh in order to inspect the mesh and detect if the membrane elements were affected by inter-element penetrations and intersections, and in such case, correct them before preceding the simulation once again. After each verification step, the controlled deflation with the corrected mesh continued using the same initial conditions of pressure $\left(P_{\text {internal }}=P_{\text {gravity }}\right)$ and continued with the same decreasing ramp. This process was repeated nineteen times (19 steps), and each step was denominated cleaning process. Each cleaning step contributed to remove all the larger and most of the smaller wrinkles seen in the uncontrolled deflation. Using this approach, it was possible to achieve a better control of the membrane material which allowed a better distribution of the membrane by eliminating major wrinkles, located mainly around the border of the flattened shape. Additional node displacement were applied on the bottom part of the inflatable structure in order to achieve a flatter shape in the region of the inflatable that will be then attached to the ceiling of the tunnel. A series of images showing the sequence of the controlled deflation is shown in Figure 4.2.

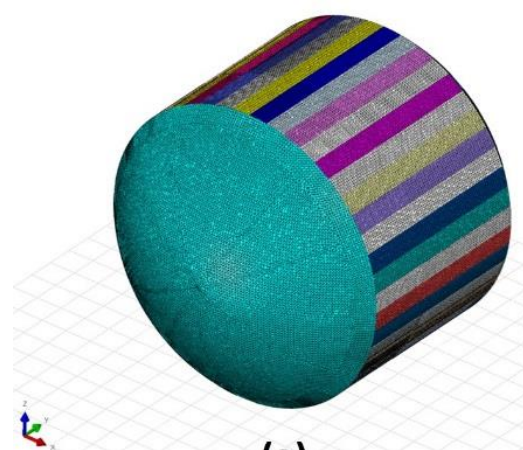

(a)

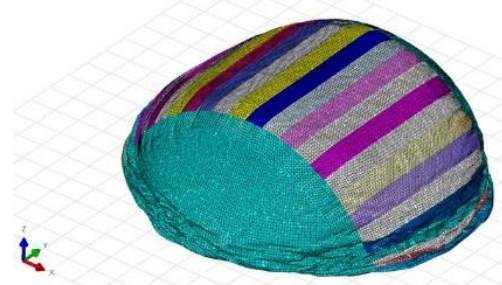

(d)

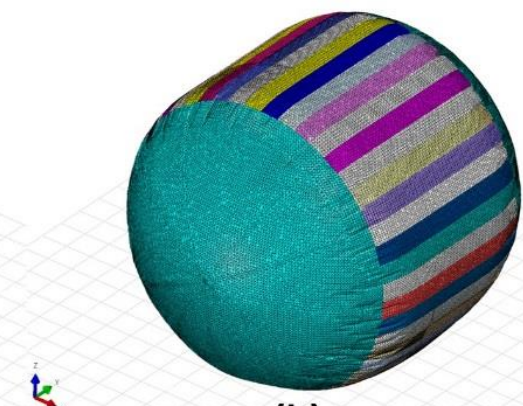

(b)

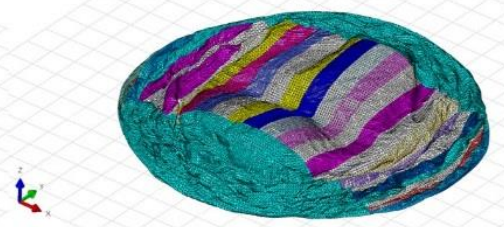

(e)

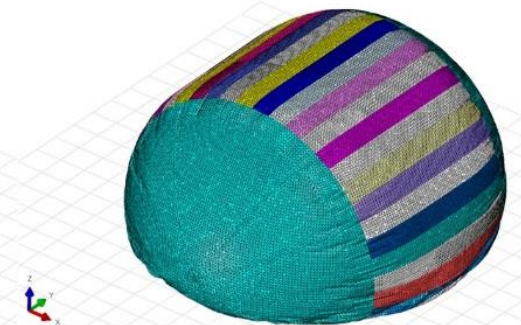

(c)

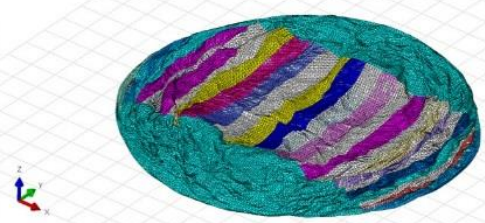

(f)

Figure 4.2. Sequence of controlled deflation. 
Since the membrane of the inflatable structure was very light due to the thickness and density of the fabric material, and in order to reach a flatter shape, an additional load equal to 10 times the gravity load was applied as a uniform load onto the shape as shown in Figure 4.2(f). The final shape after completion of the controlled deflation and application of additional external load is shown in Figure 4.3.

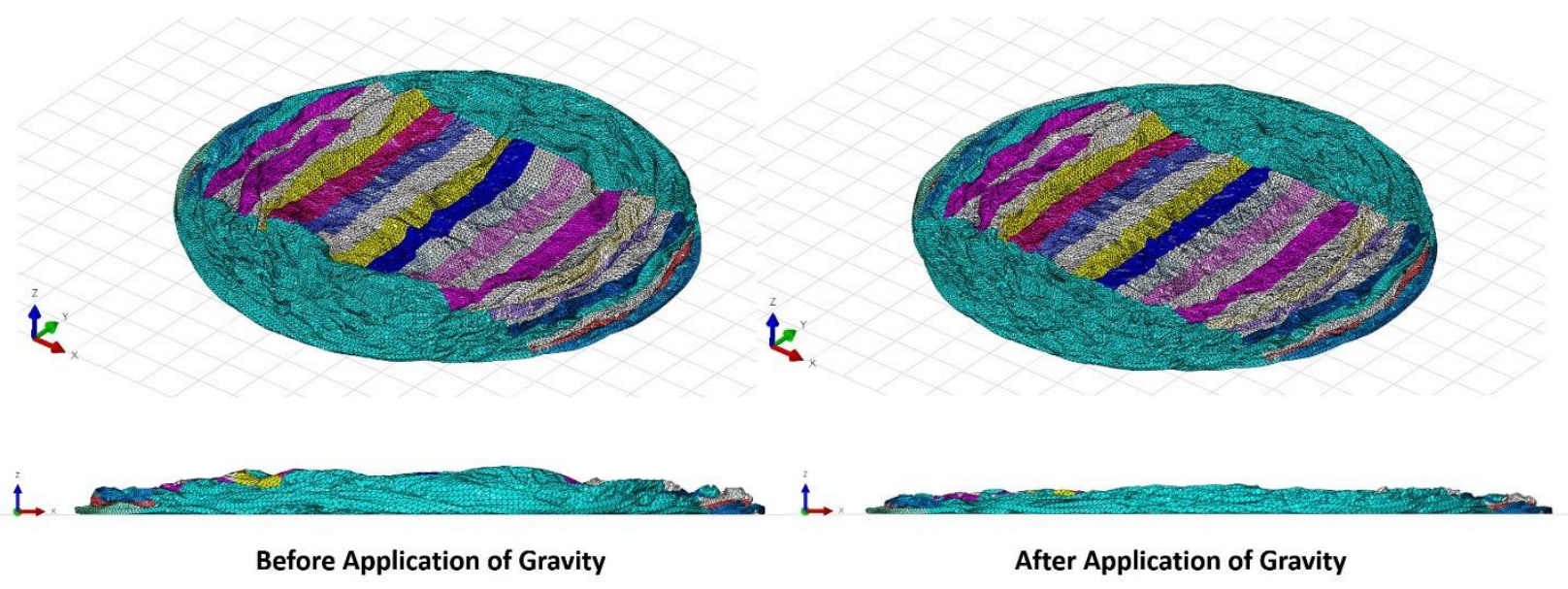

Figure 4.3. Flat shape after controlled deflation and application of gravity load.

\subsection{Inflator Design for Unconfined Inflation}

In Abaqus/Explicit, the definition of an inflator requires the definition of a gas mass flow rate and a gas temperature as a function of the inflation time. The gas used to fill the volume of the inflatable structure was air. During the unconfined inflation, the temperature of the gas was kept constant and equal to the ambient temperature. The properties of air needed for the definition of the inflator are summarized in Table 4.1 and Table 4.2:

Table 4.1. Thermodynamic properties of Air [35].

\begin{tabular}{|l|l|c|}
\hline \multicolumn{3}{|c|}{ Thermodynamic properties of Air } \\
\hline Universal Gas Constant & 8314.3 & $\mathrm{~J} /(\mathrm{kmol} \cdot \mathrm{K})$ \\
\hline Molecular Weight & 28.97 & $\mathrm{~kg} / \mathrm{kmol}$ \\
\hline Absolute Temperature & 0.0 & $\mathrm{~K}$ \\
\hline Ambient Temperature at sea level & 288.15 & $\mathrm{~K}$ \\
\hline Ambient Pressure at sea level & 101315.0 & $\mathrm{~Pa}$ \\
\hline Density of Air & 1.225 & $\mathrm{~kg} / \mathrm{m}^{3}$ \\
\hline$\gamma$ & 1.4 & \\
\hline
\end{tabular}


Table 4.2. Coefficient of Shomate equation [31].

\begin{tabular}{|c|c|c|}
\hline \multicolumn{3}{|c|}{ Coefficients of Shomate Equation } \\
\hline $\mathrm{a}$ & 28110 & $\mathrm{~J} / \mathrm{kmol} \cdot \mathrm{K}$ \\
\hline $\mathrm{b}$ & 1.967 & $\mathrm{~J} / \mathrm{kmol} \cdot \mathrm{K}^{2}$ \\
\hline $\mathrm{c}$ & 0.004802 & $\mathrm{~J} / \mathrm{kmol} \cdot \mathrm{K}^{3}$ \\
\hline $\mathrm{d}$ & -0.000001966 & $\mathrm{~J} / \mathrm{kmol} \cdot \mathrm{K}^{4}$ \\
\hline $\mathrm{e}$ & 0.0 & $\mathrm{JK} / \mathrm{kmol}$ \\
\hline
\end{tabular}

The air mass flow rate for an unconfined inflation was designed to fill the total volume of the inflatable in 200 seconds. The total volume adopted for definition of the inflator included the nominal volume of the inflatable plus an increment of volume due to the stretch of the membrane produced by the internal target pressure of $P=6,89 \cdot 10^{3} \mathrm{~Pa}$ (or $1 \mathrm{psi}$ ) expected to be reached at the end of the inflation [7].

\subsubsection{Evaluation Total Inflation Volume}

From the nominal geometry shown in Figure 3.4, the nominal volume is $V_{0}=123.27 \mathrm{~m}^{3}$. The problem now is to evaluate how much the volume can expand due to an internal pressure $P$. The stresses $\sigma_{1}$ and $\sigma_{2}$ were evaluated on the cylindrical and spherical end caps using the classical equations for thin-walled structures under internal pressure $P$ as shown in Eq.(3.1), Eq.(3.2) and Eq.(3.3). From the constitutive model shown in Figure 3.5, it can be seen that the stresses calculated for a pressure $P$ were in the linear range of the stress-strain relationship. Then, it is possible to use the following proportions to evaluate $\varepsilon_{1_{\text {cylinder }},} \varepsilon_{2_{\text {cylinder }}}$ and $\varepsilon_{\text {caps }}$ :

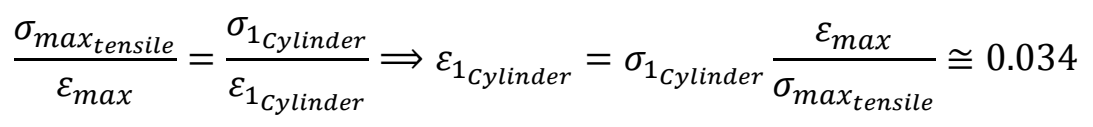

$$
\begin{aligned}
& \frac{\sigma_{\text {max }} \text { tensile }}{\varepsilon_{\text {max }}}=\frac{\sigma_{2_{\text {Cylinder }}}}{\varepsilon_{2_{\text {Cylinder }}}} \Rightarrow \varepsilon_{2_{\text {Cylinder }}}=\sigma_{2_{\text {Cylinder }}} \frac{\varepsilon_{\text {max }}}{\sigma_{\text {max }}} \underset{\text { tensile }}{ } \cong 0.017 \\
& \frac{\sigma_{\text {max }} \text { tensile }}{\varepsilon_{\max }}=\frac{\sigma_{\text {cap }}}{\varepsilon_{\text {cap }}} \Rightarrow \varepsilon_{\text {cap }}=\sigma_{\text {cap }} \frac{\varepsilon_{\text {max }}}{\sigma_{\text {max }_{\text {tensile }}}} \cong 0.022
\end{aligned}
$$


These are the percentage increments of the length $\left(\varepsilon_{2_{\text {cylinder }}}\right)$ of the cylinder, the radius of the cylinder $\left(\varepsilon_{1_{\text {Cylinder }}}\right)$, and the radius of the spherical end cap $\left(\varepsilon_{\text {cap }}\right)$. Then, the final volume that accounts for stretching of the membrane for the pressure $P$, was $V_{1}=133.22 \mathrm{~m}^{3}$.

On the other hand, an initial estimation of the mass flow rate can be obtained from:

$$
\dot{m}=\dot{V} \rho
$$

Where, $\dot{V}$ is the volume rate and $\rho$ is the density of air. Considering $V_{1}$ and the total inflation time $\mathrm{t}=$ $200 \mathrm{sec}$, the volumetric rate is given by:

$$
\dot{V}=\frac{133.23}{200}=0.666 \frac{\mathrm{m}^{3}}{\mathrm{sec}}
$$

Substituting the numerical values into Eq. (4.8), the density of air indicated in Table 4.1, the air mass flow rate is:

$$
\dot{m}=0.666 \times 1.225=0.816 \frac{\mathrm{kg}}{\mathrm{sec}}
$$

Which is the initial air mass flow rate used for the unconfined inflation simulations described in the following sections. This air mass flow rate was identified as an initial inflator.

\subsection{Unconfined Inflation}

The flat shape obtained at the end of the controlled deflation shown in Figure 4.2(f) was used as starting point for simulation of unconfined inflation. The main purpose of the unconfined inflation was to verify and calibrate the inflator defined in the previous section. A step function was implemented to simulate the behavior of the air mass flow rate. The temperature was set equal to $288.15 \mathrm{~K}\left(15^{\circ} \mathrm{C}\right)$. In the unconfined inflation simulation, the compressive strength of the fabric material was set to $0.5 \%$ of the tensile strength and the mass scale factor was increased from 10 to 100 in order to decrease the computational time. The mass proportional damping factor $\alpha$ was set equal to 0.0 (no damping). In addition, the reference mesh was used as an initial condition to restitute the membrane to the unstressed condition existing in the nominal shape of the inflatable before the controlled deflation.

The time history of the air mass flow rate is illustrated in Figure 4.4(a). The internal (gauge) pressure $\left(P_{\text {cavity }}\right)$, the internal volume $\left(V_{\text {cavity }}\right)$ and the internal temperature $\left(T_{\text {cavity }}\right)$ are illustrated in Figure 4.4(b). 


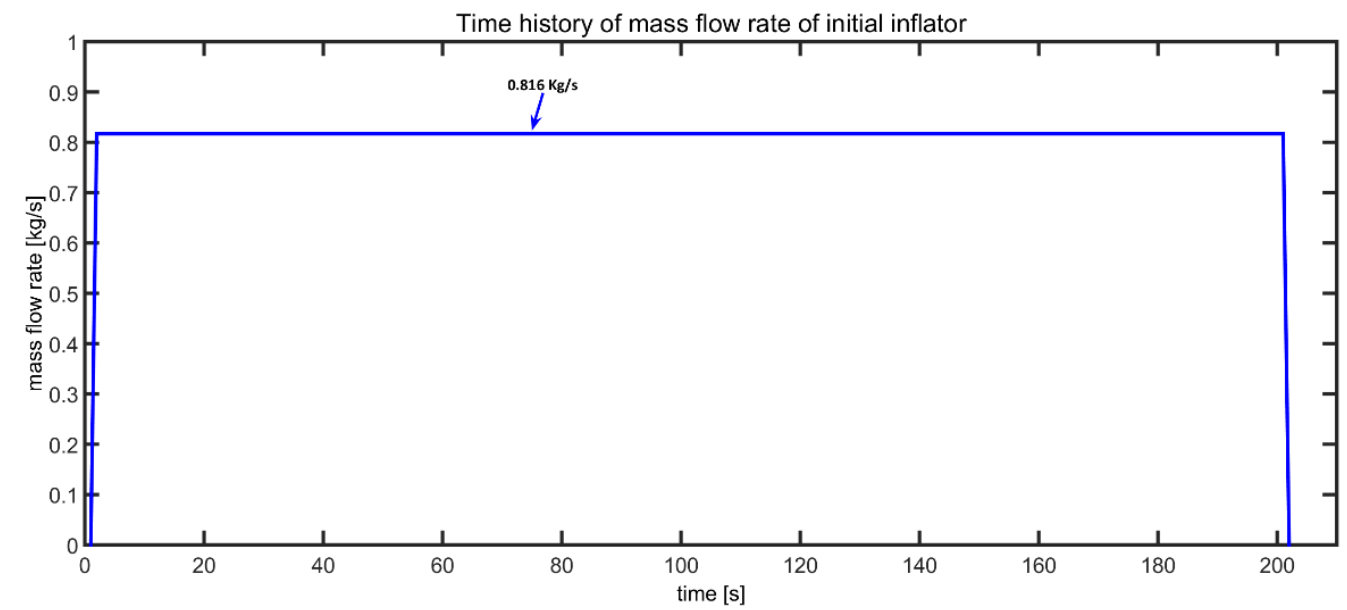

(a)

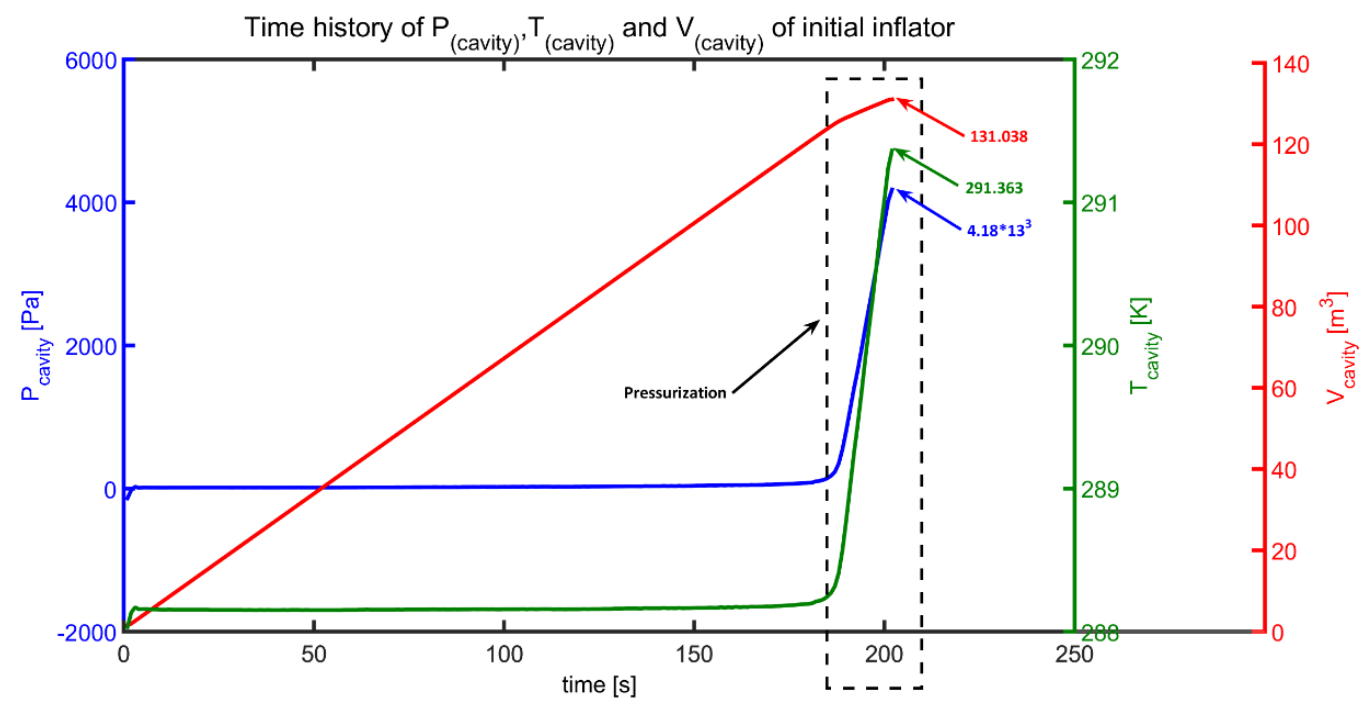

(b)

\section{Figure 4.4. (a) Air mass flow rate; (b) Time history of thermodynamic properties using the initial inflator.}

From Figure 4.4(b) it can be seen that the initially estimated air mass flow rate shown in Figure 4.4 (a) is not enough to reach the target values of pressure and volume. At the end of simulation, the values of gauge pressure and internal volume were 4.180.4 $\mathrm{Pa}$ and $131.038 \mathrm{~m}^{3}$, respectively. During the pressurization stage shown in Figure 4.4(b), the internal volume tends to a constant value while the gauge pressure increases. It is speculated that the reason for which the initial air mass flow rate was not able to reach the target values of pressure and volume, was because of the initial vacuum effect seen at the beginning of the inflation. One possible way to avoid this problem would be to extend the duration of the inflator to recover the delay produced by the vacuum effect. A second possible way would be to increase the magnitude of the 
air mass flow rate. Considering the total time specified for inflation and pressurization is fixed, it was decided to keep the duration of the inflator constant (200 seconds) and to increase the amplitude of the air mass flow rate. A few additional iterations were needed to adjust the amplitude of the air mass flow rate that was able to reach the final target values of gauge pressure and internal volume. The final adjusted magnitude of the air mass flow rate was $0.855 \frac{\mathrm{Kg}}{\mathrm{sec}}$ which is $4.8 \%$ bigger than the mass flow rate obtained using Eq. (4.10). Using this adjusted value of the air mass flow rate, the resultant gauge pressure $\left(P_{\text {cavity }}\right)$, internal temperature $\left(T_{\text {cavity }}\right)$ and internal volume $\left(V_{\text {cavity }}\right)$ are shown in Figure 4.5.

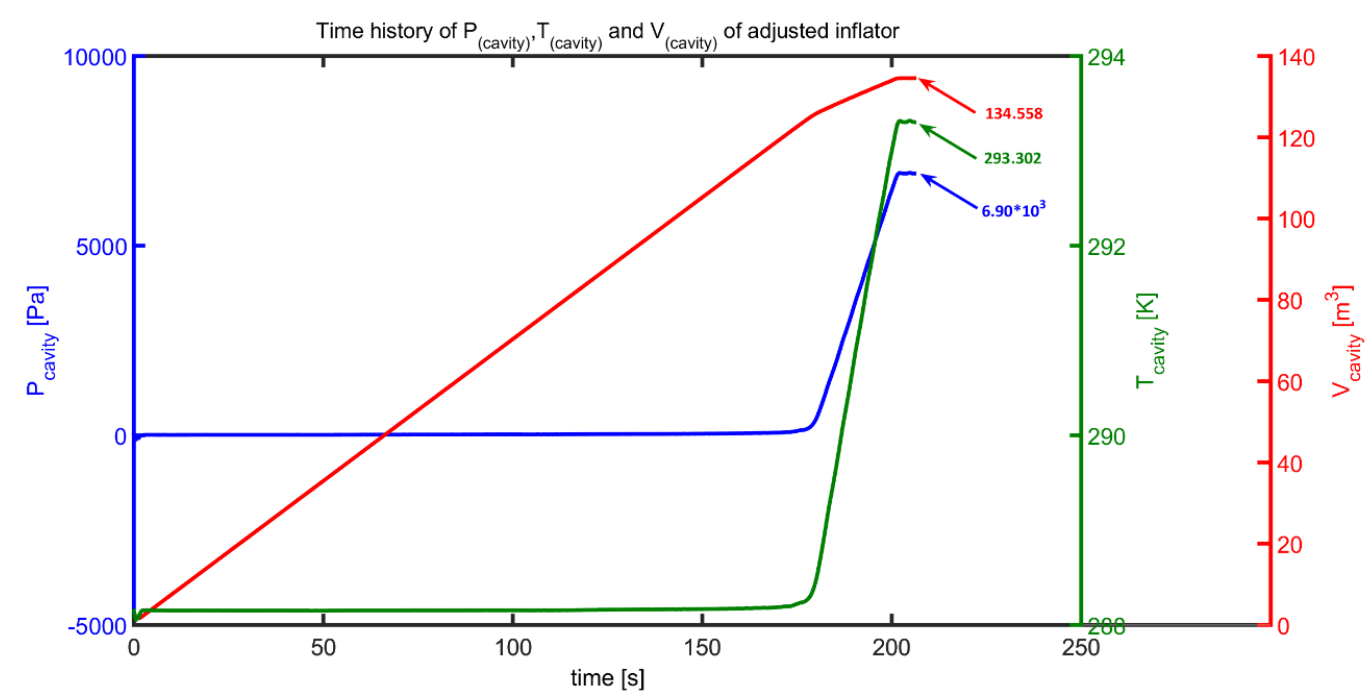

Figure 4.5. Time history of thermodynamic properties using the adjusted inflator.

\subsection{Parametric Studies for Unconfined Inflation}

The unconfined inflation that implements the adjusted inflator described in Section 4.4 was used for assessing the impact of changes in parameters in the model, and also to improve the simulation in terms of computational time and behavior of the membrane material. The parameters evaluated included the mass scale factor (MSF), the mass proportional damping factor $\alpha$ and ambient temperature $T_{a}$.

\subsubsection{Parametric Study on Mass Scale Factor}

Four different values of mass scale factor were analyzed: 1, 10,100, and 1000. From the formulation of the explicit method described in Section 2.2, the implementation of the mass scale factor produces a decrease of the computational time and at the same time an increase of inertial effects. The main purpose of this parametric study was to determine a value for the mass scale factor that was big enough to reduce the computational time of the simulations considerably but at the same time, not too big to induce high inertial 
effects not really present in the behavior of the actual structure being simulated. One of the parameters that are also influenced by the increase of the MSF is the mass proportional damping factor $\alpha$. For this reason, during this parametric study $\alpha$ was set equal to 0.0 to avoid interference from each other.

The first indicator used to evaluate the influence of the MSF was the total computational time required to complete the simulation of the unconfined inflation. The evaluation was performed using the same computer utilizing a single processor (Intel i7-4810MQ @ $2.8 \mathrm{GHz}$ ) for each value of the MSF. Each case was run separately to avoid interference during the computation of the solution. The computational times corresponding to each MSF are plotted in Figure 4.6. Results illustrated in Figure 4.6 show that the simulation with an MSF = 1 took about 339 hours (about 14 days), which is an unreasonably long time and therefore not considered for further analyses. On the other hand, an increase of MSF from 10 to 1000 reduced the computational time from about 74 hours to 14 hours. However, the reduction of computational time achieved with increasing MSF’s produced additional inertial effects described next.

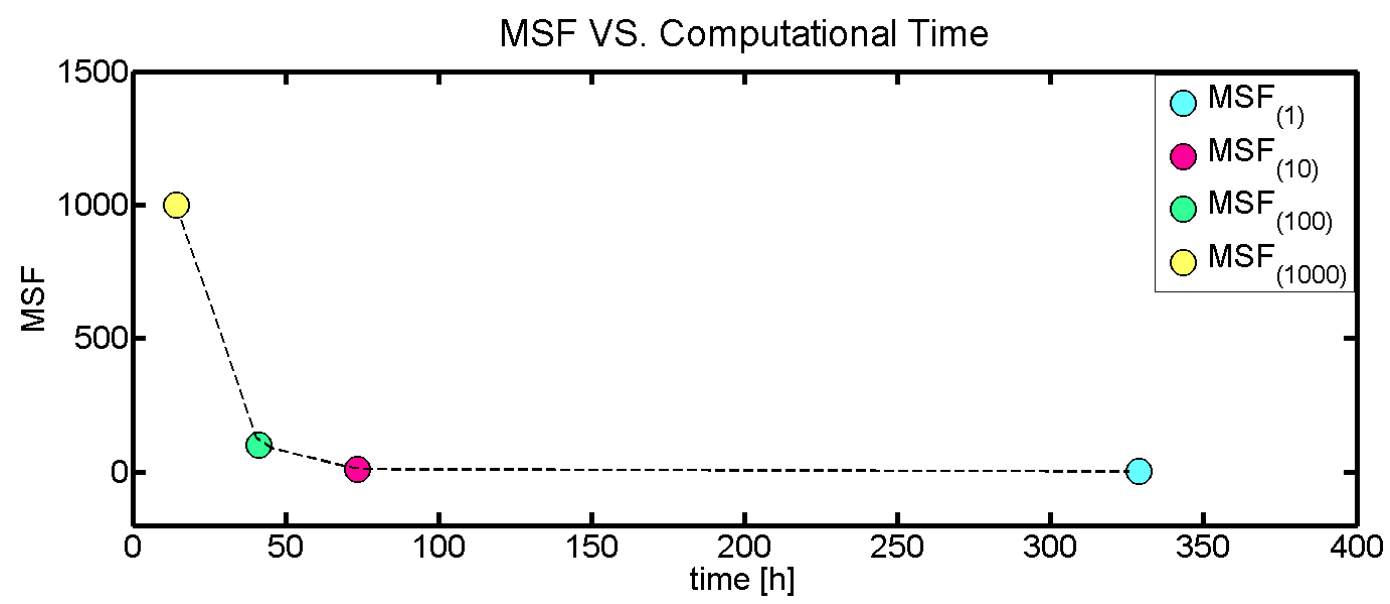

Figure 4.6. Mass Scale factor (MSF) vs. Computational Time.

The kinetic energy was used as a second indicator of the influence of the MSF. The purpose of this evaluation was to find an acceptable value to adopt for the mass scale factor for decreasing the computational time but at the same time minimizing the influence of inertial forces on the behavior of the inflatable structure.

According to the kinetic energy theorem [36], considering a system of points of mass $P_{S}\left(m_{s}\right)$ and $O x y z$ as its reference system, for a generic point of mass $P_{S}\left(m_{s}\right)$, it is possible to write the D'Alambert 's Principle:

$$
\bar{R}_{s}^{(e)}+\sum_{1}^{n} \bar{f}_{s r}-m_{s} \bar{a}_{s}=0 \quad \text { with } r \neq s
$$


where: $\bar{R}_{s}^{(e)}$ is the total external force acting on $P_{s} ; \bar{f}_{s r}$ is the internal force that $P_{r}$ acts on $P_{s}=-\bar{f}_{s r} ; \bar{a}_{s}$ is the $P_{s}$ acceleration and $-m_{s} \bar{a}_{s}$ is the inertial force of $m_{s}$. If the system is in motion and $d P_{s}$ is its infinitesimal displacement, the dot product is given by:

$$
\left(\bar{R}_{s}^{(e)}+\sum_{1}^{n} \bar{f}_{s r}-m_{s} \bar{a}_{s}\right) \times d P_{s}=0
$$

So:

$$
\bar{R}_{s}^{(e)} \times d P_{S}+\left(\sum_{1}^{n} \bar{f}_{s r} \times d P_{s}\right)-\left(m_{s} \bar{a}_{s} \times d P_{s}\right)=0
$$

The dot products of the three terms of Eq. (4.13) represent the work of external forces, the work of internal forces and the work of inertial force, respectively. If $\ddot{x}, \ddot{y}, \ddot{z}$ are the components of the acceleration $\bar{a}_{s}$, the infinitesimal work of the inertial force $-m_{s} \bar{a}_{s}$ can be written as:

$$
-m_{s} \bar{a}_{s} \times d P_{s}=-m_{s} \bar{a}_{s} \times \frac{d P_{s}}{d t} d t=-m_{s}(\ddot{x} \dot{x}+\ddot{y} \dot{y}+\ddot{z} \dot{z}) d t=-\frac{1}{2} m_{s} \frac{d}{d t}\left(\dot{x}^{2}+\dot{y}^{2}+\dot{z}^{2}\right) d t \quad \text { Eq. }
$$

since the mass is assumed to remain constant $\left(m_{s}=\right.$ constant $)$, then

$$
-m_{s} \bar{a}_{s} \times d P_{s}=-d\left(\frac{1}{2} m_{s} v_{s}^{2}\right)=-d E_{s}
$$

where $-d E_{s}$ is the change in kinetic energy.

Eq. (4.15) states that the work of inertia force is equal to the change in kinetic energy of the mass $\left(m_{s}\right)$ taken with opposite sign. Taking into account Eq. (4.13) the theorem of kinetic energy can be written using the following formulation:

$$
d L^{(e)}+d L^{(i)}=d E
$$

In the Eq. (4.16), the work $(d L)$ done by the sum of all forces (external and internal) acting on a system of points of mass in a time interval $d t$, is equal to the change in the kinetic energy $d E$ of the system in the same time interval.

Considering Equations (4.11) to (4.16), it is possible to assert that since the increase of the mass scale factor produces an increase of inertia effects, this increase produces an increase of the change in the kinetic energy as shown in Figure 4.7, particularly at the end of the inflation process. The mass scale factor artificially is increasing the magnitude of displacements, velocities, and accelerations, as described in Section 2.2, which 
in turn, produces an increase of the forces applied on the model, external and internal as well as the inertial forces.

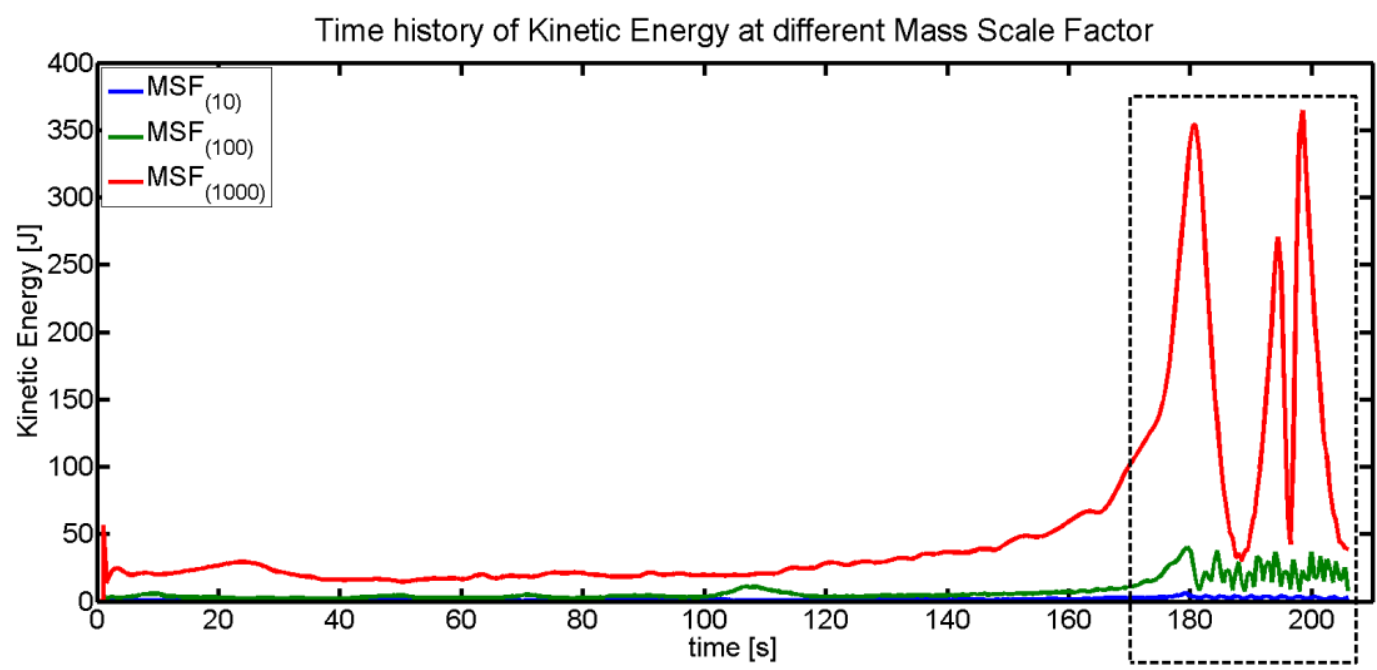

Figure 4.7. Time history of kinetic energy for different values of MSF.

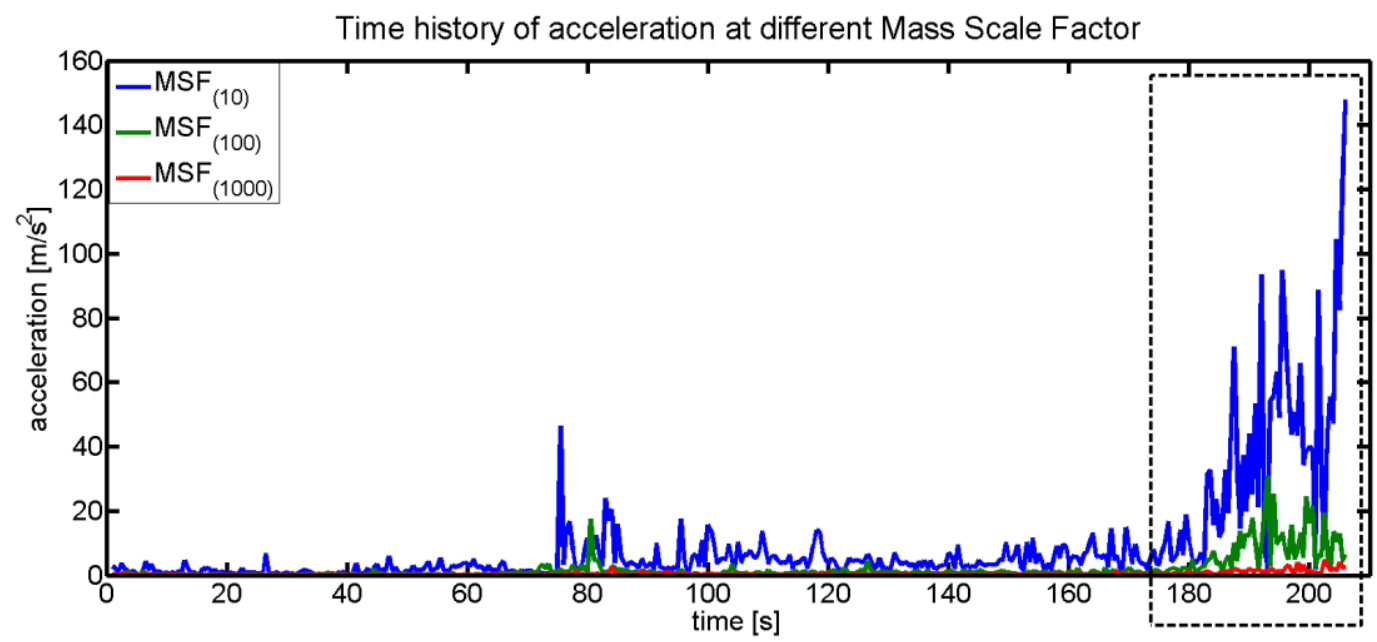

Figure 4.8. Time history of acceleration for different values of MSF.

Figure 4.7 shows that for an MSF $=10$, the amplitude of the kinetic energy is close to zero for the entire duration of the inflation process, while for an $\mathrm{MSF}=100$, the amplitude of the kinetic energy is close to zero, but it is also possible to see small oscillations at the end of the simulation (boxed area in the time interval 170 to 210 seconds). For an $\mathrm{MSF}=1000$, the kinetic energy is characterized by a remarkable increase of the amplitude with large oscillations at the end of the simulation as seen in the boxed area in the time interval 170 to 210 seconds.

Figure 4.8 illustrates the time history of the acceleration of a reference node located at the tip of the spherical end cap for different values of the mass scale factor. Looking at the magnitude of the acceleration, it can be 
seen that it decreases as the value of the mass scale factor increases. Also from Figure 4.8, it is possible to see that for almost the entire duration of the simulation the magnitude of the accelerations is close to zero. However, it is possible to notice oscillations at the end of the simulation (boxed area time interval 170 to 210 seconds) in which the amplitude of the oscillations decreased with increasing values of MSF. In the models, the effect of the increasing values of MSF is reflected in a bouncing of the inflatable structure in the last part of the inflation process, specifically during the pressurization. These results suggest that an increase of the MSF produces an effect similar to a damping effect. This effect can be explained physically; since the inertial forces act as a resistance on the motion of the inflatable, for an increasing MSF, the effect of inertial forces increase and cause a decrease in the magnitude of the acceleration of the nodes.

The stresses in the inflatable were used as a third indicator of the influence of the MSF on the models. Since the stresses distribution in the cylinder and on the spherical end caps are approximately uniform, the stresses were evaluated taking into account one node on the middle cross section of the cylindrical portion and one node located on the tip of the spherical portion of the inflatable structure. The time histories of the stresses $S_{11}$ and $S_{22}$ on the cylindrical portion during the unconfined inflation are shown in Figure 4.9 and Figure 4.10, whereas the time histories of the stresses $S_{11}=S_{22}$ on the spherical portion are shown in Figure 4.11. From the plots it can be seen that for different values of the mass scale factor the magnitude of the stresses are nearly identical as illustrated by the overlapping of the time histories. Table 4.3 summarizes the values of the stresses for different MSF captured at end of the pressurization $(t=200 \mathrm{sec})$. From the stress analysis we can conclude that the MSF doesn't have impact on the stresses in the membrane material.

Table 4.3. Maximum values of the stresses in select nodes for changing MSFs.

\begin{tabular}{|l|c|c|c|}
\hline \multirow{2}{*}{ Mass Scale Factor } & \multicolumn{2}{|c|}{ Cylinder } & End cap \\
\cline { 2 - 4 } & $\sigma_{11}[\mathrm{~Pa}]$ & $\sigma_{22}[\mathrm{~Pa}]$ & $\sigma_{11}=\sigma_{22}[\mathrm{~Pa}]$ \\
\hline $\mathrm{MSF}=10$ & $2.46 \cdot 10^{7}$ & $1.50 \cdot 10^{7}$ & $1.54 \cdot 10^{7}$ \\
\hline $\mathrm{MSF}=100$ & $2.47 \cdot 10^{7}$ & $1.49 \cdot 10^{7}$ & $1.53 \cdot 10^{7}$ \\
\hline $\mathrm{MSF}=1000$ & $2.46 \cdot 10^{7}$ & $1.51 \cdot 10^{7}$ & $1.54 \cdot 10^{7}$ \\
\hline Average & $2.46 \cdot 10^{7}$ & $1.50 \cdot 10^{7}$ & $1.54 \cdot 10^{7}$ \\
\hline
\end{tabular}




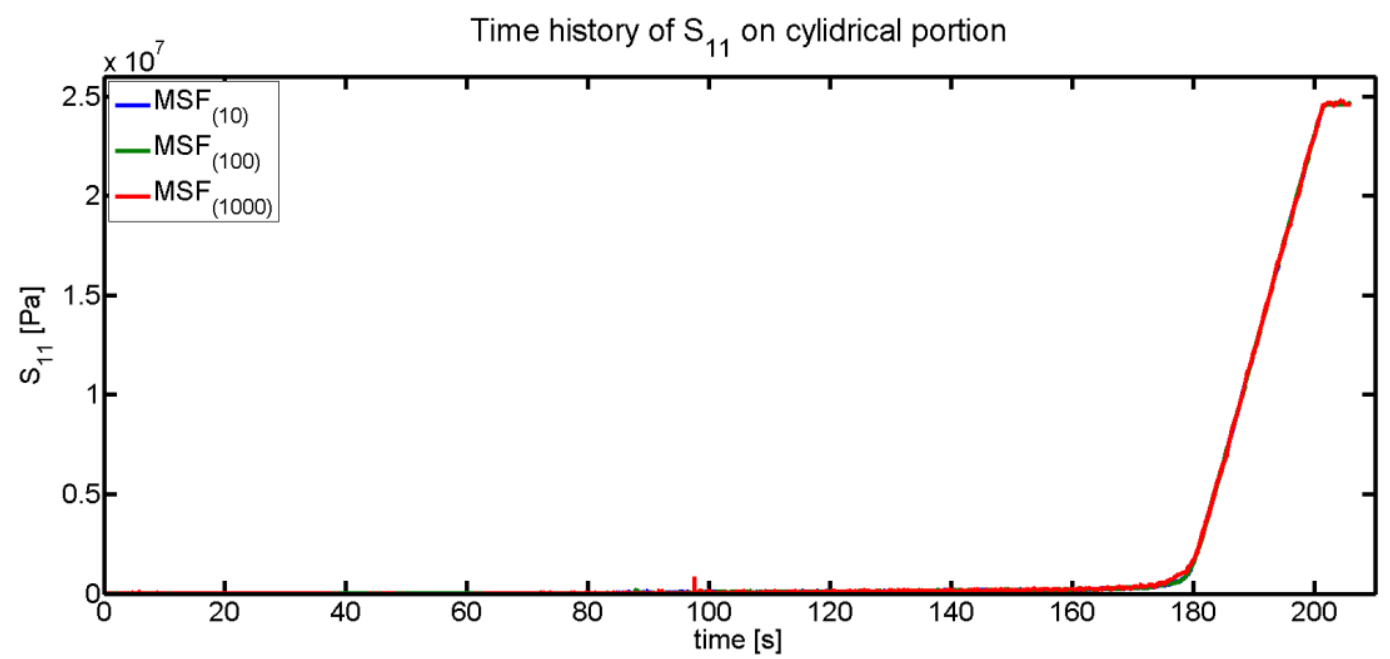

Figure 4.9. Time history of $S_{11}$ on the cylindrical portion at different values of MSF.

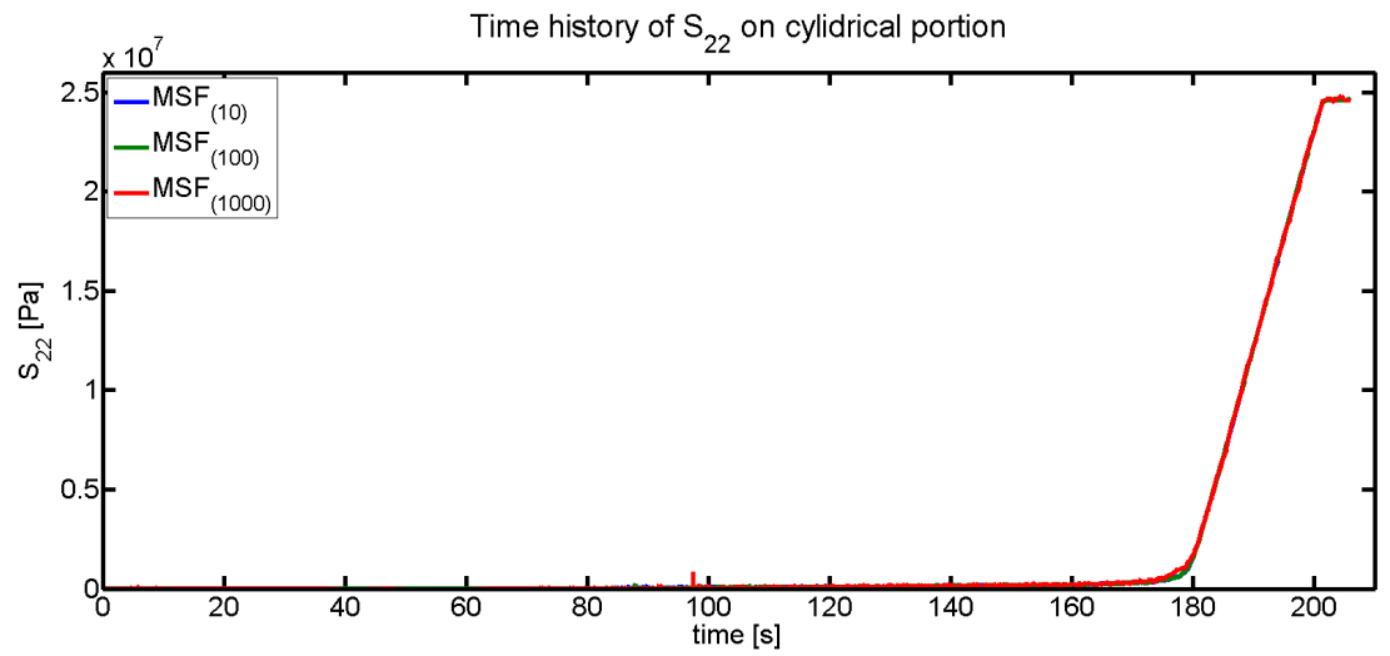

Figure 4.10. Time history of $S_{22}$ on the cylindrical portion at different values of MSF.

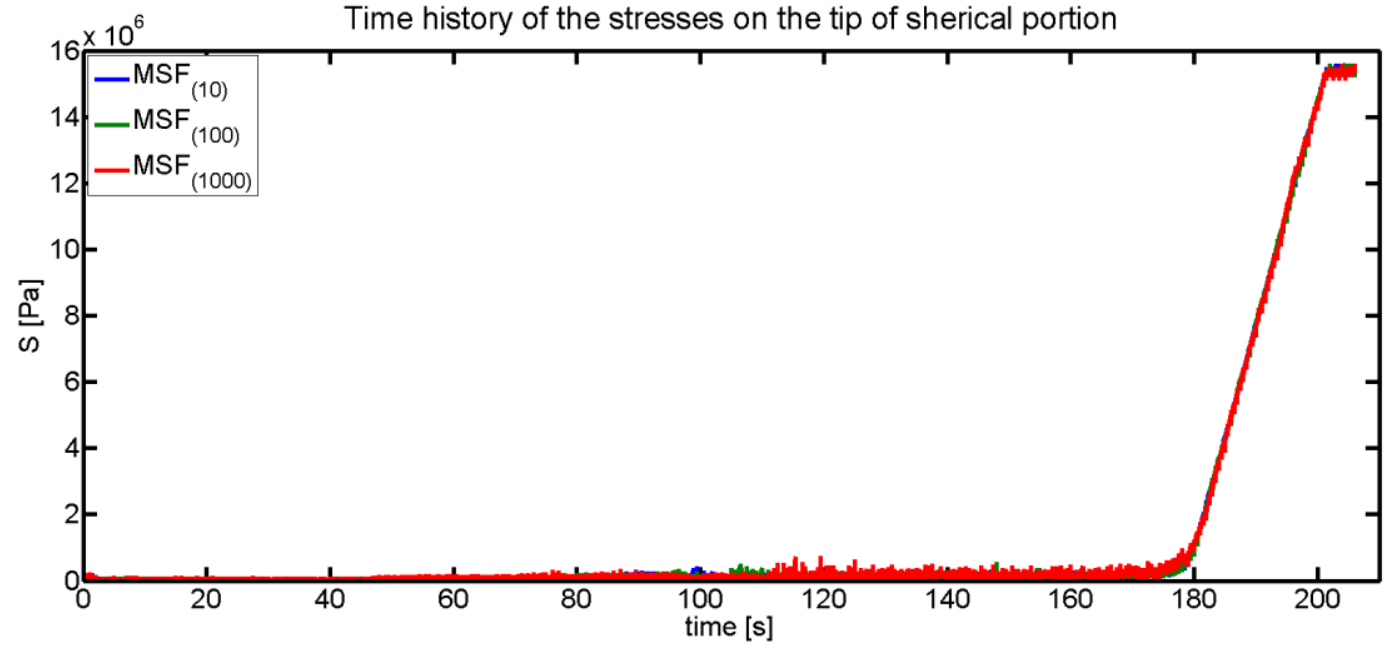

Figure 4.11. Time history of $S_{11}=S_{22}$ on the tip of spherical portion at different values of MSF. 
The results obtained from the parametric study on the influence of the MSF suggest that an MSF equal to 100 can be an acceptable value to use in the subsequent models since it is high enough to reduce the computational time to a reasonable value as shown in Figure 4.6, but at the same time it is not too high to change the behavior of the inflatable once it is fully inflated and pressurized at the target value, as shown in Figure 4.7.

\subsubsection{Parametric Study on Influence of Mass Proportional Damping Factor $\alpha$}

The inclusion of different values of the mass proportional damping factor $\alpha$ was investigated. Eight different values of $\alpha(0.0,0.2,0.4,0.6,0.8$, and 1.0) were analyzed maintaining the stiffness proportional factor $\beta$ constant and equal to $1 \cdot 10^{-6}$ for all the simulations implemented in this work. During the simulation of unconfined inflation for the different values of $\alpha$, the mass scale factor was set to 100 . In order to understand the impact of increasing values of $\alpha$, the kinetic energy was used as control output.

Results shown in Figure 4.12 indicate that the amplitude and the frequency of oscillations of the KE decrease when $\alpha$ increases. This behavior can be explained mathematically taking into account the Rayleigh damping formulation Eq. (2.11) in Section 2.4.1. Increasing the value of $\alpha$ and keeping constant $\beta$, the Rayleigh damping increases producing a decay in amplitude and in the frequency of oscillations of the KE. Not including damping in the models produces local oscillations of the membrane that can be observed on the oscillations of the KE. On the other hand, for values of $\alpha$ in the range of 0.6 to 1.0, the damping effect is significant and, there are practically no local or major global oscillations of the membrane. This behavior is similar as if the membrane is being inflated in an increasingly viscous surrounding media. This behavior doesn't seem to be realistic and therefore maintaining $\alpha$ in the range between 0.2 and 0.4 seems to reproduce a more representative behavior of the membrane.

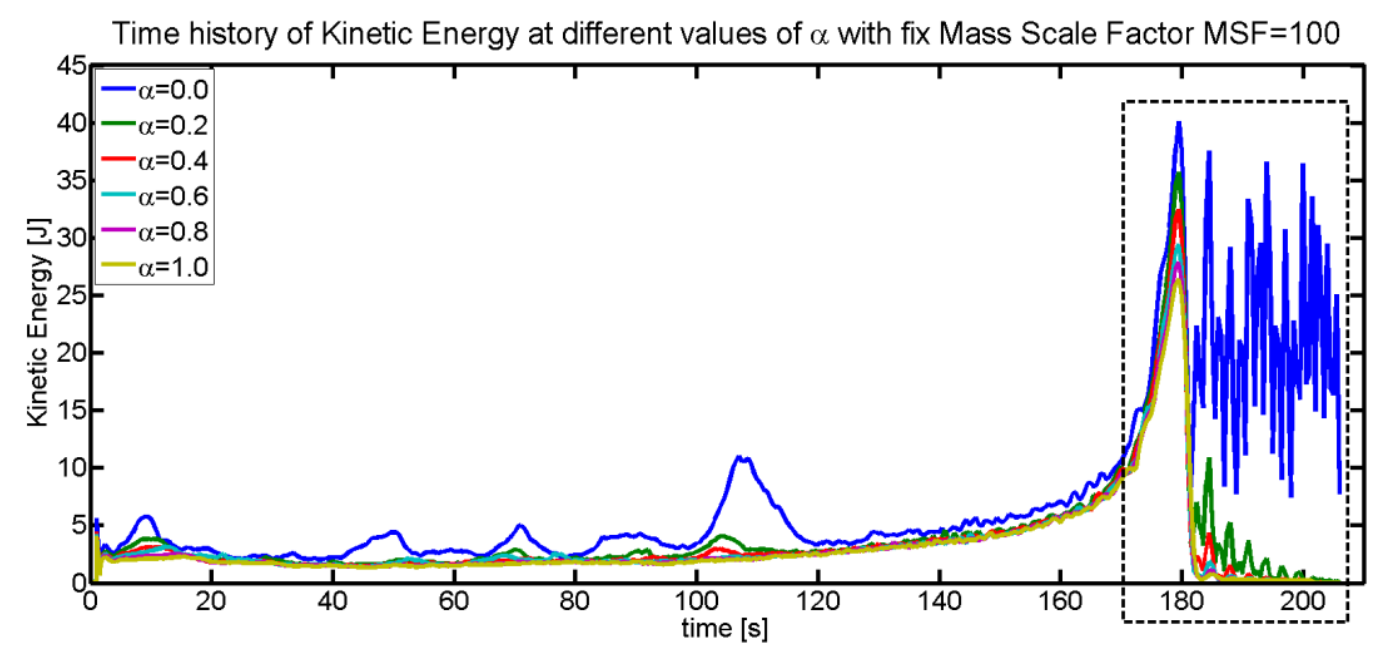

Figure 4.12. Time history of kinetic energy changing $\alpha$. 


\subsubsection{Influence of Ambient Temperature $T_{a}$ during Unconfined Inflation}

Six different values of ambient temperature $T_{a}\left(0^{\circ} \mathrm{C}, 10^{\circ} \mathrm{C}, 15^{\circ} \mathrm{C}, 20^{\circ} \mathrm{C}, 30^{\circ} \mathrm{C}, 40^{\circ} \mathrm{C}\right)$ were analyzed for an unconfined inflation. For these evaluations, the MSF was set to 100 and $\alpha$ was set to 0.0 . The Clapeyron plane [37] was used to represent the evolution of gauge pressure and volume for different temperatures. The state of the gas is represented by:

$$
P V=\gamma R T
$$

which represents one point on the Clapeyron plane. Changing one of the three parameters of the gas $(P, V, T)$ changes the position of the point. If the temperature is kept constant (isothermal transformation), we have that $P V=$ constant, then, the isothermal on the Clapeyron plane is a hyperbole. If the transformation is adiabatic, we have that $P V^{\gamma}=$ constant. In this case, the behavior of an adiabatic transformation is a hyperbole with a slope higher than the slope of an isothermal transformation. The isothermal and adiabatic behaviors are plotted in Figure 4.13 keeping the temperature constant to the target value of $15^{\circ} \mathrm{C}$. As expected, Figure 4.13 shows an increase of slope from an isothermal to an adiabatic transformation. Figure 4.14 shows the behavior of an isothermal transformation on the Clapeyron plane at different temperatures. As expected, the isothermals shift to the right of the plot as the temperature $T_{a}$ increases.

On the other hand, as shown in Figure 4.15 and Figure 4.16, increasing the ambient temperature $T_{a}$, the magnitude of the gauge pressure $\left(P_{\text {cavity }}\right)$ and the internal volume $\left(V_{\text {cavity }}\right)$ of the inflatable increased significantly. For example, an increase of ambient temperature from $T_{a}=15^{\circ} \mathrm{C}$ to $T_{a}=40^{\circ} \mathrm{C}$, produced an increase of the target pressure $P$ and target volume $V_{1}$ of $72 \%$ and $4.9 \%$, respectively. From these results it is possible to make an important observation: considering that the adjusted inflator was evaluated using an ambient temperature of $T_{a}=15^{\circ} \mathrm{C}$, results indicate that an increase of ambient temperature accelerates the increase of pressure and also exceeds the target values of $P$ and $V_{1}$, as shown in Figure 4.15. In order to avoid this effect, and according to Eq.(4.17), the inflation system would need to be adjusted to decrease the air mass flow rate as the ambient temperature increases to avoid excessive pressurization of the inflatable that would produce excessive stretching of the membrane and ultimately lead to material failure. 


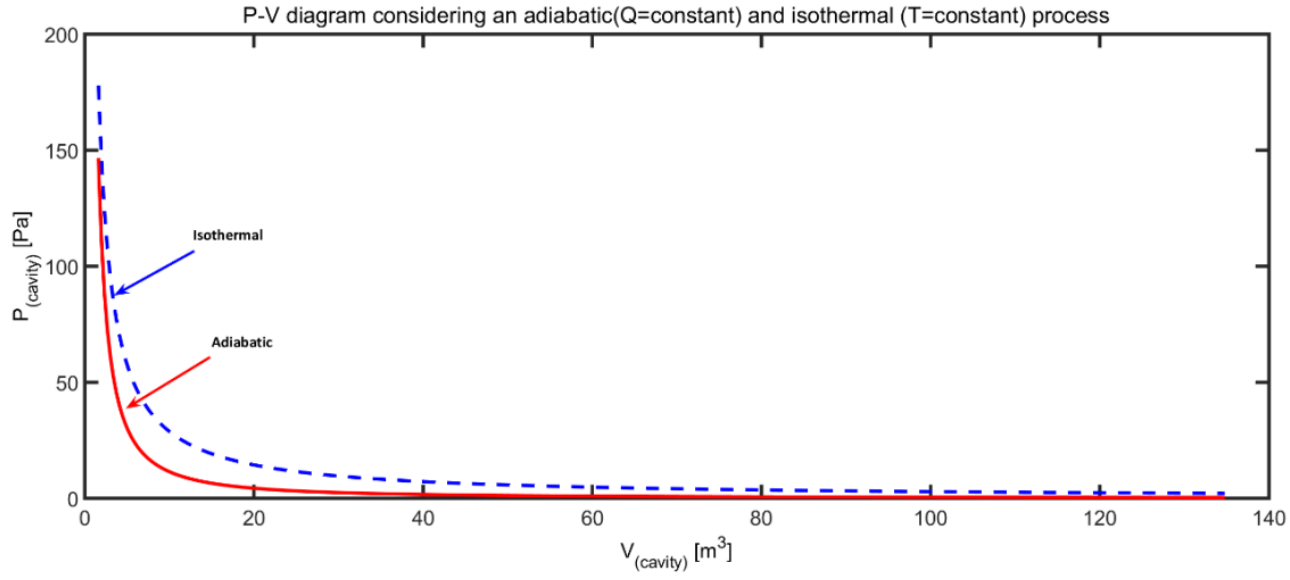

Figure 4.13. Adiabatic and isothermal transformation on the Clapeyron plane at $T_{a}=15^{\circ} \mathrm{C}$.

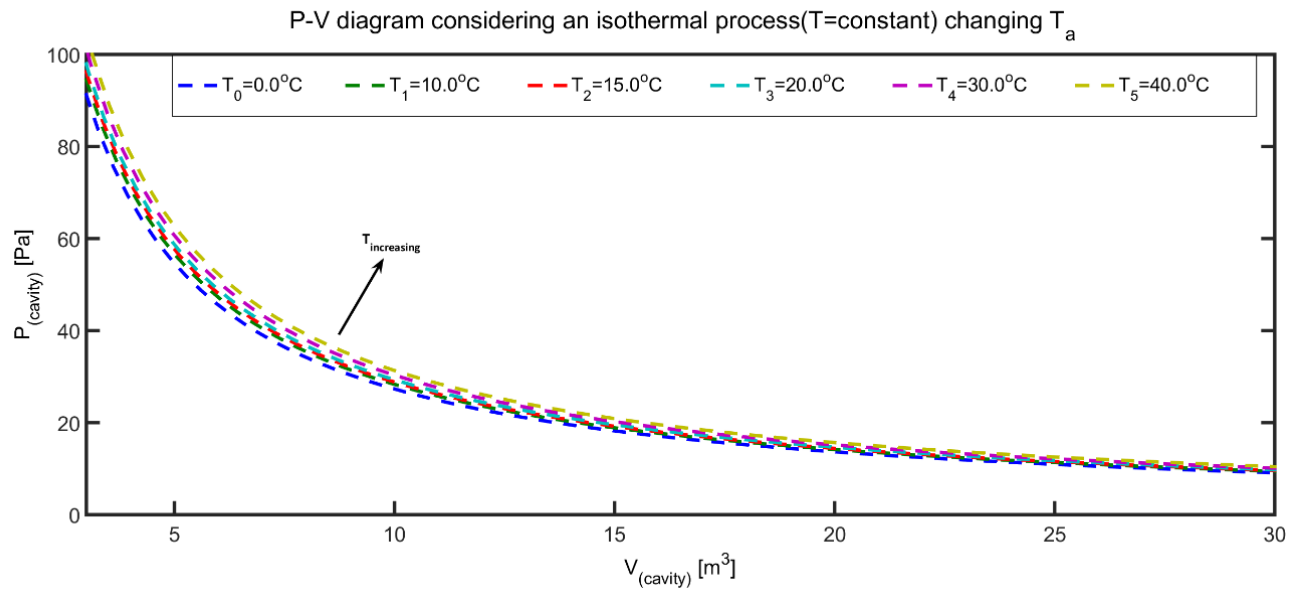

Figure 4.14. Isothermal transformations on the Clapeyron plane for increasing $T_{a}$.

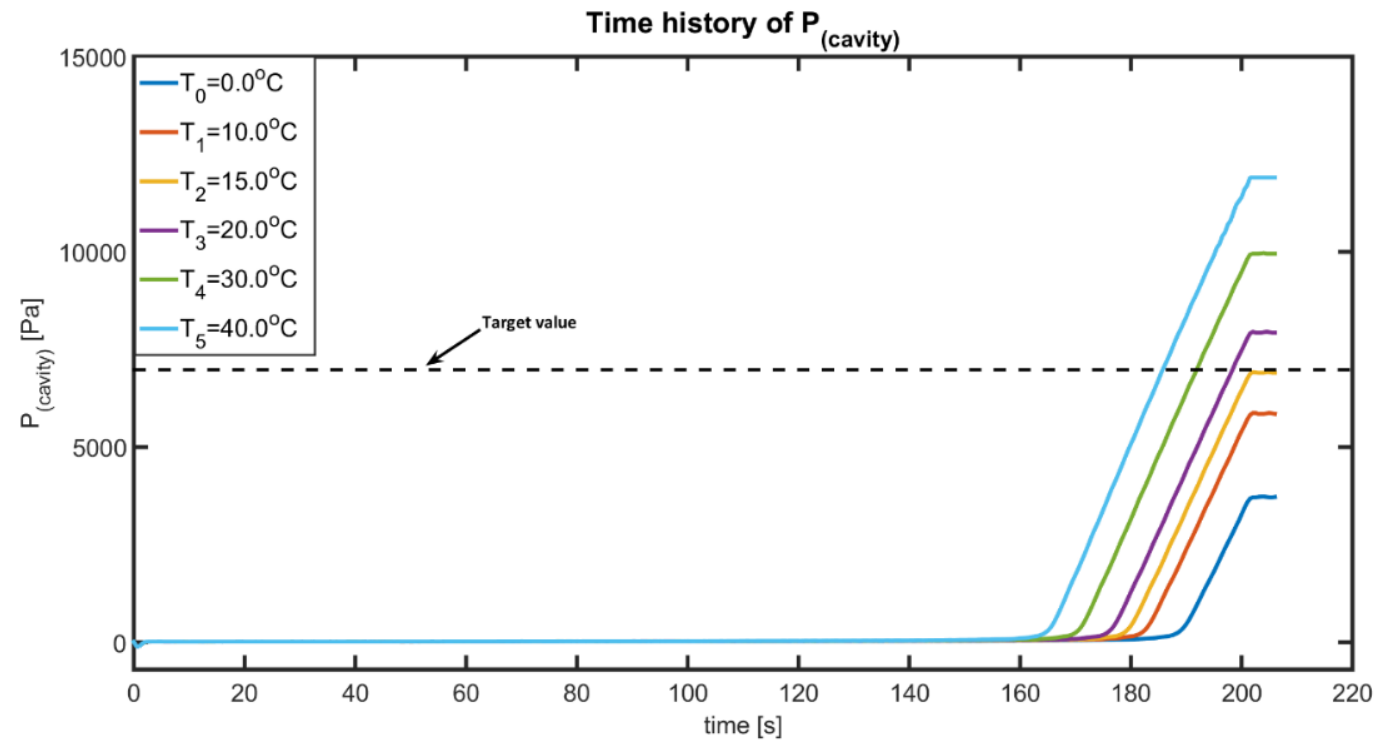

Figure 4.15. Time history of gauge pressure $\left(P_{\text {cavity }}\right)$ changing $T_{a}$. 


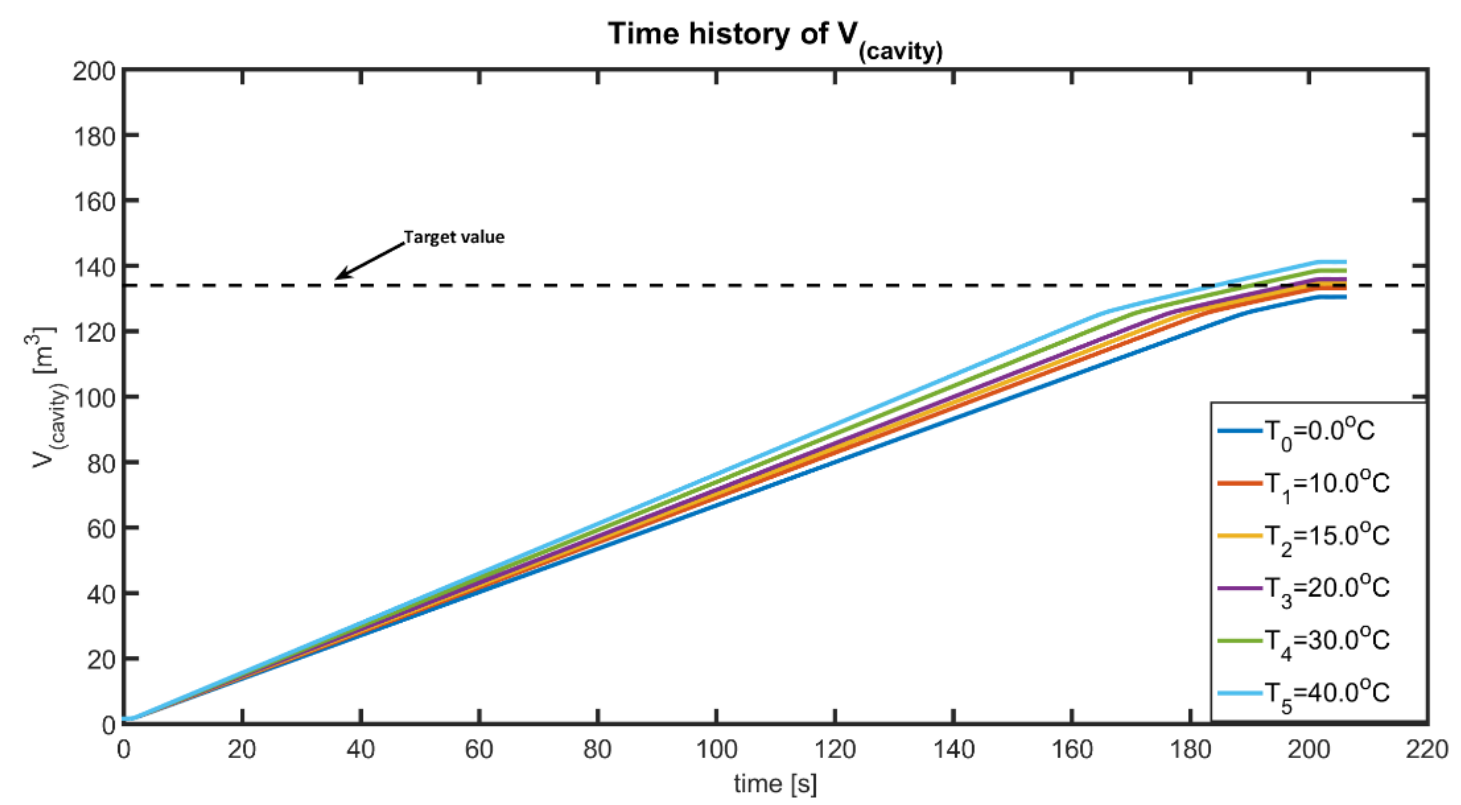

Figure 4.16. Time history of $\left(V_{\text {cavity }}\right)$ changing $T_{a}$.

\subsection{Summary}

Two techniques for controlling the deflation of the nominal shape of the inflatable structure were presented in this chapter; the uncontrolled deflation and the controlled deflation. Although the controlled deflation required more iterations compared to the uncontrolled deflation, its implementation produced a remarkable improvement in the resultant flat shape in terms of the size and distribution of wrinkles.

The simulation of unconfined inflation was introduced in this chapter as well. The simulation of the inflation required the definition of an inflator system. The gas used for this inflator and its thermodynamic properties were presented. An initial estimation of the air mass flow rate needed to fill the internal volume of the inflatable structure was conducted, and it was identified as an initial inflator. The nominal internal volume was adjusted to account the stretch of the membrane under the effect of internal pressure.

The flat shape obtained at the end of the controlled deflation was used to perform the unconfined inflation and to test the initial inflator. Since the initial inflator was not enough to achieve the target values of pressure and volume, additional iterations were needed to find the value of mass flow rate able to reach the target values, and it was identified as the adjusted inflator. Using the adjusted inflator, the unconfined inflation was used as a tool to assess the impact of changes in parameters such as mass scale factor (MSF), the mass proportional damping factor $(\alpha)$ and ambient temperature $\left(T_{a}\right)$.

Four different values of $\operatorname{MSF}(1,10,100$, and 1000) were analyzed. Since using an MSF=1 the simulation took approximately 14 days to complete, that case was not considered for the analyses. The results obtained in the parametric study of the MSF showed that increasing MSF's did not have an impact on the stresses 
on the membrane material. The parametric study indicated that an MSF equal to 100 was an acceptable value to use in the models since it was high enough to reduce the computational time but at the same time not too high to change the behavior of the inflatable once it was completely inflated and pressurized.

The influence of different values of mass proportional damping factor $\alpha(0.0,0.2,0.4,0.6,0.8$, and 1.0) were analyzed. For these evaluations, the mass scale factor was set to 100 . The kinetic energy was used as control output in order to understand the impact of increasing values of $\alpha$ could have on the behavior of the unconfined inflation. Results showed that the amplitude and the frequency of oscillations at KE decreased when $\alpha$ increased. If the mass proportional damping $\alpha$ was not included in the model, the KE was characterized by an increase of the amplitude with large oscillations at the end of the pressurization. Values of $\alpha$ in the range of 0.6 to 1.0, practically eliminated local and major global oscillations whereas maintaining $\alpha$ in the range between 0.2 and 0.4 produced a more realistic behavior of the membrane.

A parametric study was also conducted to understand the impact of a change in the ambient temperature $T_{a}$ could have on the simulation of unconfined inflation. Six different values of ambient temperature $T_{a}$ $\left(0^{\circ} \mathrm{C}, 10^{\circ} \mathrm{C}, 15^{\circ} \mathrm{C}, 20^{\circ} \mathrm{C}, 30^{\circ} \mathrm{C}, 40^{\circ} \mathrm{C}\right)$ were analyzed. During the simulation the mass scale factor was set equal to 100 and $\alpha$ equal to 0.0 . Results indicated that the gauge pressure and internal volume of inflatable structure, increased significantly as the value of $T_{a}$ increased. 


\section{Chapter 5. Confined Inflation - Uncontrolled Membrane Release}

\subsection{Introduction}

Starting from the flattened shape of the inflatable structure obtained at the end of the controlled deflation technique, this chapter describes the procedure adopted for folding the flattened membrane. The maneuvers needed for positioning of the folded shape in the storage area on the ceiling of the tunnel are described as well. The chapter also presents an initial estimation of the air mass flow rate for the inflator implemented in the simulation of confined inflation. The simulation of confined inflation is performed to understand the behavior of the membrane during the inflation inside of the tunnel segment and to verify that target values of pressure and volume are reached. This chapter ends with a parametric study on the influence of stiffness of the membrane material on the global behavior of the inflatable during the initial deployment and inflation.

\subsection{Folding Process}

The folding sequence presented in this section included the definition of two rotating planes (FP1, FP2) and two translational planes (FP3, FP4) as shown in the top left view of Figure 5.1.

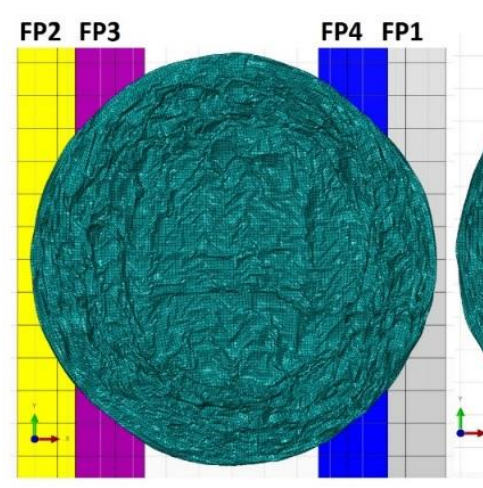

(a)

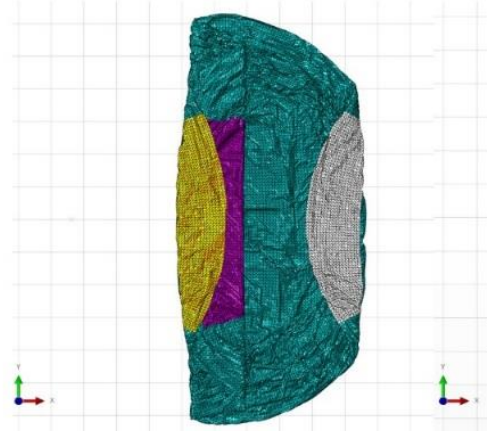

(d)

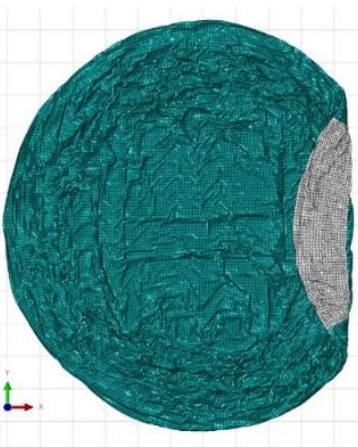

(b)

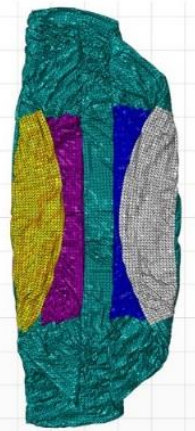

(e)

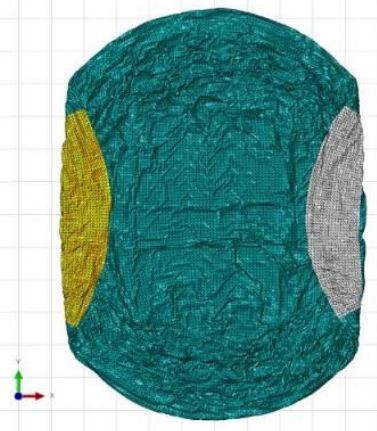

(c)

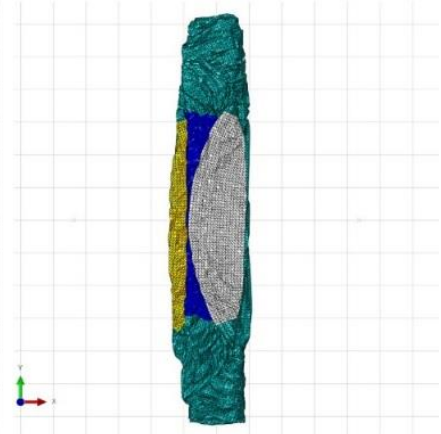

(f)

Figure 5.1. Folding sequence, main folding steps, top view (folding planes removed for clarity). 
The dimensions of the planes were adjusted according to the dimensions of the completely deflated shape obtained at the end of the controlled deflation as shown in Figure 4.3 (after application of gravity). The folding sequence illustrated in Figure 5.1 was completed in thirteen simulation steps in order to achieve the final folded shape. The position of the folding planes was selected in order to perform a symmetric folding sequence. In Figure 5.1, the colors of the folds correspond to the colors of the folding planes that were used to perform a specific folding step. All the steps of the folding sequence were performed using an MSF $=$ 100 , a mass proportional damping factor $\alpha=0.0$ and a contact penalty factor $\mathrm{CPF}=30$.

The sequence of steps performed to reach the folded shape illustrated in Figure 5.2 included:

1) In the first simulation step shown in Figure 5.2(a), the inflatable structure was initially flattened by the action of gravity applied to all membrane elements along the Z-axis. After this initial step gravity was active for the total duration of the folding process. Contact interactions and friction were defined between the membrane of the inflatable and the base $(\mu=0.50)$, between the membrane of the inflatable and the folding planes $(\mu=0.50)$ and between the membrane of the inflatable and itself $(\mu=$ 0.50). The contact interactions between folding planes and the inflatable were activated and deactivated during the different folding steps. No contact interactions were assigned between the rigid bodies (base and folding planes). The folding sequence started by imposing translational and rotational boundary conditions to the reference nodes of the folding planes that acted as rigid bodies.

2) In the second simulation step shown in Figure 5.2 (b), the reference node of the folding plane FP1 was set to translate vertically along the Z-axis $\left(U_{3}=0.20 \mathrm{~m}\right)$. This vertical translation along the Z-axis is part of the first step of the first fold.

3) In the third simulation step shown in Figure 5.2 (c), the reference node of the folding plane FP1 was set to translate horizontally along the $\mathrm{X}$ axis $\left(U_{1}=0.20 \mathrm{~m}\right)$. This horizontal translation along the $\mathrm{X}$ axis is part of the second step of the first fold.

4) In the fourth simulation step shown in Figure 5.2 (d), the reference node of the folding plane FP1 was set to rotate around the Y-axis $\left(U_{5}=90^{\circ}\right)$. At the end of this step the first fold was completed.

5) From the fifth to the seventh simulation step, the second fold was performed by the folding plane FP2 which followed the same steps of FP1, but its horizontal translation and rotation were performed in opposite directions in order to achieve the second fold symmetric to the previous one, and it is shown in Figures 5.2 (e) and Figure 5.2 (f).

6) In the eighth simulation step shown in Figure $5.2(\mathrm{~g})$, the reference node of the folding plane FP3 was set to translate vertically along the $\mathrm{Z}$-axis $\left(U_{3}=0.25 \mathrm{~m}\right)$. This vertical translation was the first step of the third fold. 
7) In the ninth simulation step shown in Figure $5.2(\mathrm{~h})$, the reference node of the folding plane FP3 was set to translate horizontally along the $\mathrm{X}$ axis $\left(U_{1}=1.8 \mathrm{~m}\right.$ ). This horizontal translation was the second step of the third fold.

8) In the tenth simulation step shown in Figure 5.2 (i), the contact interaction between the folding plane FP3 and the membrane of the inflatable structure was removed in order to complete the third fold.

9) The fourth fold was performed by the folding plane FP4 which followed the same steps of the folding plane FP3, but in the opposite direction in order to achieve the fourth fold symmetric to the previous one and it is shown in Figure $5.2(\mathrm{j})$ and Figure $5.2(\mathrm{k})$.

10) At the end of the folding process, the final folded shape shown in Figure $5.2(\mathrm{k})$ was exported to Abaqus/CAE and to Hypermesh to inspect the mesh and detect if the membrane elements were affected by inter-element penetrations and intersections, and in such case, correct them before proceeding to perform the placement of the folded shape in the storage area on the ceiling of the tunnel.

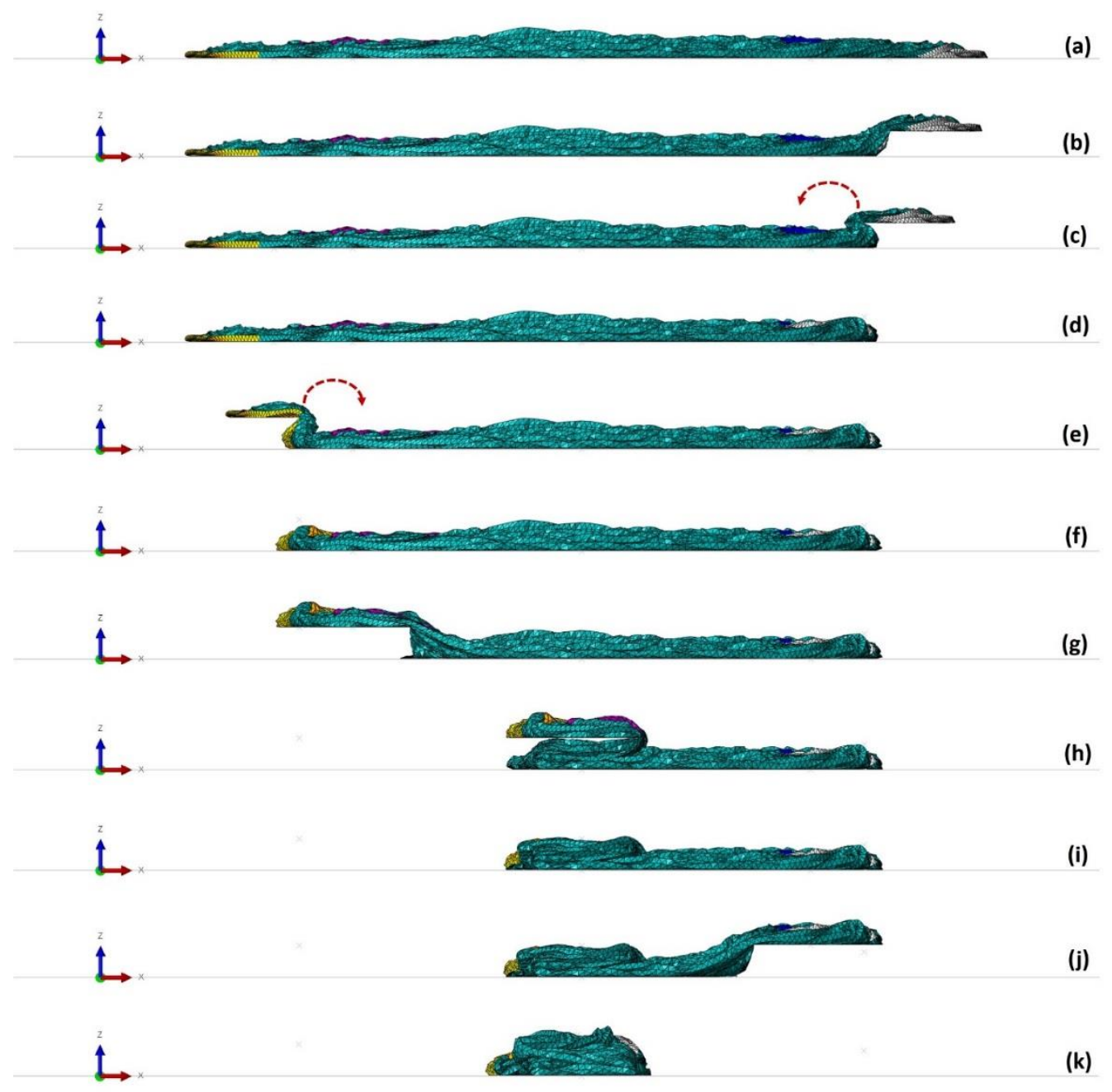

Figure 5.2. Folding sequence, main folding steps (lateral view). 


\subsection{Placement Process}

The folded shape obtained at the end of the folding sequence described in the previous section was used to perform the placement inside the tunnel segment illustrated in Figure 5.3. At the beginning of the placement process, the folded shape was pre-positioned at the center of the tunnel, as illustrated in Figure 5.3(a) and it was defined as a rigid body. The placement process was performed in two simulation steps by imposing rotational and translational boundary conditions to the reference node of the folded shape.

During the first step of the simulation, the reference node of the folded shape was set to rotate around the Y-axis $\left(U_{5}=180^{\circ}\right.$ ) in order to turn the folded shape around so that the flatter shape can be used for attachment to the ceiling of the tunnel, as shown in Figure 5.3(c). During the second step of the simulation, the reference node was set to translate vertically along the $\mathrm{Z}$ axis $\left(U_{3}=2.41 \mathrm{~m}\right)$ in order to complete the positioning onto the ceiling of the tunnel as shown in Figure 5.3(e). Figures 5.3(b) and 5.3(d) show additional intermediate steps for better illustration of the placement procedure.

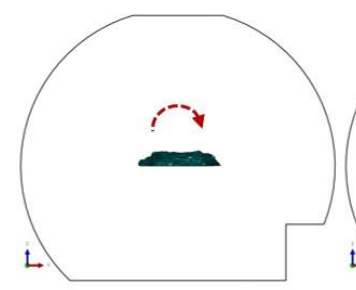

(a)

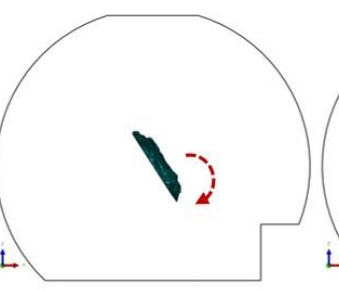

(b)

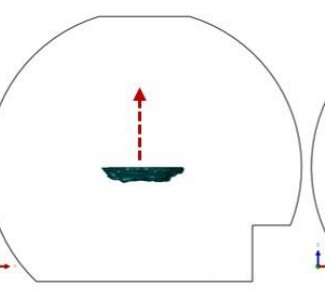

(c)

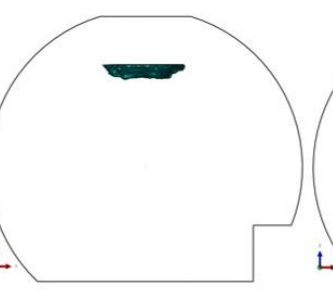

(d)

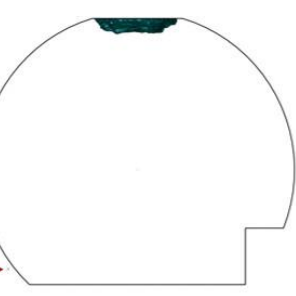

(e)

Figure 5.3. Placement process.

\subsection{Inflator Design for Confined Inflation}

The procedure explained in Section 4.3 was used for the design of the inflator for confined inflation. The initial estimation of the internal volume adopted for the confined inflation included two main parts: a) a cylindrical portion evaluated taking into account the volume of a segment of the tunnel with a circular crosssection with a radius of $2.51 \mathrm{~m}$ and a length equal to the same length of the nominal cylindrical portion of the inflatable and equal to $3.66 \mathrm{~m}$; b) the nominal volume of the spherical end caps plus an increment of the volume due to the stretch of the membrane due to pressurization.

The volume of the cylindrical portion of the inflatable in the tunnel is given by:

$$
V_{T}=\pi \cdot(2.51)^{2} \cdot(3.66)=72.44 m^{3}
$$


The volume of the spherical end caps is:

$$
V_{\text {Total }(\text { end-caps })}=V_{(\text {end-caps })}+\Delta V_{(\text {end-caps })}=35.74 \mathrm{~m}^{3}
$$

Where $\Delta V_{(\text {end-caps })}$ is the increment of the volume due to the stretching of the membrane during pressurization discussed in Section 4.3.1. Then, the final estimated internal volume of the inflatable under confined conditions is given by:

$$
V_{(\text {internal volume })}=V_{T}+V_{\text {Total }(\text { end-caps })}=108.2 \mathrm{~m}^{3}
$$

Substituting the value of the internal volume obtained using Eq. (5.3) into Eq. (4.8), the air mass flow rate

for confined inflation was estimated to be $0.66 \frac{\mathrm{kg}}{\mathrm{sec}}$. This air mass flow rate is identified as initial inflator for confined inflation.

\subsection{Initial Deployment and Confined Inflation}

The sequence of deployment and inflation started with the folded shape positioned in the storage area on the ceiling of the tunnel as described in Section 5.3 and shown in Figure 5.3(e). The tunnel was assumed to be a rigid body fixed in the $\mathrm{X}, \mathrm{Y}$, and $\mathrm{Z}$ global directions. The simulation of the initial deployment followed by the inflation was performed in one step in which gravity and the inflator system were activated sequentially. Gravity was applied as an impulse at the beginning of the simulation, and the inflator was activated with 2 seconds of delay in order to reproduce experimental results reported in [7]. During the deployment, it was necessary to call the reference mesh so that it was able to restitute the membrane to the unstressed condition existing before the controlled deflation and the folding process. The folded shape was connected to the ceiling of the tunnel using three lines of nodes defined along the cylindrical portion as shown in Figure 5.4. During the deployment, the nodes of attachment lines were not allowed to translate but were allowed to rotate. These nodes represented the ties that fastened and restrained the inflatable structure to the ceiling of the tunnel.

The confined inflation was performed using an MSF of 100, a mass proportional damping factor $\alpha=0.2$ and a CPF of 10. Contact interactions and contact properties as friction were defined between the membrane of the inflatable and the tunnel $(\mu=0.40)$ and between the membrane of the inflatable and itself $(\mu=$ 0.20). The entire initial deployment and inflation sequence was set to take place in 200 seconds plus 5 additional seconds for inflation deactivation and pressure stabilization. The initial inflator, with an air mass flow rate of $0.66 \frac{\mathrm{kg}}{\mathrm{sec}}$, was implemented for an initial evaluation of confined inflation. 


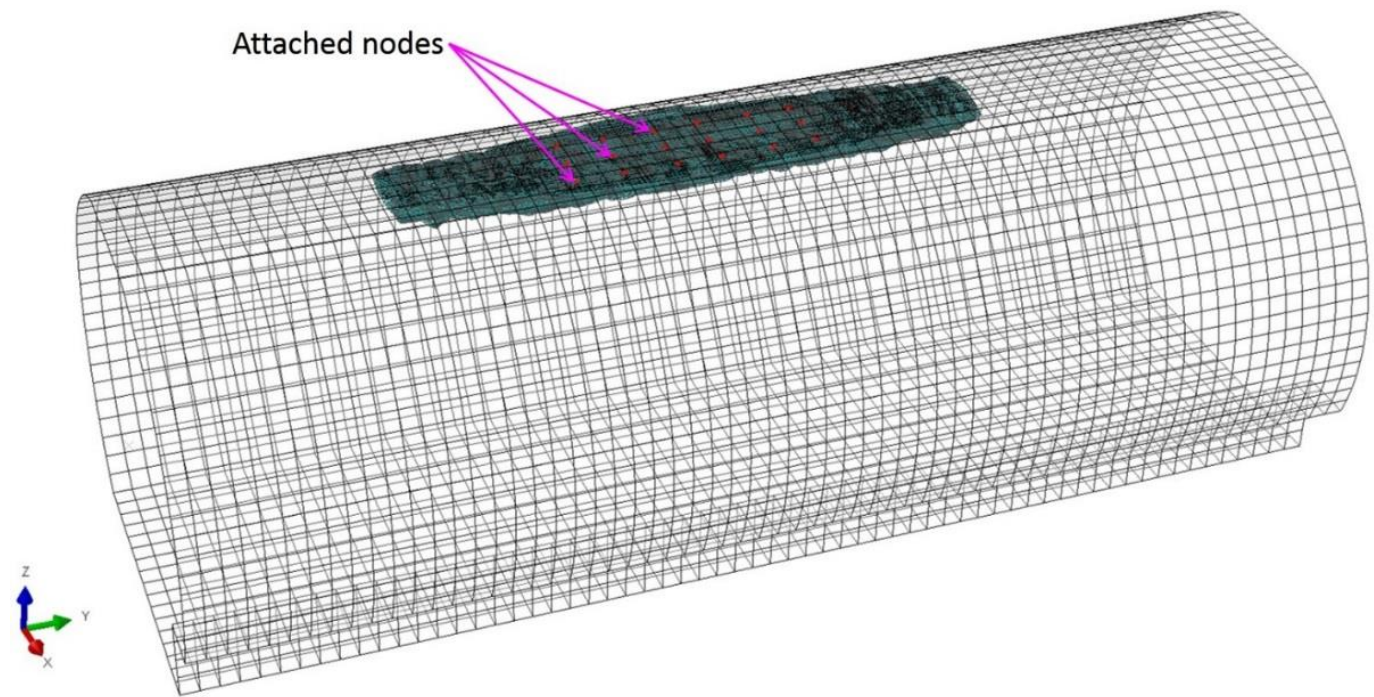

Figure 5.4. Detail of attached lines in the ceiling of the tunnel.

A sequence of images showing the initial deployment from the ceiling of the tunnel and subsequent inflation is illustrated in Figure 5.5. These images include simulation results compared to experimental results reported in [7]. The simulation started with the folded inflatable on the ceiling of the tunnel as shown Figure 5.5(a). The initial unfolding and fall of the inflatable structure from the ceiling of the tunnel were induced by the self-weight of the membrane during the first two seconds, as illustrated in Figure 5.5(b). Then, the activation of the inflator contributed to continuing the unfolding process until the membrane of the inflatable structure reached the floor of the tunnel. The inflator continued adding air mass for 200 seconds producing expansion of the membrane (Figures 5.5(c) to 5.5(e)) until the inflatable structure was fully inflated within the tunnel segment, as illustrated in Figure 5.5(f).

Full-Scale Experiment

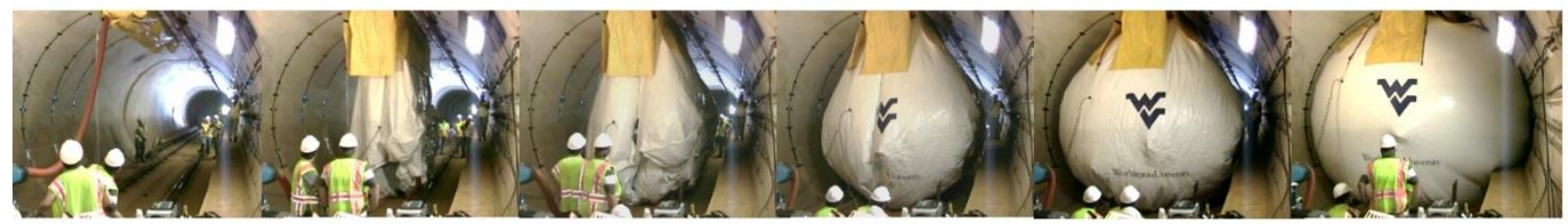

FE Model: Confined Inflation

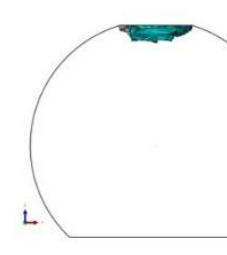

(a)

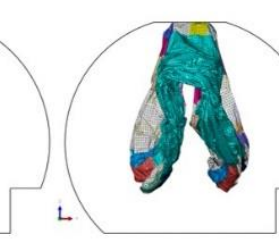

(b)

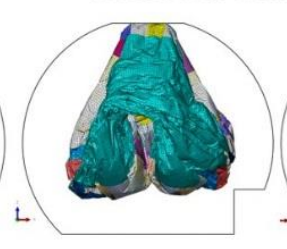

(c)

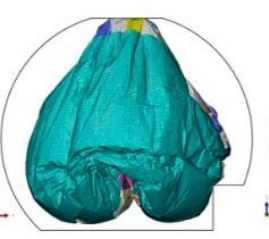

(d)

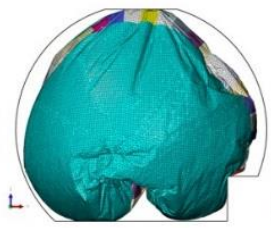

(e)

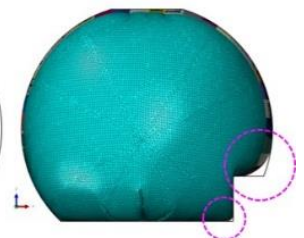

(f)

Figure 5.5. Results of FE Model compared to full-scale experiments reported in [7]. 
Although the sequence of images of Figure 5.5 followed the sequence seen in the experimental test, the behavior of the membrane material in the simulation did not totally reflect the behavior observed in the experiments. From the sequence shown in Figure 5.5, the membrane seems to have less flexibility than the flexibility observed in the actual experimental prototype. This behavior is attributed to the artificial compressive strength adopted in the definition of the membrane material. In the model of Figure 5.5, the compressive strength was $0.5 \%$ of the maximum tensile strength for a strain $\varepsilon=0.014$. A parametric evaluation of the influence of the compressive strength was carried out and the results are described in Section 5.5.6.

Considering the global conformity of the inflatable to the tunnel, the inflated shape at the end of the simulation was similar to shape observed in the experiments. However, considering the local conformity, the simulation showed that the inflatable was not able to conform to at least two corners of the tunnel profile, as shown in Figure 5.5(f). A detailed view of the lack of local conformity is illustrated in Figure 5.6(b), which shows two clear contact gaps on the right corners of the tunnel profile. The formation of the gaps is attributed to the lack of uniform distribution of the membrane material which is accumulated on tunnel floor and was not able to be transferred to the lateral portions of the tunnel by the action of the inflation pressure, as shown in Figure 5.6(a).

A possible way to improve the membrane distribution in the simulations is to produce a gradual release of membrane material during the inflation process as carried out in the experiments [7-14]. In order to produce a gradual release of the membrane, a better control of the membrane was implemented during the deflation by introducing pre-folds held by passive restrainers, as explained in Chapter 6 .

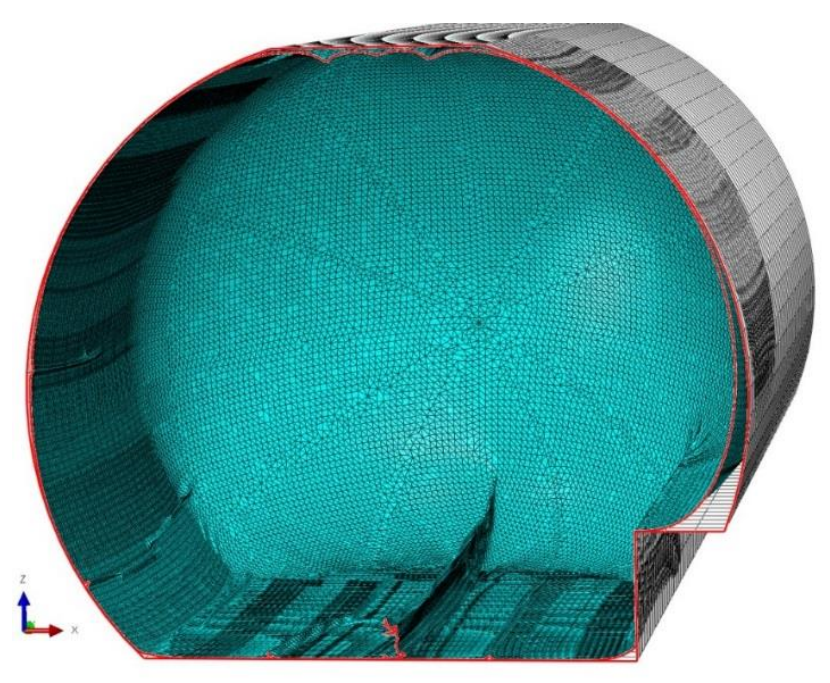

(a)

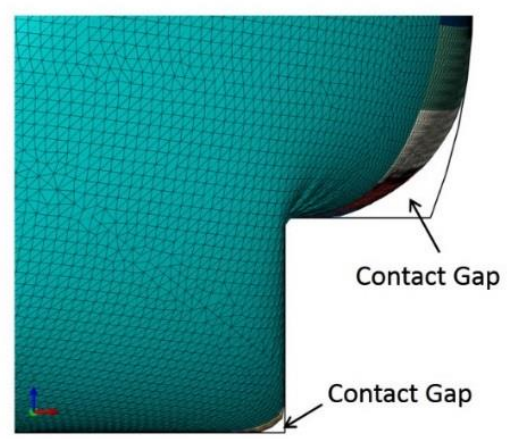

(b)

Figure 5.6. FE Model: (a) Detail of wrinkles on the tunnel floor; (b) Detail view of local conformity. 
The performance of the initial inflator during the confined inflation is shown in Figure 5.7. Results indicate that the initial inflator produced at the end of the inflation values of the gauge pressure and internal volume close to the target values. The gauge pressure reached a value of $6.60 \cdot 10^{3} \mathrm{~Pa}$ which is $4.3 \%$ lower than the target value of $6.89 \cdot 10^{3} \mathrm{~Pa}$. The internal volume reached a value of $103 \mathrm{~m}^{3}$, which is $4.6 \%$ lower than the value estimated in Eq. (5.3).

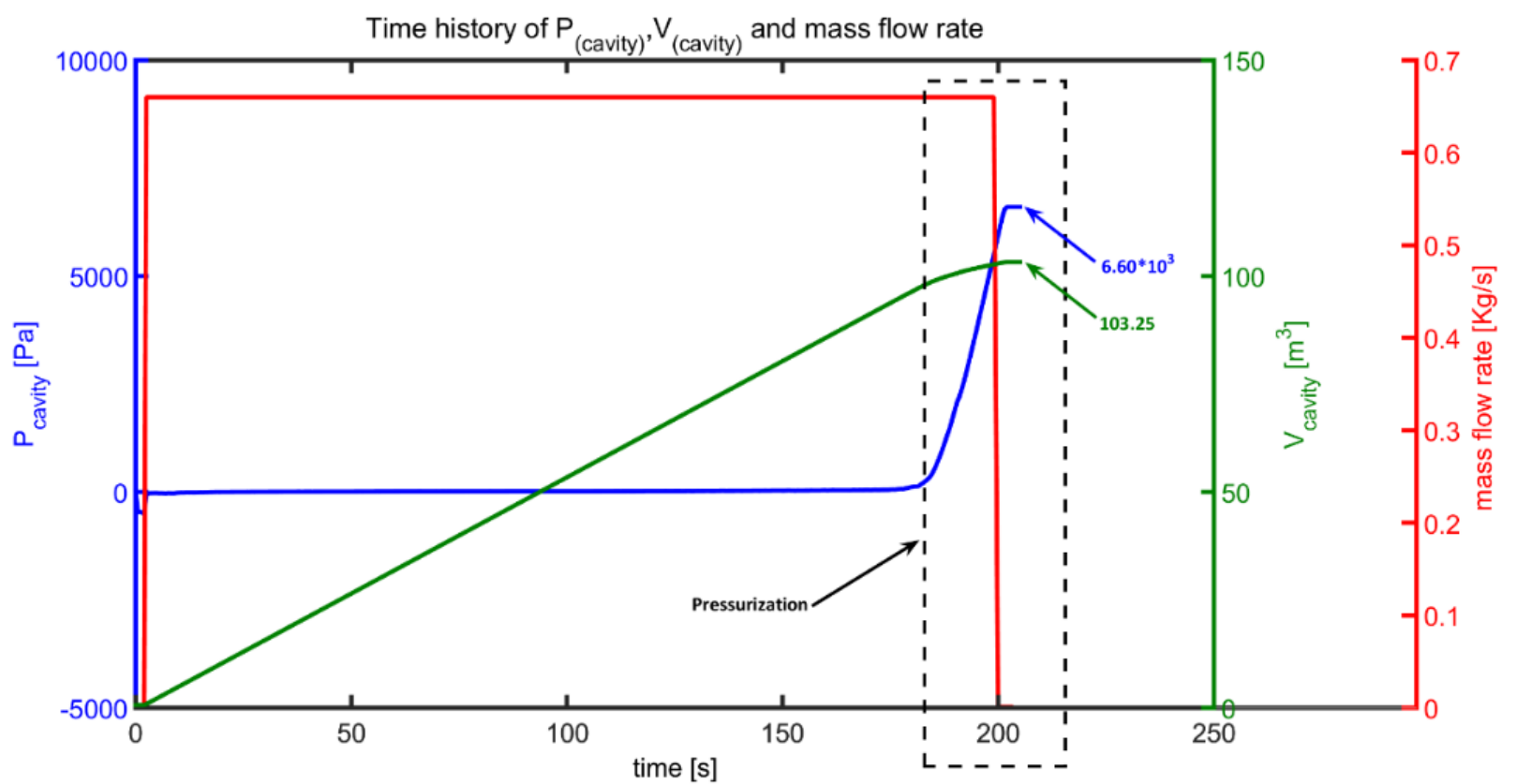

Figure 5.7. Time history of gauge pressure and internal volume for an air mass flow rate of 0.66 $\mathrm{kg} / \mathrm{sec}$.

\subsubsection{Influence of Stiffness of the Membrane during Unconfined Inflation}

A parametric study was conducted changing the value of the artificial compressive strength included in the definition of the constitutive model of the fabric material. For the different values of the compressive strength, the deformation was kept constant at a value of $\varepsilon=-0.014$, which is in the same order of magnitude of the deformation in tension for the target pressure. Six values of compressive strength were evaluated. Values ranged between $10,000 \mathrm{~Pa}$ to $500,000 \mathrm{~Pa}$ which are the range of $0.01 \%$ to $0.5 \%$ of the membrane tensile strength. The ratio between the compressive strength and the deformation produced a change in the stiffness of the membrane. The objective of trying different ratios was to reproduce a membrane behavior in the simulations that was a closer representation of what was observed in the experiments. In order to quantify the influence on the membrane flexibility produced by decreasing values of the artificial stiffness in compression, the strain energy (SE) was used as output for evaluation of the simulation results. Figure 5.8 compiles the strain energies computed for different values of the artificial 
compressive strength. Results summarized in Figure 5.8 show that the SE developed during the initial fall and unfold of the membrane (from $\mathrm{t}=0 \mathrm{sec}$ to $\mathrm{t}=10 \mathrm{sec}$ ) decreased as the compressive strength decreased, meaning that the membrane material can deform more easily for lower levels of stresses.

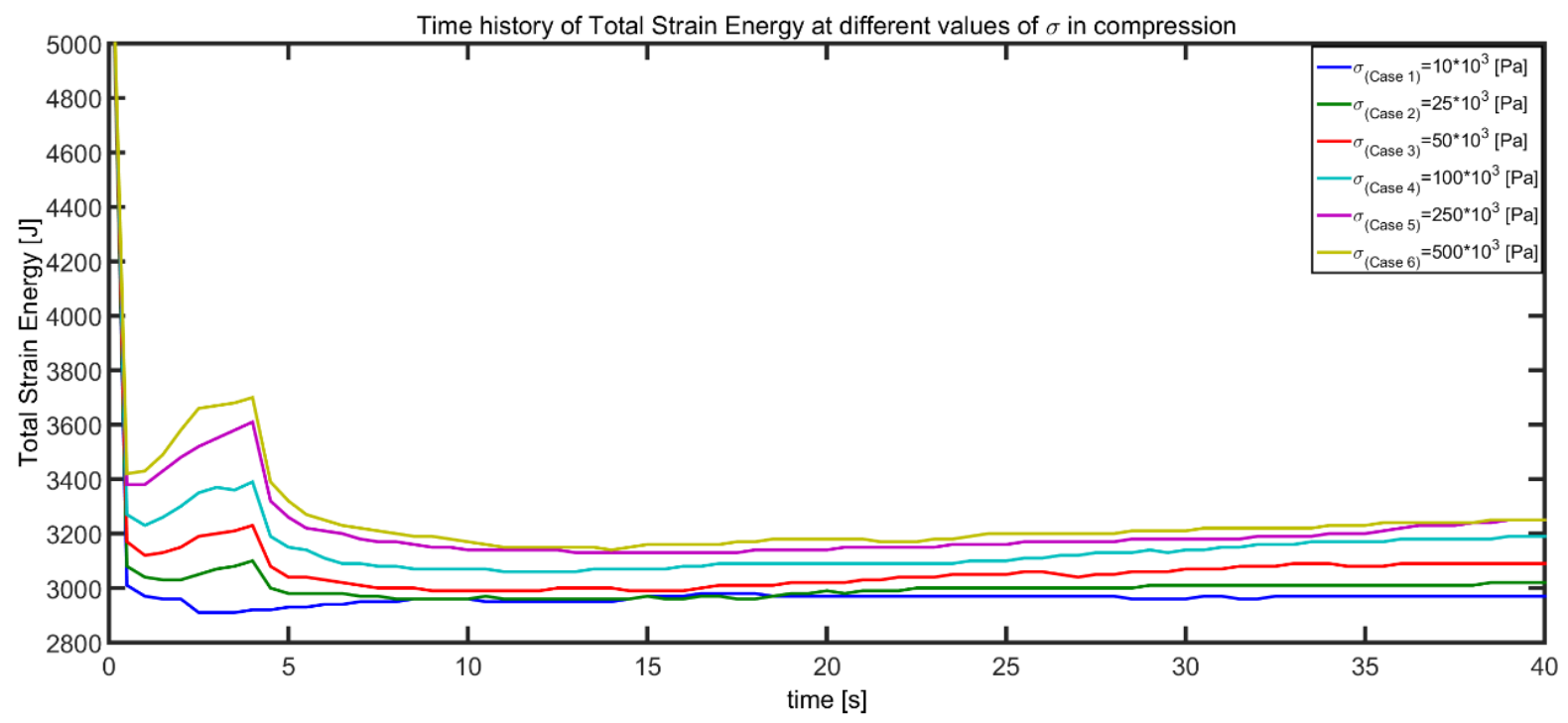

Figure 5.8. Time history of strain energy for different values of artificial compressive strength.

Figure 5.9 shows a comparison between the experimental test [7] and the deployment and inflation corresponding to Case 1, Case 3 and Case 6 taken as representative cases of the behavior of the membrane material used in the simulations. Results shown in Figure 5.9 indicate that, as expected, a decreasing compressive strength produced a more flexible behavior of the membrane during the initial unfolding and subsequent inflation. From Figure 5.9 it is possible to conclude that a value of artificial compressive strength of 10,000 $\mathrm{Pa}$ (Case 1) reproduced a membrane behavior that was closer to the membrane behavior seen in the experiments. 

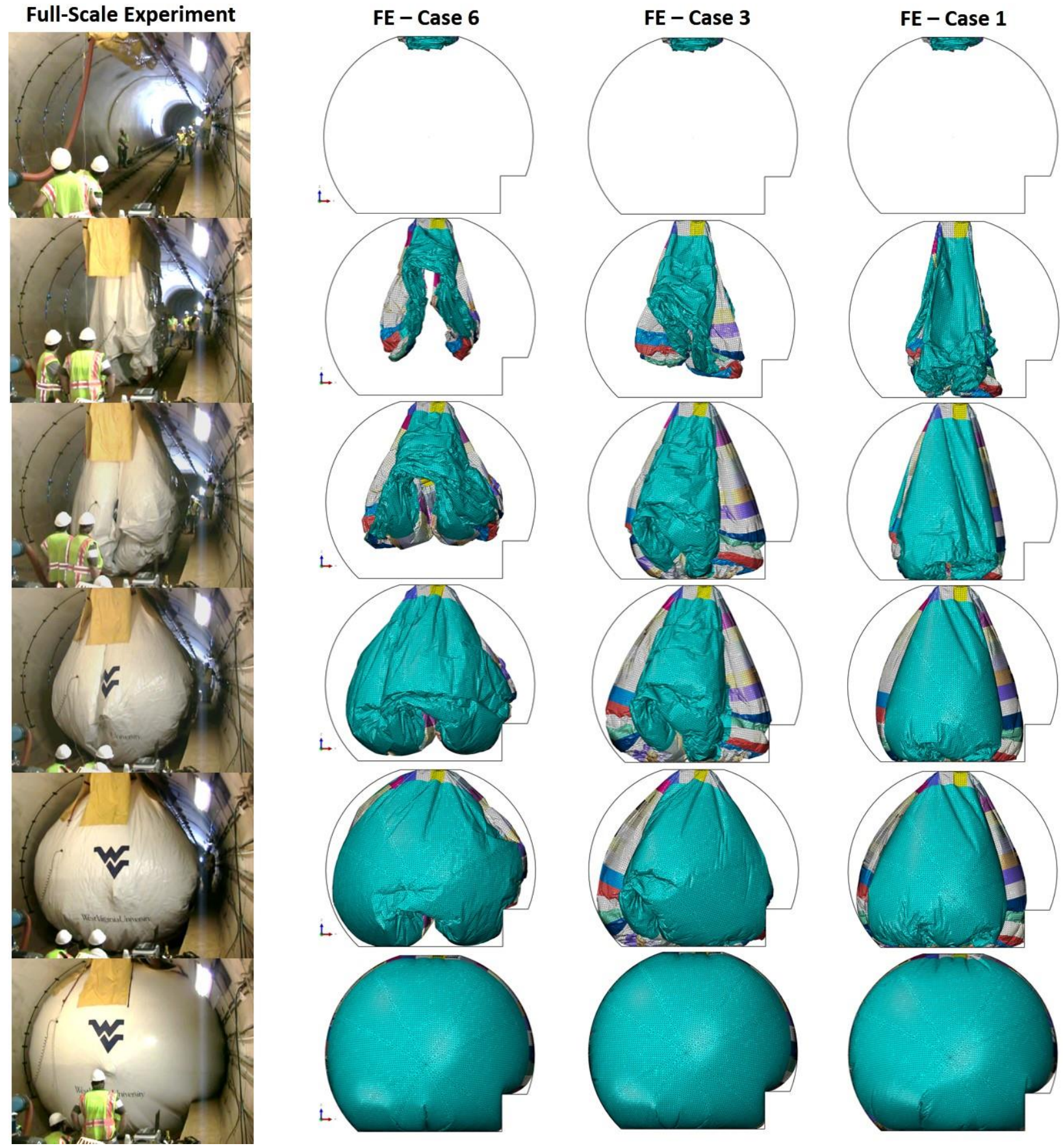

Figure 5.9. Comparison of simulation results for Cases 1,3 and 6 vs. experimental results. 


\subsection{Summary}

Three different procedures were described in this chapter: folding, the placement in the tunnel and the confined inflation. The folding sequence was implemented by using two rotating planes and two translational planes. The positions of the folding planes were selected in order to obtain a symmetric folded shape. The placement on the ceiling of the tunnel was performed in two steps by imposing translational and rotational boundary conditions to the reference node of the folded shape which was temporary defined as a rigid body.

An initial estimation of the air mass flow rate was conducted considering the new confined conditions. The confined inflation started with the folded shape positioned on the storage area on the ceiling of the tunnel, and it was completed in only one step that included the action of gravity and the activation of the inflator. This simulation of the confined inflation was used for initial understanding of the behavior of the membrane under confined inflation.

A comparison of the FE results with the experimental observations reported in [7], showed that the membrane behavior predicted by the simulation appeared to be more rigid than the behavior observed during the experiments. This problem was attributed to the artificial compressive strength adopted in the definition of the material which was initially assumed to be $0.5 \%$ of the maximum tensile strength.

A parametric study was conducted changing the value of the artificial compressive strength in order to reproduce a membrane behavior in the simulations that was closer to the experimental test. Six values of compressive strength were evaluated. The values were in the range of $0.01 \%$ to $0.5 \%$ of the tensile strength for a constant strain $\varepsilon=-0.014$. Simulation results also suggested that a value of artificial compressive strength in the range of $0.01 \%$ of the tensile strength can reproduce a membrane behavior that is closer to the membrane behavior seen in the experiments.

The global and local conformity of the FE models were evaluated as well. Results showed the presence of gaps in the corners of the tunnel. The formation of these gaps was attributed to the lack of uniform distribution of the membrane material which accumulated of tunnel floor and was not able to reach the lateral portions of the tunnel by the action of the inflation pressure. 


\section{Chapter 6. Confined Inflation - Controlled Membrane Release}

\subsection{Introduction}

This chapter presents details of an enhanced technique developed to improve the membrane behavior of the inflatable structure during the initial deployment and inflation and also to correct the lack of local conformity observed in the previous chapter. This technique developed for better control of the membrane material adopts the controlled deflation described in Chapter 4 with the addition of pre-folding steps in combination with passive restrainers. The inclusion of passive restrainers is intended to keep the position of the pre-folds during the folding procedure, and also to produce a gradual release of the membrane material during the confined inflation. An evaluation of the mechanical properties of the connector elements used to represent the passive restrainers is presented as well.

\subsection{Controlled Deflation Including Pre-folding Steps}

The controlled deflation that includes pre-folding steps is based on the same technique developed for the controlled deflation described in Section 4.2.2, but with a further improvement. In this improved process, the collapse of the membrane of the inflatable, due to the application of the gravity load, was controlled not only by the internal pneumatic pressure but also by applying additional displacement boundary conditions to specific lines and portions of the membrane to form initial pre-folds.

Two cases of controlled deflation including pre-folds were simulated: the first one, Case A, included only one pre-fold, and the second one, Case B, included two pre-folds. Case A was developed to simulate the technique of the controlled release of the membrane similar to the one implemented in the experiments reported in [7]. Case B was created to show the potential of this new technique and to emphasize the possibility of achieving a higher level of local conformity of the membrane for different tunnel profiles.

The position of the initial pre-folds was dictated by the position of folding surfaces of the membrane that did not conform to specific locations (corners) in the tunnel profile at the end of the inflation as shown in Figure 5.6. In the simulation of Cases $\mathrm{A}$ and $\mathrm{B}$, the controlled deflation with pre-folding steps was performed using the same slope used in the controlled deflation described in Section 4.2.2. Also, as described in Section 4.2.2, the simulation was stopped after four to eight iterations to capture the coordinates of the resultant shape with Abaqus/CAE and then checked for penetrations and intersections of elements in Hypermesh. Once the verification was completed, the resultant deformed shape was reused for continuing the simulation using the same initial conditions of pressure $\left(P_{\text {internal }}=P_{\text {gravity }}\right)$ and the same decreasing ramp. 


\subsubsection{Case A}

The initial shape used at the beginning of the simulation was the initial nominal shape of the inflatable structure shown in Figure 3.4. The initial single pre-fold, as mention above, was chosen considering the position of the folding surfaces (colored bands in Figure 3.4) necessary to cover the critical corners of the tunnel. To achieve a symmetric flat deflated shape, another pre-fold was created on the opposite side of the first pre-fold to get an equal distribution of the membrane material as shown in Figure 6.1. In Figure 6.2(a), the highlighted lines show the nodes where the translation boundary conditions were applied. This process was repeated eight times (8 steps), and each step was denominated cleaning process. Each step was performed using a mass scale factor of 10, a mass proportional damping factor of $\alpha=0.4$ and no contact penalty factor. Contact interactions and friction were defined between the membrane of the inflatable and the base $(\mu=0.40)$ and between the membrane of the inflatable and itself $(\mu=0.20)$.

In Figure 6.2(a), the nodes on the center lines (CL) were set to move along the $\mathrm{X}$-axis and along the $\mathrm{Z}$ axis, whereas the nodes on the bottom and the top lines (BL and TL, respectively) were set to move only along the $\mathrm{Z}$ axis, and all the nodes on the lines were constrained to move along the $\mathrm{Y}$-axis. A horizontal displacement of $|1.6| \mathrm{m}$ along the $\mathrm{X}$ axis was imposed as translation boundary condition to the center lines as shown in Figures 6.2(b) and 6.2(c). The simultaneous displacement of the nodes on the center lines along the $\mathrm{X}$ axis and the decrease of the pneumatic pressure produced the translation along the $\mathrm{Z}$ axis of the nodes on the bottom and on the top lines until they were close to each other as shown in Figure 6.2(d). From that moment on, all the boundary conditions applied to the nodes on the center, bottom and top lines were deactivated. Once the two initial pre-folds were formed, as illustrated in Figure 6.2(e), equally spaced nodes located at the bottom and the top lines were linked with connector elements to maintain the lines close to each other to maintain the shape of the folding surfaces generated by the pre-folds.

A displacement along the $\mathrm{X}$ axis of $|0.36| \mathrm{m}$ was imposed as translational boundary condition to the nodes on the highlighted lines to stretch the membrane, as shown in Figure 6.2(f). After reaching the final shape shown in Figure 6.2(g), the nodes on the highlighted lines were set to move only along the $\mathrm{Z}$ axis and the final shape is shown in Figure 6.2(h). In order to reach a flatter shape, instead of applying a gravity load, as done in the controlled deflation, a vacuum pressure of $700 \mathrm{~Pa}$ was applied as boundary condition in order to be more realistic and feasible experimentally. A contact penalty factor of 20 was implemented in this last step to avoid intersection and penetration of the elements. The final flattened shape is shown in Figure 6.2(i). 

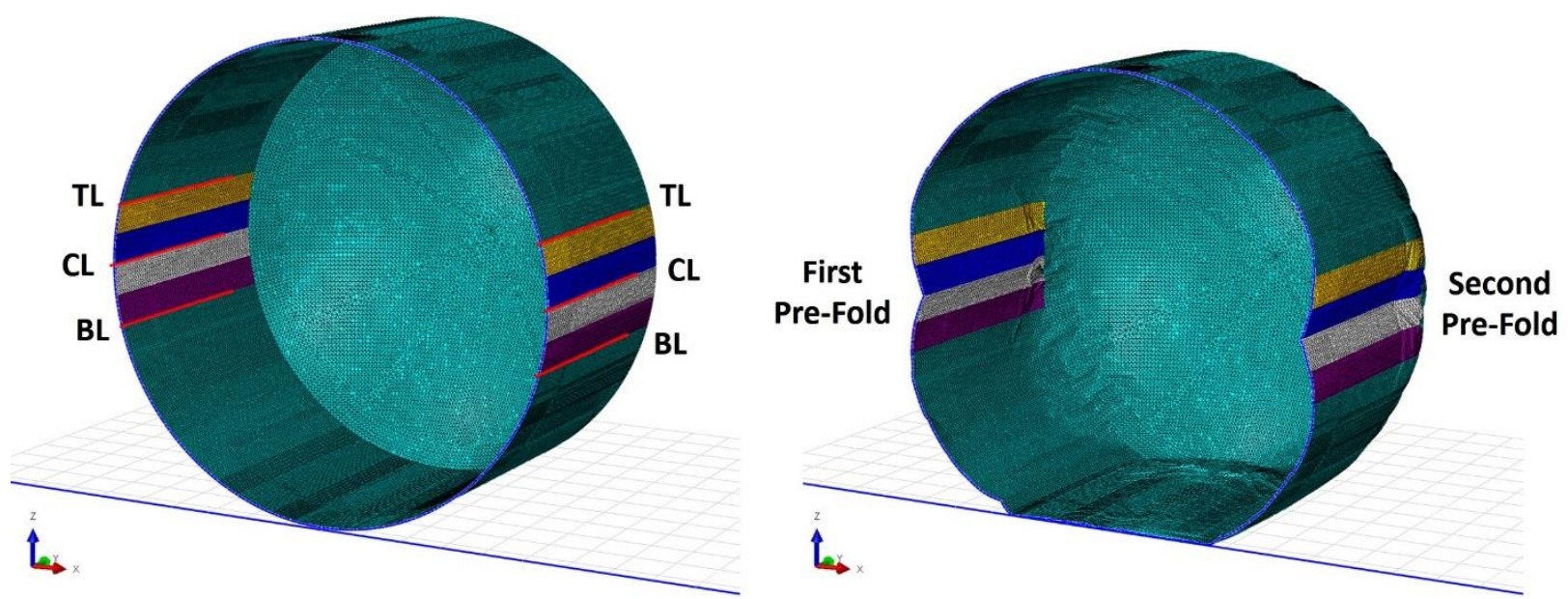

Figure 6.1. Controlled deflation, reference lines and position of pre-folds, Case A.

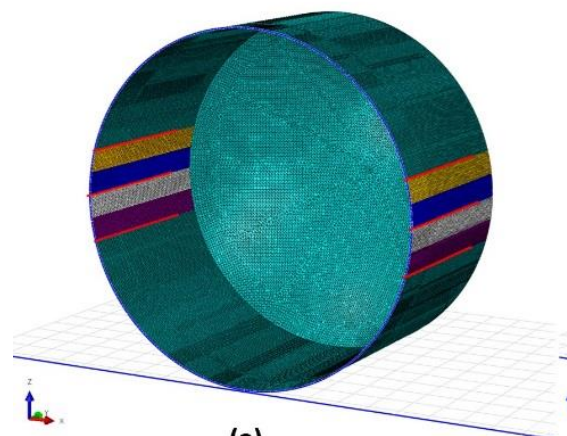

(a)

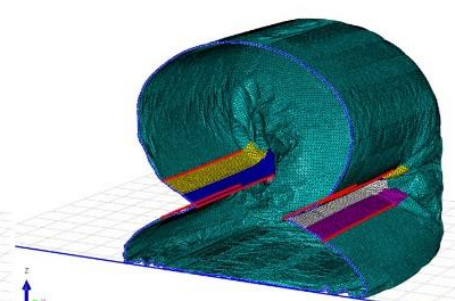

(b)

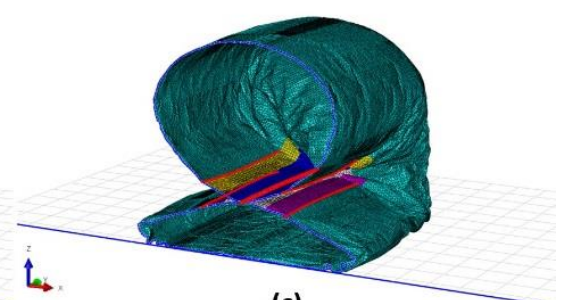

(c)

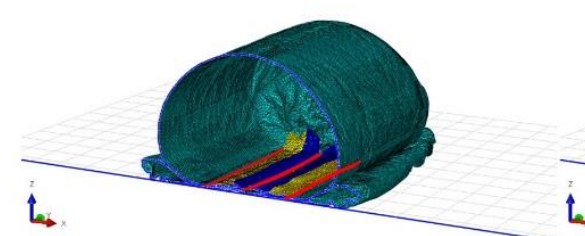

(d)

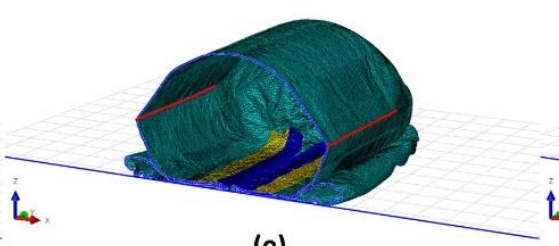

(e)

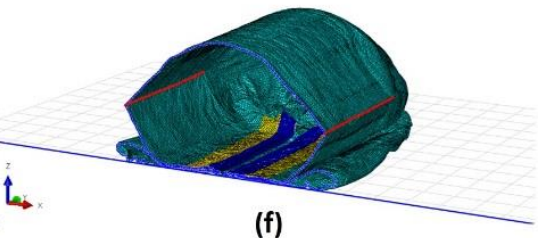

(f)

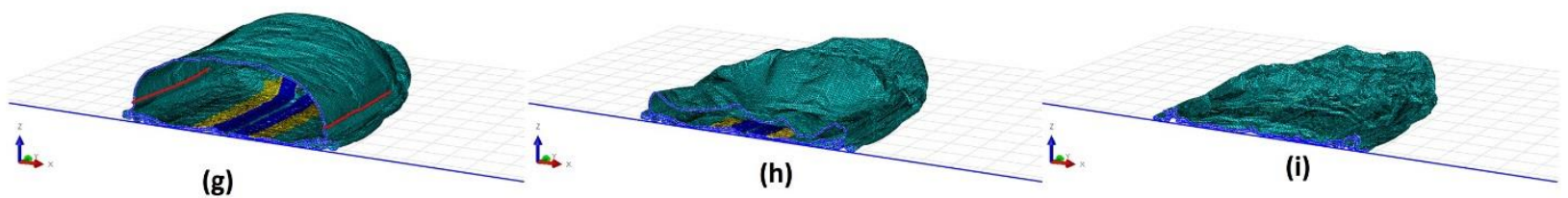

Figure 6.2. Sequence of controlled deflation with pre-folding steps Case A. 


\subsubsection{Case B}

The initial shape used at the beginning of the simulation of Case B was the shape obtained at the end of the fifth cleaning step of Case A, as shown in 6.4(a). Case B required three iterations, and it was performed using a mass scale factor of 10, a mass proportional damping factor of $\alpha=0.4$ and no contact penalty factor. A third pre-fold was introduced considering the initial position of the folding surfaces that were closer to the lower right corner of the tunnel profile. The position of the third pre-fold and the folding lines are illustrated in Figure 6.3. As illustrated in Figure 6.4(b), displacement boundary conditions were imposed to the nodes on the highlighted lines to stretch the membrane in preparation for creation of the third prefold. The third pre-fold was created by applying translational boundary conditions to the nodes on the highlighted lines illustrated in Figure 6.4(c). Once the third pre-fold was formed, equally spaced nodes on the closer lines of the fold were linked with connector elements as done in the Case A. Also, as in Case A, and in order to reach a flatter shape, a vacuum pressure of $700 \mathrm{~Pa}$ was applied as boundary condition, as illustrated in Figure 6.4(d).

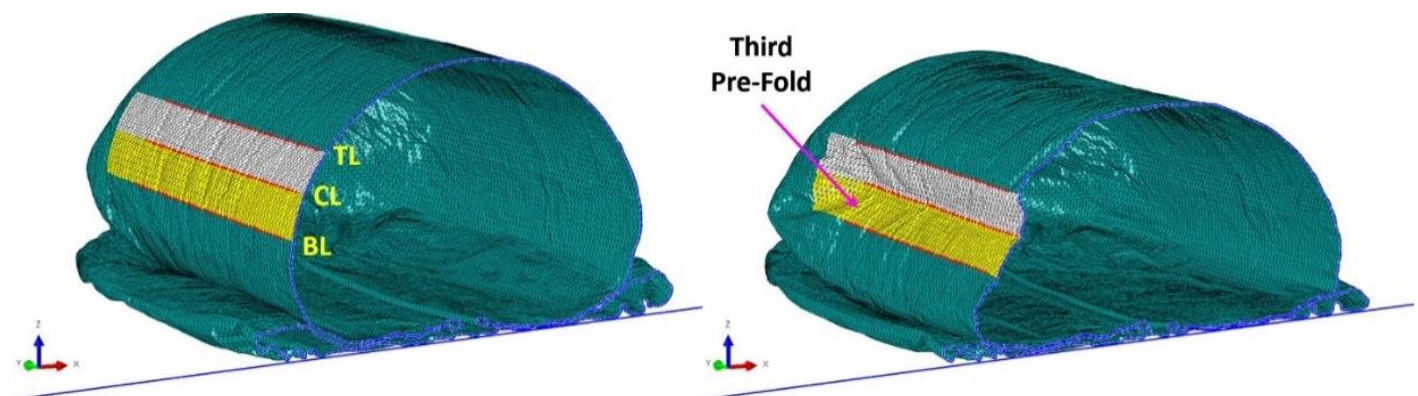

Figure 6.3. Controlled deflation, reference lines and position of pre-folds, Case B.

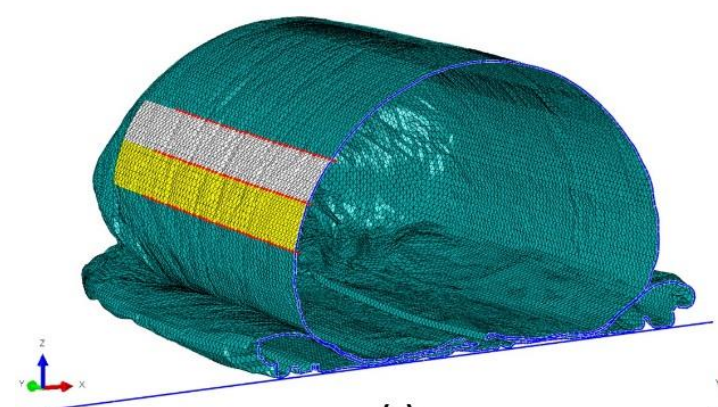

(a)

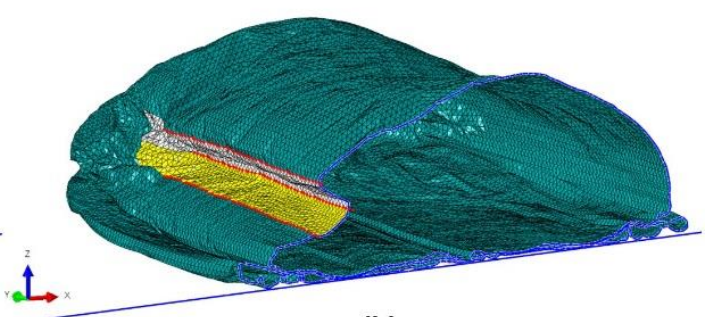

(b)

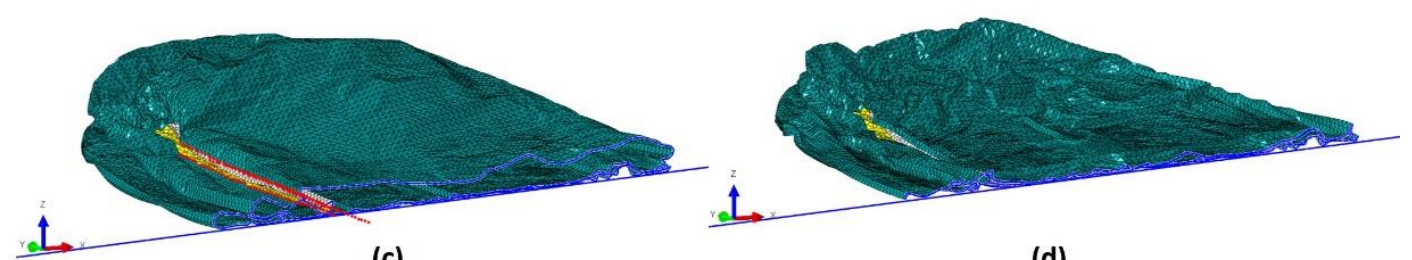

(c)

(d)

Figure 6.4. Sequence of controlled deflation with pre-folding steps Case B. 


\subsection{Design of Passive Restrainers}

A controlled release of the membrane material was implemented in the experiments reported in [7]. In these experiments, it was observed that during the inflation process the passive restrainers holding the membrane material of the inflatable broke around the 180th second of the inflation.

The pressure history obtained from the simulation of confined inflation without control of the membrane (Figure 5.7) provided the value of the gauge pressure at the 180th second that was $180 \mathrm{~Pa}$. This value of pressure is the pressure that the connectors will have to take at breakage $\left(P_{\text {conn }}\right)$. A preliminary estimation of the magnitude of the force that each passive restrainer will have to take before breaking to release the membrane is based on the hoop stress on the cylindrical portion of the inflatable given by:

$$
\sigma_{\text {hoop }} t=P_{\text {conn }} R=451.8 \frac{N}{m}
$$

where $\mathrm{t}$ is the thickness of the membrane, $P_{\text {conn }}$ is the pressure necessary to break the passive restrainers, and $\mathrm{R}$ is the radius of the cross-section of the cylindrical of the inflatable structure at the 180th second of the inflation. Looking at the inflate shape in Figure 5.6(a), the value of $\mathrm{R}$ was initially assumed to be approximately equal to the radius of the tunnel. This initial estimation of $\mathrm{R}$ is slightly overestimated but close enough to obtain the range of force that the restrainers would have to take. The value obtained in Eq. (6.1) is the hoop force on the cylinder per unit of length. The total force acting in the hoop direction of the cylinder is the product between the unit force of Eq. (6.1) and the nominal length of the cylindrical portion of the inflatable and it is equal to $1652.2 \mathrm{~N}$. This total force is the active force in the hoop direction that has to be equal to the total reaction force carried out by all the passive restrainers before their breakage. In the experiments reported in [7], a total of six passive restrainers were installed to control the release of the membrane material on the cylindrical portion of the inflatable. In this work, the same number was adopted to simulate the connectors installed along the first pre-fold shown in Figure 6.2(d). By dividing the total force of $1652.2 \mathrm{~N}$ into six passive restrainers, the force that each one will take individually before their breakage at the pressure $P_{\text {conn }}$ is equal to $275 \mathrm{~N}$.

Considering that the passive restrainers are typically manufactured from materials that are commercially available, such as synthetic filaments and other similar materials, this work adopted values of individual breakage force in the range of $267 \mathrm{~N}$ (60 lbf) to $311 \mathrm{~N}$ (70 lbf) [38]. Since the force was calculated using an overestimated radius, a passive restrainer with a nominal strength of $267 \mathrm{~N}$ was adopted for the simulations. 


\subsection{Folding Process}

The flattened shapes obtained at the end of the controlled deflation with pre-folding steps corresponding to Case A and Case B were folded imposing translational and rotational boundary conditions to the folding planes FP1 and FP2 illustrated in Figure 6.5. The folding procedure was the same for both cases. Six nodes on the top and the bottom edges of each pre-folding lines were restrained using connector elements to avoid sliding of the membrane material during the folding process that could cause excessive distortion of the pre-folds. The folding process was performed using an MSF $=100$, a mass proportional damping factor $\alpha=0.4$ and no contact penalty factor. The reduction in the number of the planes need to perform the folding process was due to the presence of internal pre-folds that reduced the footprint of the flattened shape. The dimensions of the folding planes were adjusted according to the dimensions of the completely deflated shape.

The folding sequence illustrated in Figure 6.5 was completed in five simulation steps to achieve the final folded shape. The position of the folding planes was selected to perform a symmetric folding sequence. In Figure 6.5, the colors of the folds correspond to the colors of the folding planes that were used to perform the specific folding step.

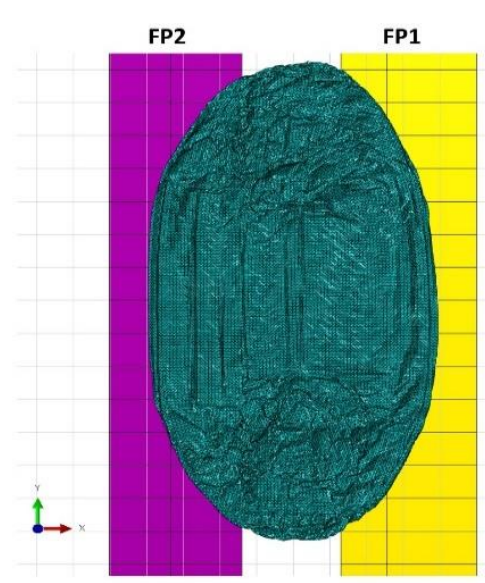

(a)

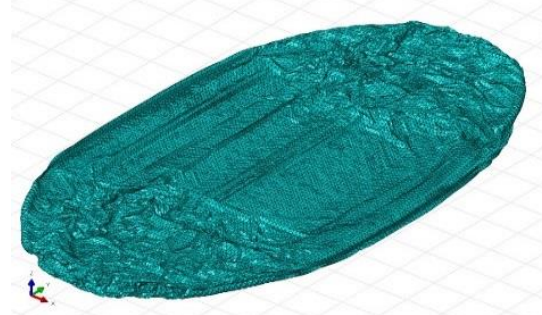

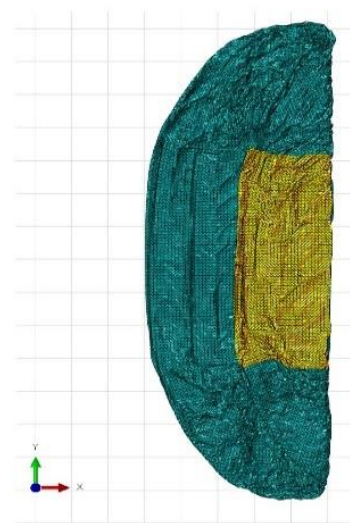

(b)

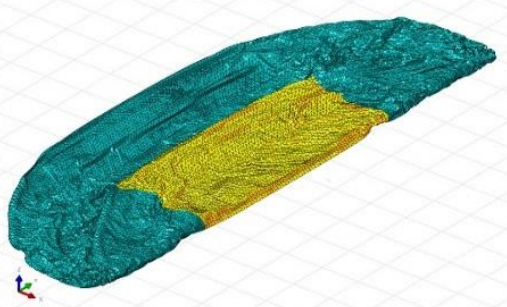

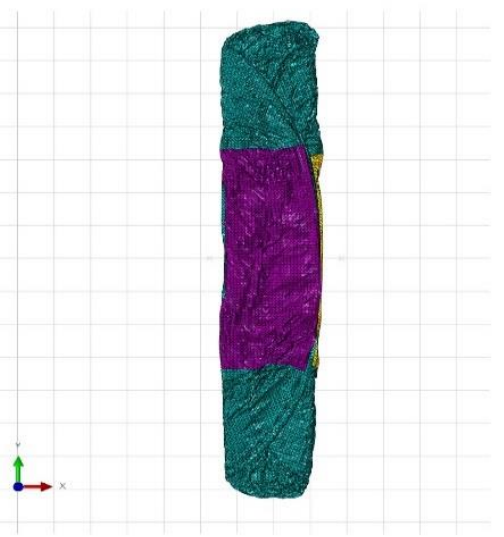

(c)

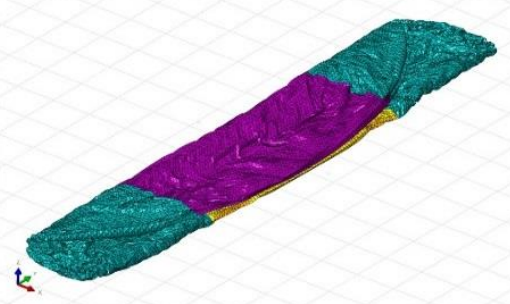

Figure 6.5. Folding sequence, main folding steps, top and isometric views. 
The sequence of steps performed to reach the folded shape is illustrated in Figure 6.6 include:

1) In the first simulation step shown in Figure 6.6(a), the inflatable was initially flattened by the action of gravity applied to all membrane elements along the Z-axis. After this initial step, gravity was active for the total duration of the folding process. Contact interactions and friction were defined between the membrane of the inflatable and the base $(\mu=0.50)$, between the membrane of the inflatable and itself $(\mu=0.50)$ and between the inflatable structure and the folding planes $(\mu=0.50)$. No contact interactions were assigned between the rigid bodies (base and folding planes).

2) In the second simulation step shown in Figure 6.6(b), the folding sequence started by imposing boundary conditions to the reference nodes of the folding planes acting as rigid bodies. The reference node of the folding plane FP1 was constrained to translate vertically along the Z-axis $\left(U_{3}=0.10 \mathrm{~m}\right)$.

3) In the third simulation step shown in Figure 6.6(c), the reference node of the folding plane FP1 was set to rotate along the $\mathrm{Y}$ axis $\left(U_{5}=180^{\circ}\right)$. At the end of this step the first fold was completed and it is shown in Figure $6.6(d)$.

4) The second fold was performed by the folding plane FP2 which followed the same steps of FP1 but with its rotation performed in opposite directions to achieve the second fold symmetric to the one described in steps 2 and 3. This sequence is shown in Figures 6.6(e) to 6.6(h). At the end of this sequence, the second fold was completed as illustrated in Figure 6.6(h).

5) The resultant folded shape shown in Figure 6.6(h) was verified for penetrations and intersections of elements as explained in Section 5.2. The verified mesh was then placed in the tunnel using the same procedure described in Section 5.3.

Before proceeding to describe the confined inflation with the implementation of the controlled release of the membrane, it is important to underline the remarkable improvements achieved in terms of the final folded shape, in the reduction of simulation steps and, also in terms of the flatness of the deflated shape reached at the end of the controlled deflation compared with the simulation without the implementation of pre-folds.

Figure 6.7 compares two folded shapes positioned on the ceiling of the tunnel. Figure 6.7(a) shows the folded shape obtained without the inclusion of pre-folds (Case 0), whereas Figure 6.7(b) shows the folded shape obtained using the pre-folds (Case A and B). Comparing Figure 6.7(a) and Figure 6.7(b), it is possible to see a significant reduction of the overall folded volume and a more uniform distribution of the membrane material along the total longitudinal length of the inflatable. The reduction of the thickness of the folded shape is also indicated in Figure 6.7. For Case 0, w(a) was $0.32 \mathrm{~m}$ and for Case A and B, w(b) was $0.18 \mathrm{~m}$, which is almost half of Case 0. 


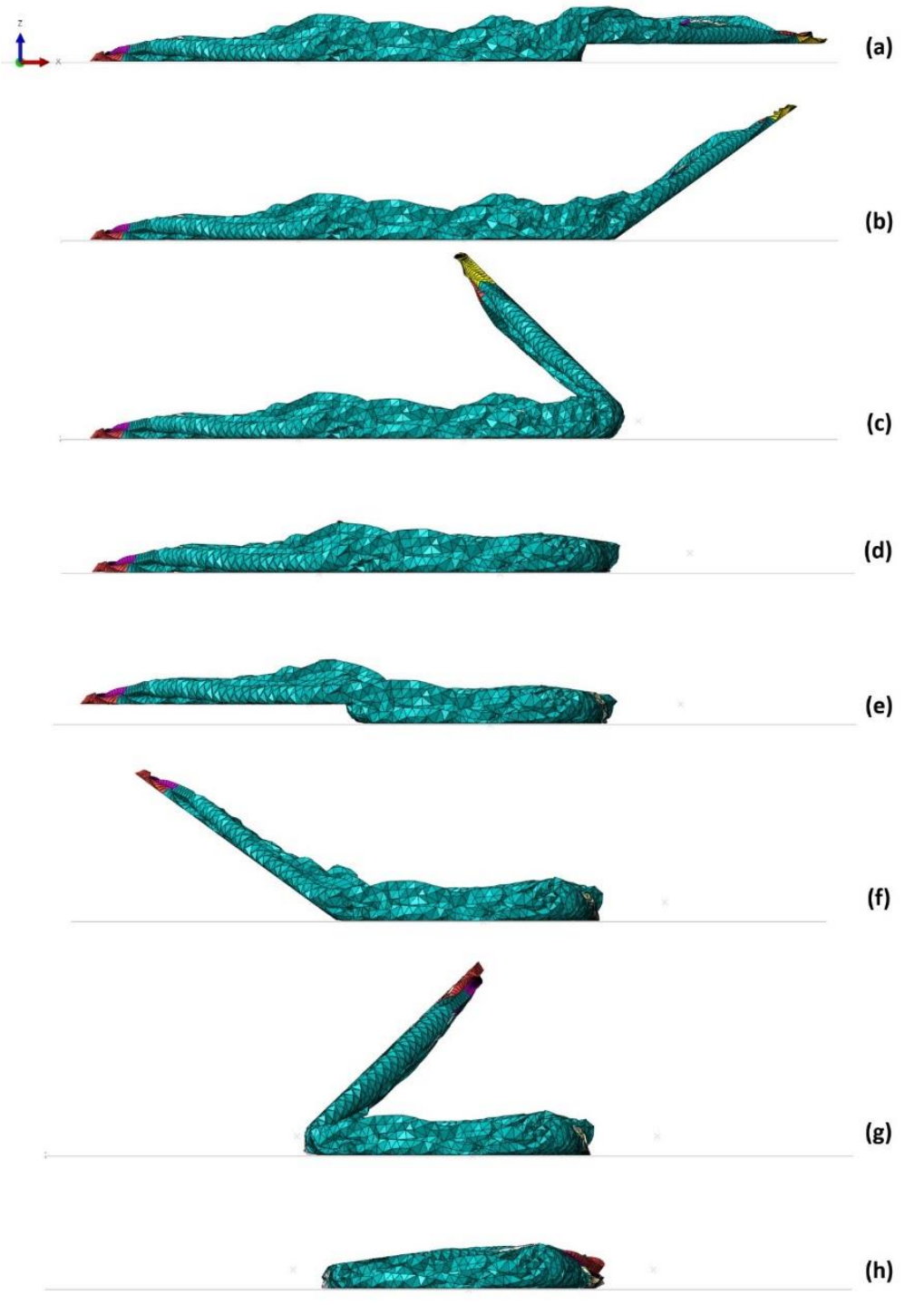

Figure 6.6. Lateral view of the folding sequence corresponding to Cases A and B.

Moreover, the controlled deflation including pre-folds implemented in Cases A and B was performed in eight simulation steps, which is less than half of the simulation steps need to complete the controlled deflation without pre-folds (Case 0). The controlled deflation implemented in Case 0 described in Section 4.2.2 required 19 simulation steps. Considering the technique used to reach a flatter shape at the end of the controlled deflation, the implementation of vacuum in the last simulation step of the controlled deflation produced an even flatter shape in Cases A and B. Although a similar flat shape was achieved in Case 0 using ten times the gravity, the implementation of vacuum is more feasible to be executed experimentally. 
Furthermore, the folding procedure implemented in Cases A and B was performed in five simulation steps, which were less than half of the simulation steps implemented in Case 0 (13 simulation steps).
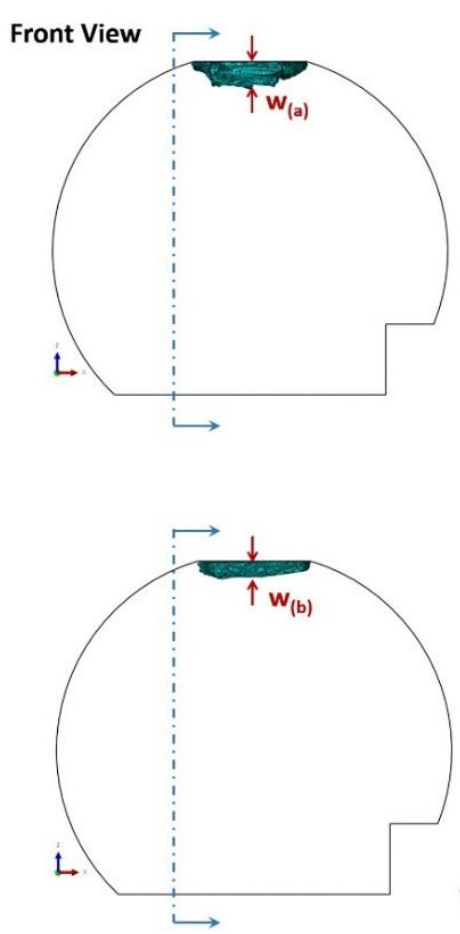

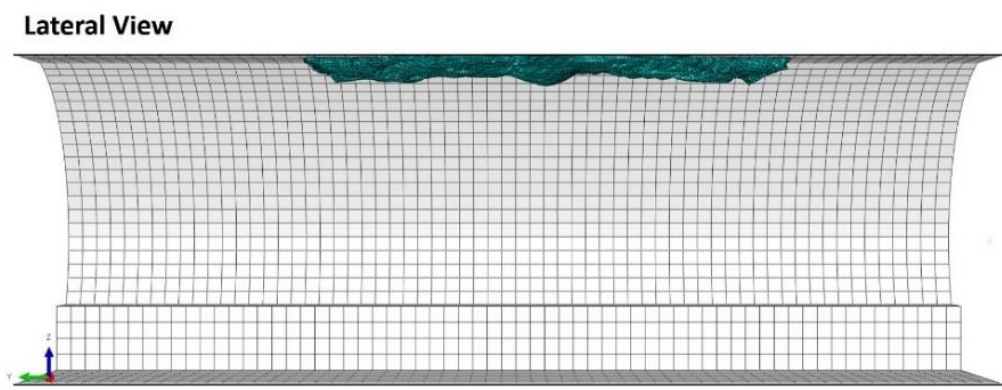

(a)

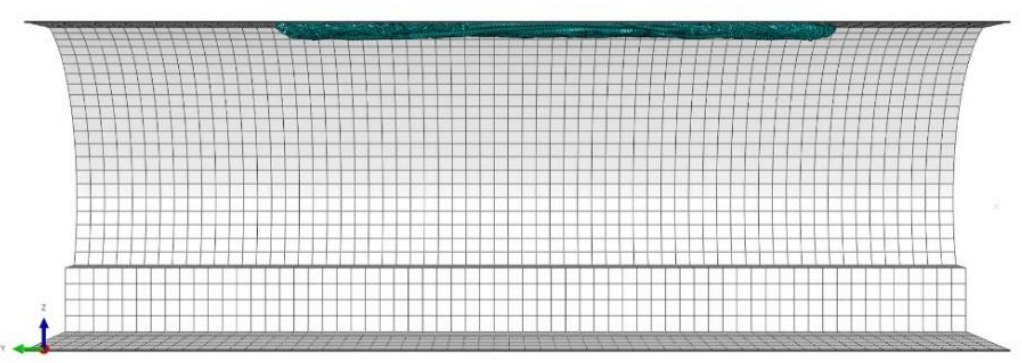

(b)

Figure 6.7. (a) Folded shape without pre-folds (Case 0); (b) Folded shape including pre-folds (Cases $A$ and B).

\subsection{Confined Inflation with Controlled Release of Membrane}

The simulation of the deployment and inflation implementing a controlled release of the membrane material was similar to the process described in Section 5.5 except for the presence of the passive restrainers modeled with connector elements. Case A included only one pre-fold to control the release of the membrane, and Case B included two pre-folds for controlling the release of the membrane.

In both simulation cases, the confined inflation was performed using an MSF of 100, a mass proportional damping factor $\alpha=0.2$ and a penalty factor of 10 . Contact interactions and friction were defined between the membrane of the inflatable and the tunnel $(\mu=0.40)$ and between the membrane of the inflatable and itself $(\mu=0.20)$. An artificial compressive strength of $0.02 \%$ of the membrane tensile strength was included in the definition of the constitutive model of the fabric material. The total simulation time including the initial deployment followed by the inflation sequence was set to take place in 200 seconds plus 5 additional seconds for deactivation of the inflator and final pressure stabilization. The initial inflator defined in Section 5.4 with an air mass flow rate of $0.66 \frac{\mathrm{kg}}{\mathrm{sec}}$, was slightly increased to $0.67 \frac{\mathrm{kg}}{\mathrm{sec}}$ to account 
for the drop of internal pressure due to the breakage of the connectors during the inflation process. The sequence of deployment and inflation for Cases A and B are shown in Figure 6.8 and compared with the experimental tests reported in [7].
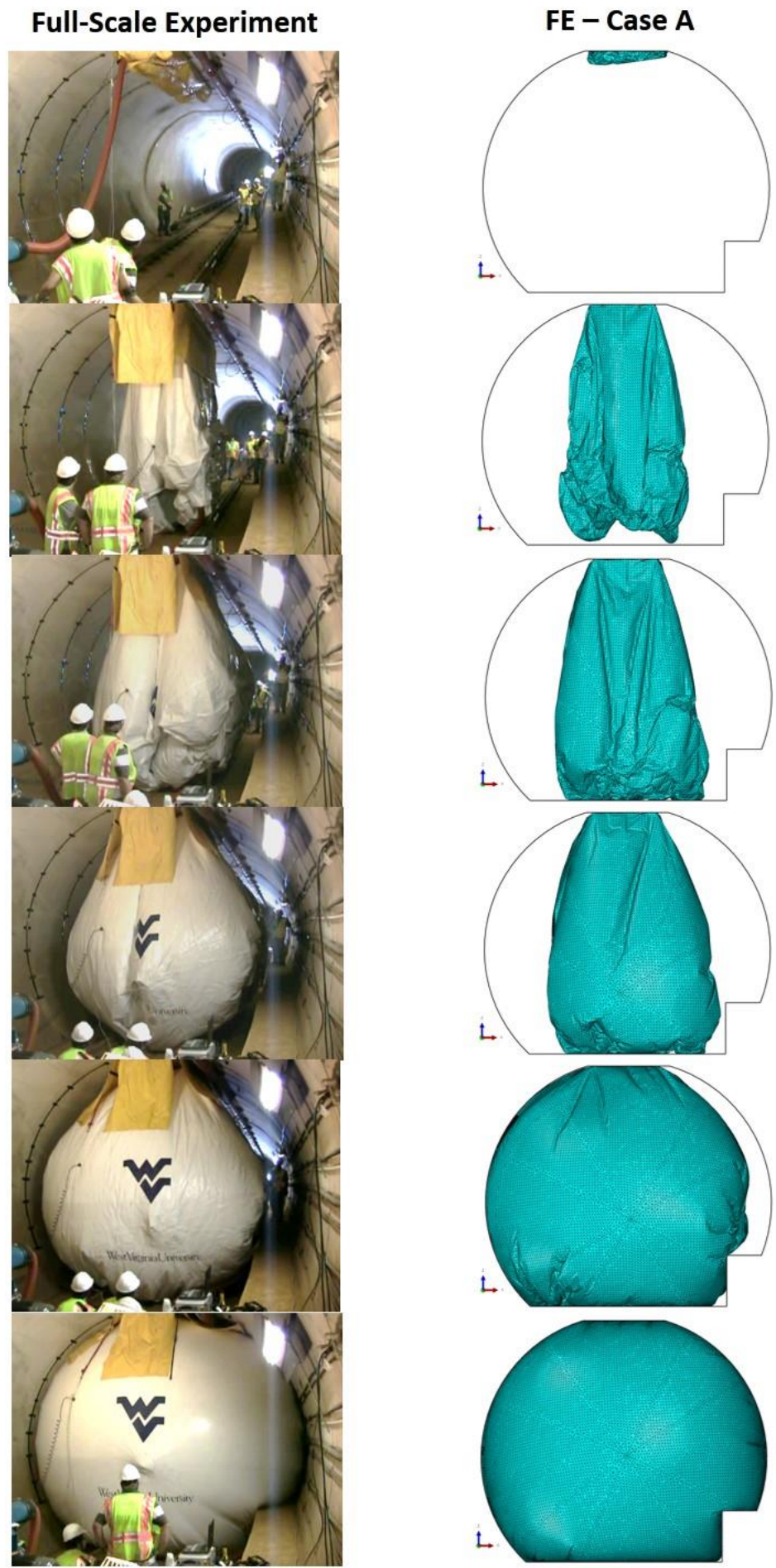

(a)

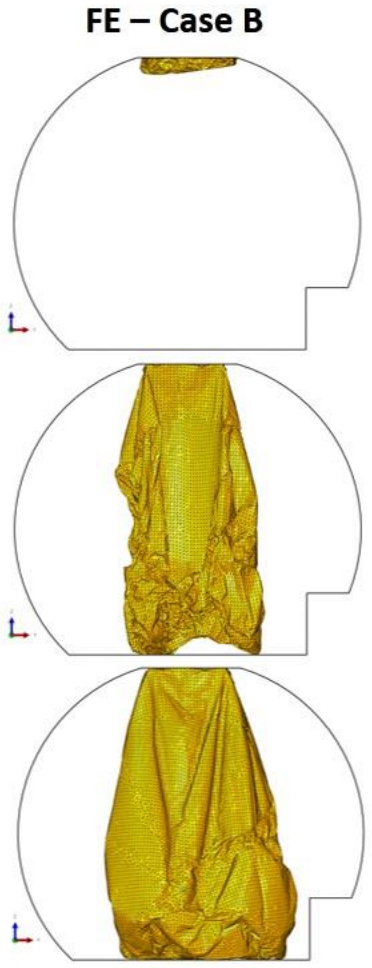

(c)

(b)

(d)

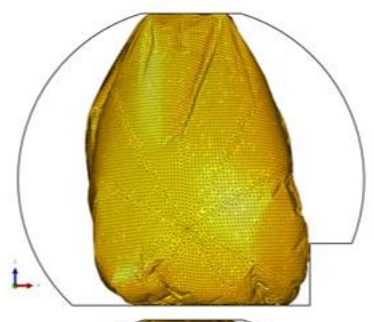

(e)

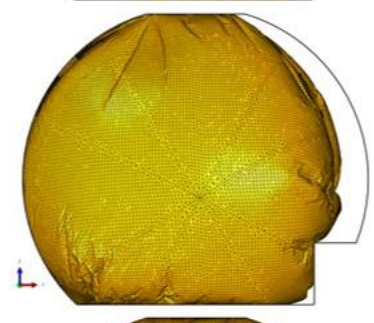

(f)

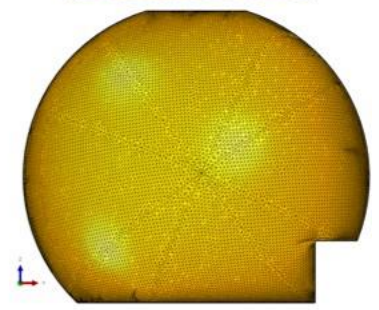

Figure 6.8. Comparison of simulation results for Case A and Case B vs. experimental results. 

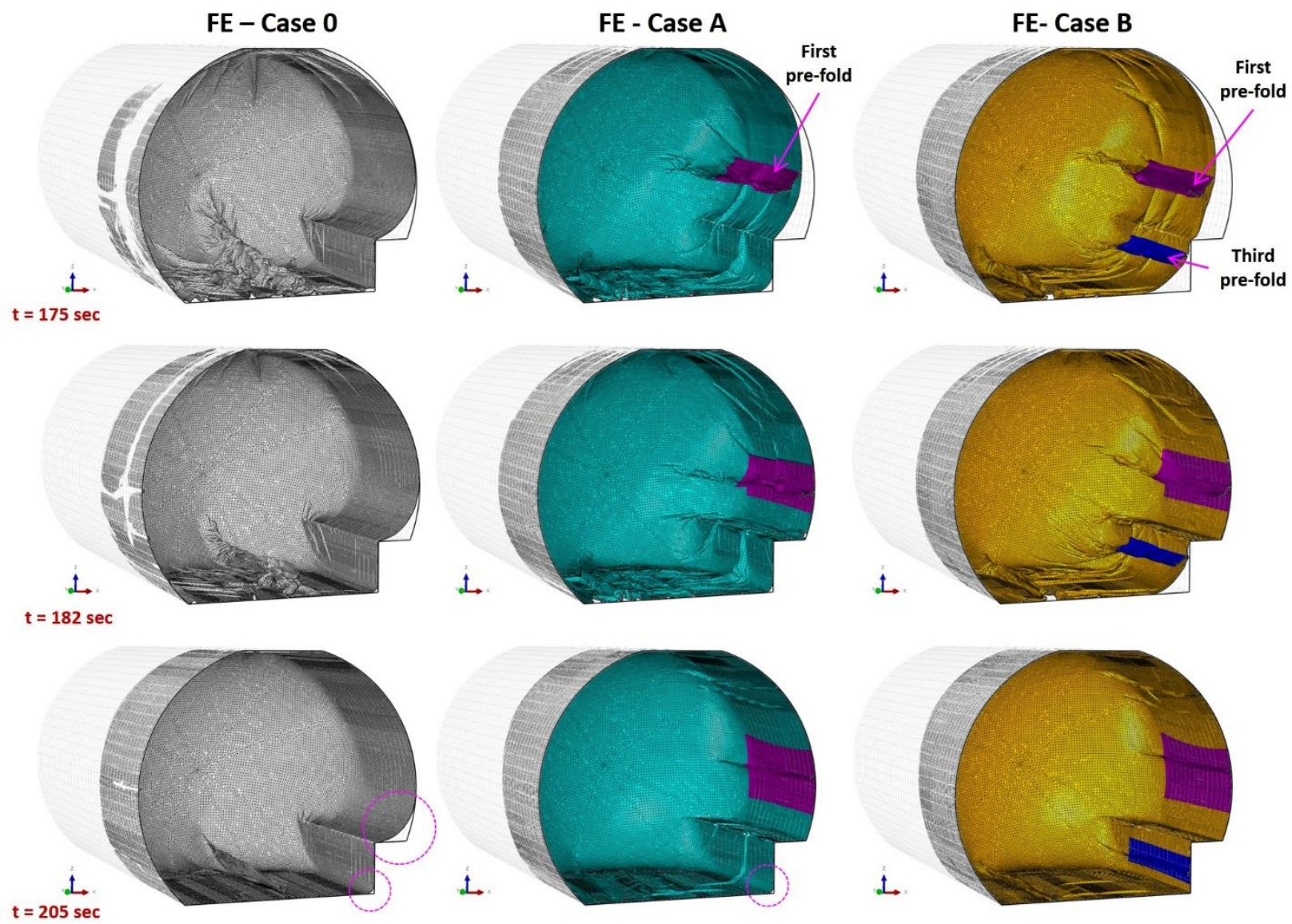

Figure 6.9. Release of the membrane. Comparison of simulation results for Case $0, A$ and Case B.

Figure 6.8 illustrates the following aspects of the membrane behavior during deployment and inflation:

- In both Cases A and B, the behavior of the membrane modeled using an artificial compressive strength with a value of $0.02 \%$ of the membrane tensile strength reproduced qualitatively an unfolding and expansion of the inflatable similar to behavior seen in the experiments.

- Regarding global conformity of the inflatable to the tunnel perimeter, the inflated shapes seen in Cases $\mathrm{A}$ and $\mathrm{B}$ at the end of the simulations were similar to the shape observed in the experiments.

- For Case A, and in terms of local conformity of the inflatable to the corners of the tunnel perimeter, the simulation results show that inflatable was able to conform to the upper right corner, but it was not able to completely fill the lower right corner of the tunnel as shown in Figure 6.8(f). Comparing Case 0 and Case A illustrated in Figure 6.9, it is possible to observe an improvement in the conformity on the upper right corner of the tunnel. This improvement is attributed to the inclusion of the pre-fold which released upon breakage of the passive restrainers at the end of the inflation. 
- For Case B, and also in terms of local conformity, Figure 6.9 shows a remarkable improvement in the local conformity in both corners of the tunnel. This improvement is attributed to the inclusion of two pre-folds and the release of the membrane contained in the pre-folds upon nearly simultaneous breakage of the passive restrainers at the end of the inflation. These results demonstrate how the control of the membrane during the controlled deflation, folding, and inflation with controlled release of the membrane material contributes to reach a higher level of local conformity of the inflatable to the tunnel perimeter.

In the simulation corresponding to Case B, a second set of passive restrainers were used in the third prefold shown in Figure 6.4(c). The number of passive restrainers used in the third pre-fold was calculated taking into account the radius of curvature (R2) of the inflatable in the proximity of the lower right corner of the tunnel before the breakage of the connectors in Case A, as illustrated in Figure 6.10. From Figure 6.10 , it is possible to see that the radius of curvature on the lower right corner of the tunnel is about half of the radius of the inflatable (R1) at the onset of the breakage of the passive restrainers of the first pre-fold. Since the purpose is to use the same type of passive restrainers used in Case A and also considering Eq. (6.1), a total of three passive restrainers were implemented along the cylindrical portion of the inflatable at the position of the third pre-fold.

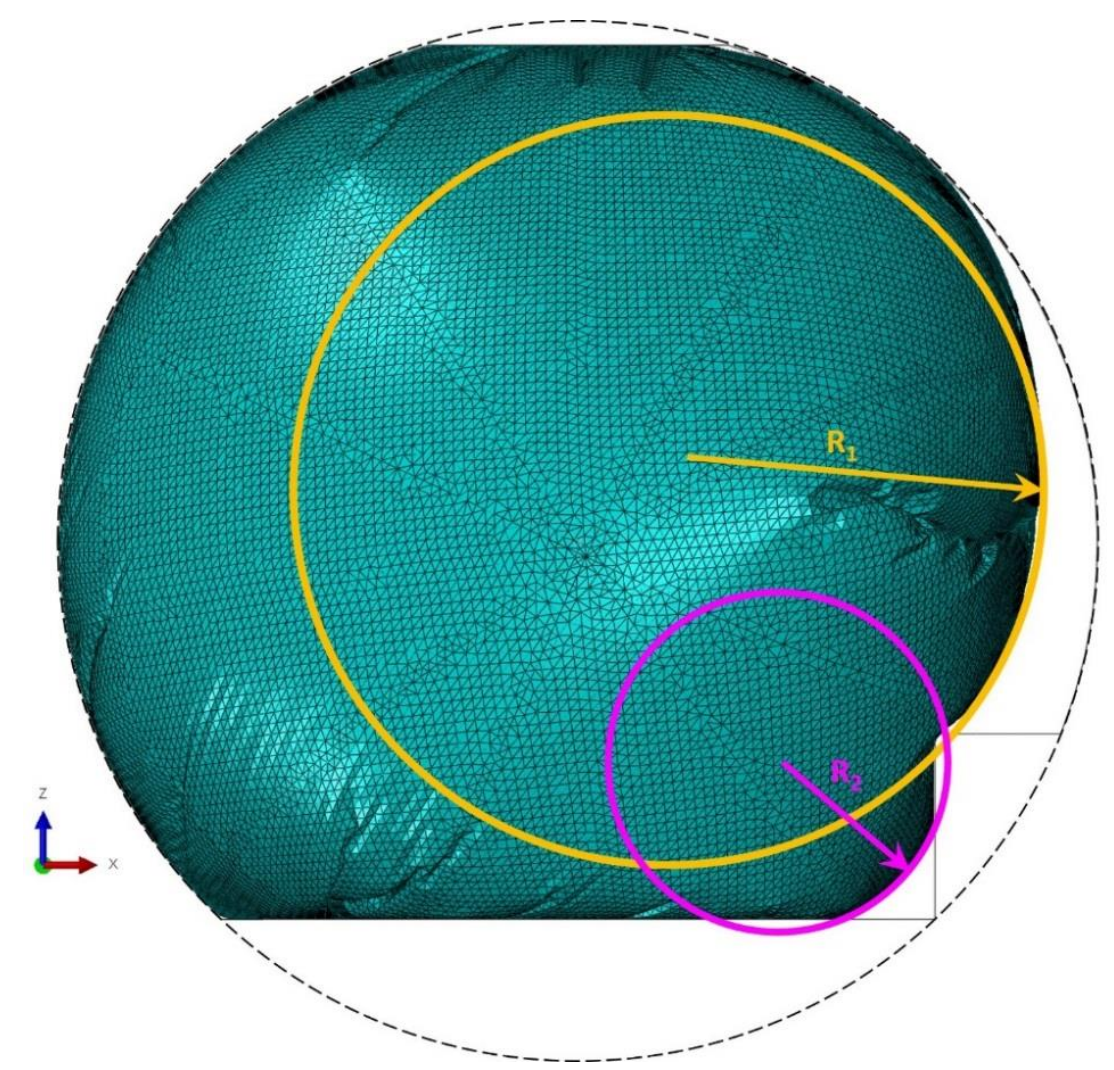

Figure 6.10. Radii of curvature of inflatable at the onset of failure of passive restrainers. 
From a thermodynamic point of view, and in order to understand the effects of the inclusion of passive restrainers, the time history of the internal pressure, the internal volume and the failure status of the passive restrainers (where 0 corresponds to no breakage, and 1 corresponds to breakage), are plotted in Figure 6.11 and 6.12 for Case A and B, respectively.

From Figure 6.11 it is possible to observe the following:

- During the initial deployment $(\mathrm{t}=0$ to $\mathrm{t}=3 \mathrm{sec})$, the internal pressure shows the presence of a vacuum effect produced by the fall of membrane material due to the action of gravity. The activation of the inflator (at $\mathrm{t}=2 \mathrm{sec}$ ) produced a recovery of the pressure. After few seconds of the activation of the inflator, the internal pressure (gauge pressure) reached a positive value but with a magnitude close to zero. As the inflation progressed, the internal pressure remained approximately constant and close to zero, whereas the internal volume of the inflatable increased linearly.

- At $\mathrm{t}=179 \mathrm{sec}$, right before the failure of the passive restrainers, the internal pressure increased until it reached a local peak that corresponds to the failure of the passive restrainers. Right after the failure of the passive restrainers, the membrane contained in the pre-fold was released, causing an increase of volume, which produced a drop of the internal pressure. However, the inflator system continued providing air mass for filling the internal volume, which produced a recovery of the internal pressure until it reached the target value close to the target value of $6.89 \cdot 10^{3} \mathrm{~Pa}$. Also, during this stage, the volume tended to a constant value close to the target value.

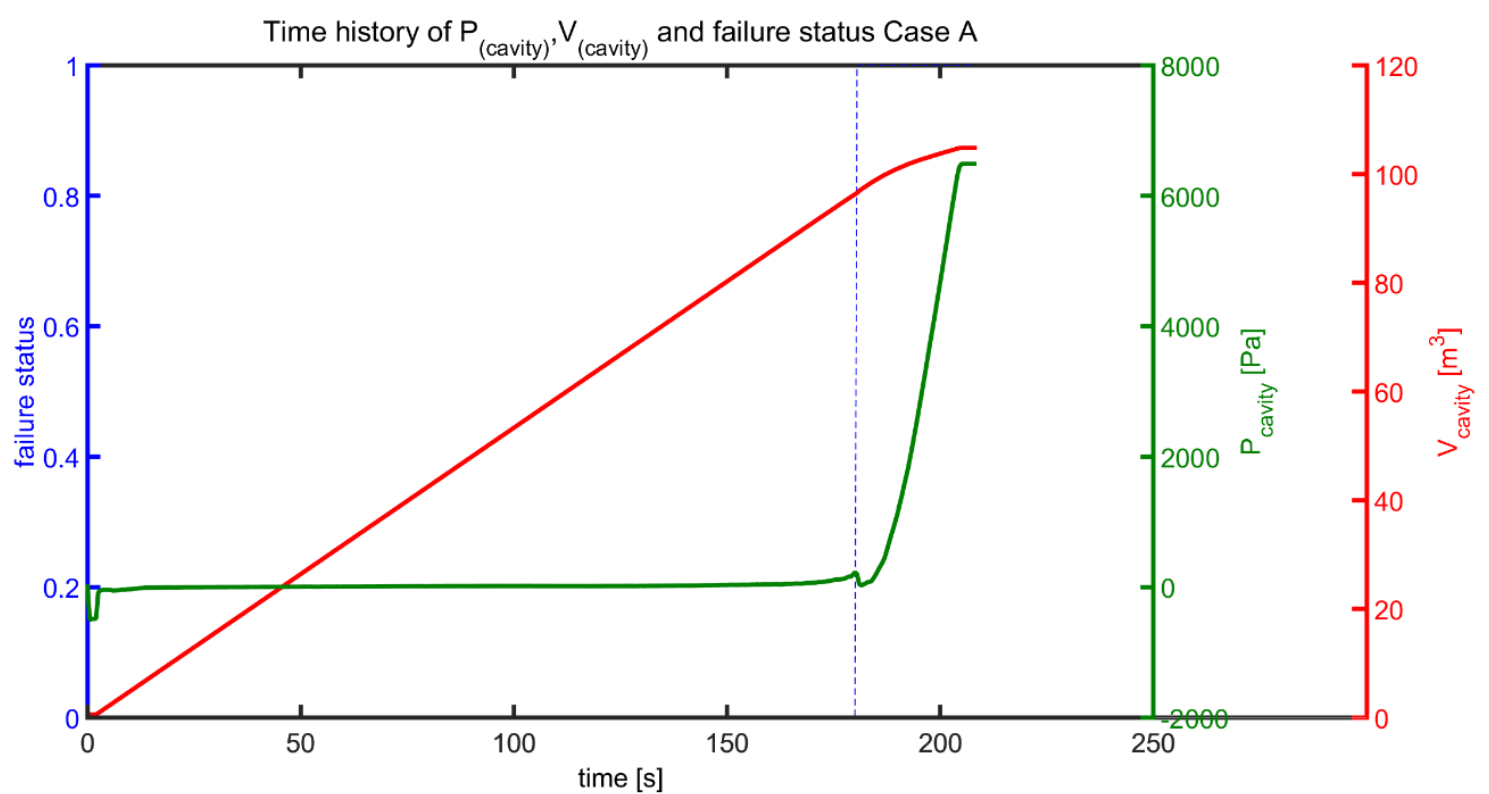

Figure 6.11. Time history of gauge pressure, internal volume and failure status for case $\mathrm{A}$. 
From Figure 6.12, corresponding to Case B, it is possible to see that the internal pressure and the internal volume of the inflatable followed a behavior similar to Case A until the end of the first failure of the passive restrainers placed close to the upper right corner of the tunnel. Since Case B included two pre-folds, from the pressure history, it is possible to see two main local peaks followed by two drops of internal pressure right before and right after the breakage of the passive restrainers. The presence of the third local peak between the two main local peaks indicates that the breakage of the restrainers was not simultaneous. However, the inflator, as in Case A, continued providing air mass and filling the internal volume which produced an increase in the internal pressure. During the pressurization stage, as in Case A, the internal pressure increased until it reached a value close to the target value while the volume tended to a constant value.

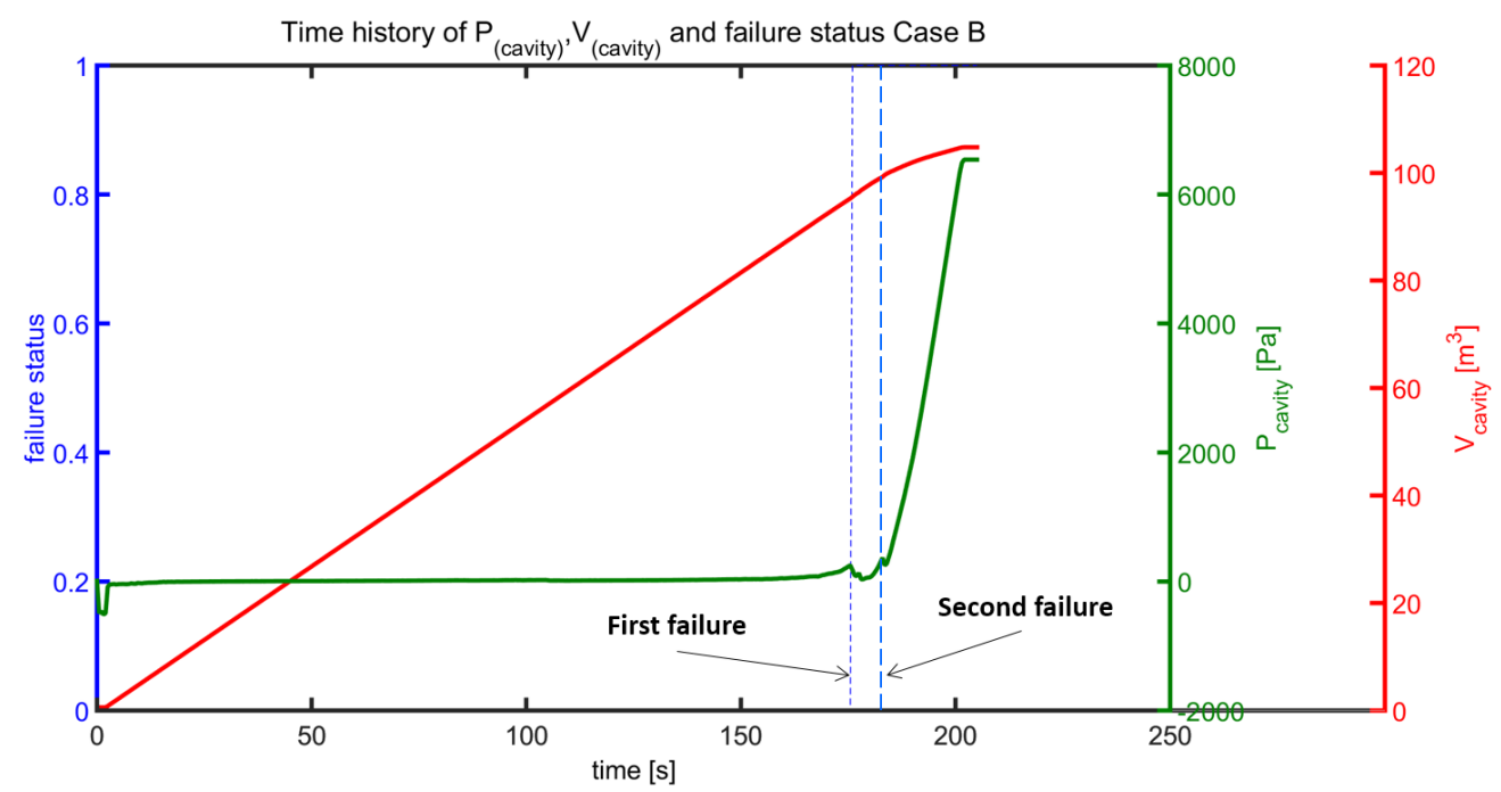

Figure 6.12. Time history of gauge pressure, internal volume and failure status for case $B$.

Figure 6.13 and Figure 6.14 show the time history of the axial forces carried out by each passive restrainer used in the pre-folds corresponding to Cases A and B, respectively. The time history was analyzed to understand the behavior of the passive restrainers during the deployment and inflation process until they broke and released the membrane stored during the controlled deflation process.

For Case A, the behavior of the forces illustrated in Figure 6.13 can be separated into the following parts:

- From $\mathrm{t}=0$ to $\mathrm{t}=25$ seconds, it is possible to see a series of peaks due to fall of the membrane seen during the initial deployment. The magnitude of the forces did not exceed $30 \%$ of the axial strength assigned to the connector elements $(267 \mathrm{~N})$. 
- After the initial deployment is completed, the connectors were unloaded. From $t=25$ to $t=150$ seconds, the inflatable expanded and the behavior of the axial force carried out by the connectors was characterized by small oscillations around a constant load of about $10 \%$ of the axial strength assigned to the connector elements.

- From $t=150$ to $t=180$ seconds, the inflatable started to reach its full shape inside the tunnel, and the connector elements started to get stretched producing an increase of the axial force until they reached their maximum capacity around the 180th second. At that time, the strength of the connectors was reached causing their break and allowed a further release of membrane stored in the first pre-fold, as seen in Figure 6.9.

- After the breakage, from $t=180$ to $t=205$ seconds, the axial forces in the connectors dropped to zero since they broke and cannot take any axial force.

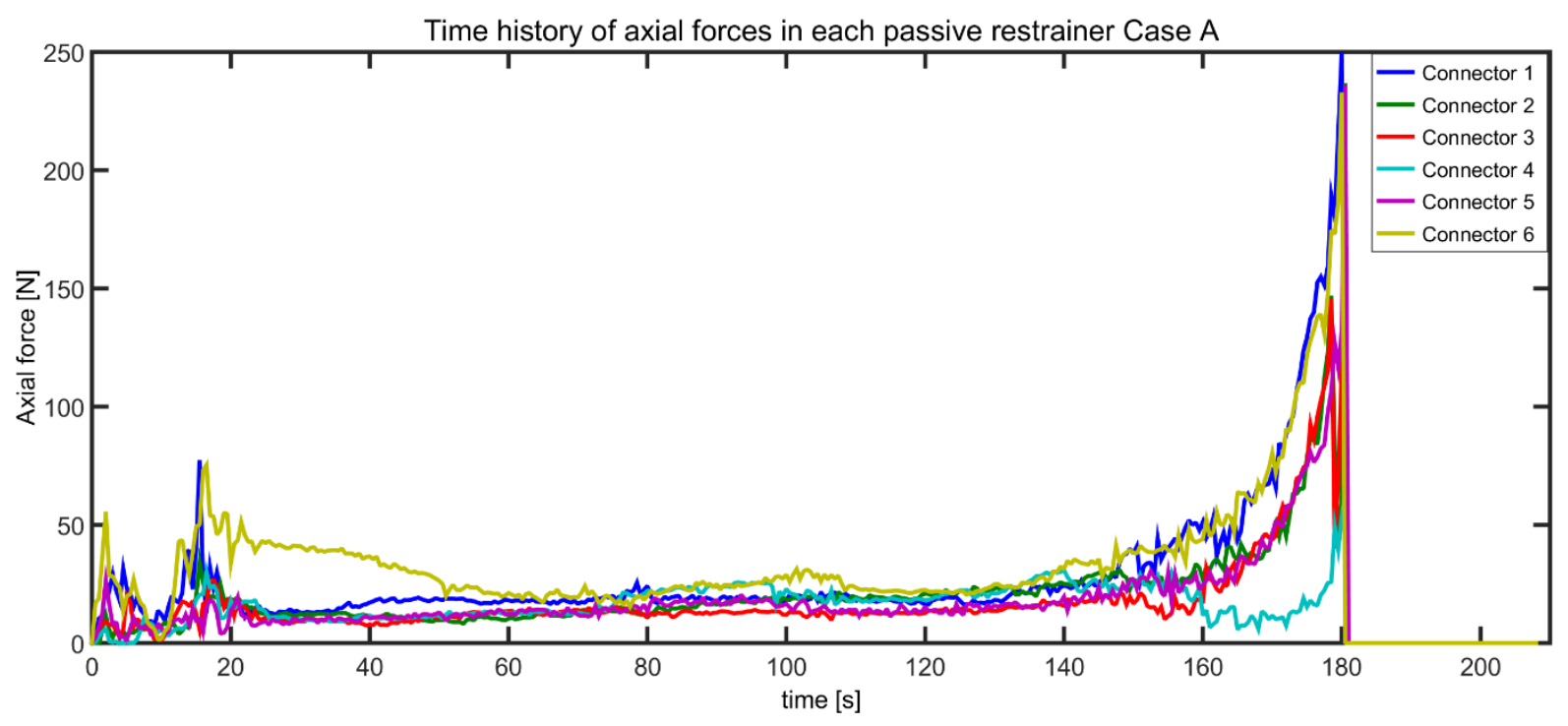

Figure 6.13. Time history of axial force in each connector for case A.

For Case B, the behavior of the forces illustrated in Figure 6.14 can be separated into the following parts:

- From $\mathrm{t}=0$ to $\mathrm{t}=25$ seconds, as in Case $\mathrm{A}$, it is possible to see a series of local peaks originated by the fall of the membrane corresponding to the initial deployment. In this case, the magnitude of the forces did not exceed $26 \%$ of the axial strength assigned to the connector elements $(267 \mathrm{~N})$.

- Similarly to Case A, after the initial deployment was completed, the connectors were unloaded. From $\mathrm{t}=25$ to $\mathrm{t}=150$ seconds, the inflatable expanded and the behavior of the axial force carried out by the connectors is characterized by oscillations around a constant axial load in the range of $1 \%$ to $18 \%$ of the axial strength assigned to the connector elements. 
- From $\mathrm{t}=150$ to about $\mathrm{t}=176$ seconds, the inflatable started to reach its full shape inside the tunnel, and the connector elements related to the first pre-fold started to get stretched producing an increase of the axial force until they reached their maximum capacity around the 176th second. At that time, as in Case A, the strength of the connectors was reached causing their break and producing the release of the membrane stored in the first pre-fold as seen in Figure 6.9.

- From $\mathrm{t}=177$ to about $\mathrm{t}=183$ seconds, the inflatable continued its expansion in the tunnel and the connector elements related to the third pre-fold started to get stretched producing an increase of the axial force until they reached their maximum capacity around the 183rd second. At that time, the strength of the connectors was reached causing the breakage and producing the release of the membrane stored in the third pre-fold, as seen in Figure 6.9.

- Then, after the breakage, from $t=183$ to $t=205$ seconds, the axial forces in the connectors dropped to zero since they broke and cannot take any force.

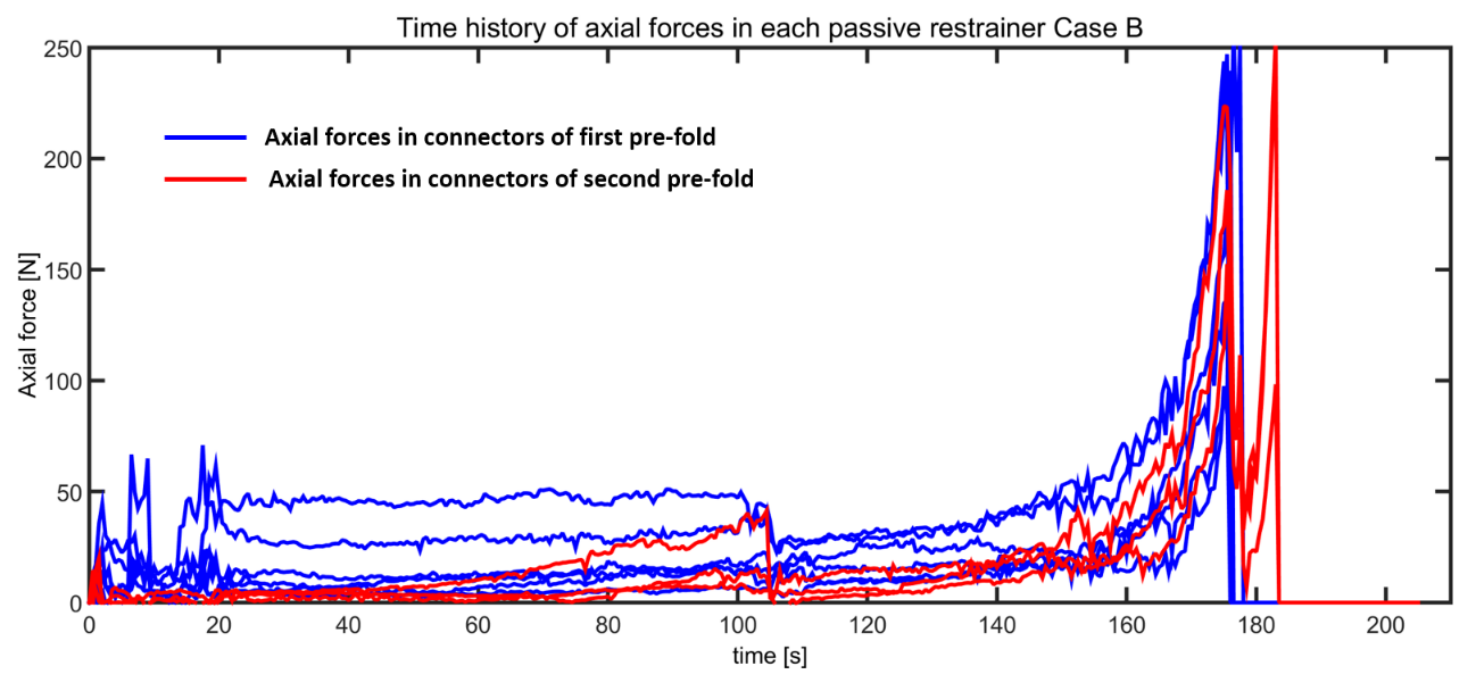

Figure 6.14. Time history of axial force in each connector for case B.

Looking at the overall behavior of the forces carried by the connectors in Cases A and B, it is important to highlight that the timing of the breakage and the maximum force at breakage of the passive restrainers is well within the range of the estimated values originated by Eq. (49) and by the procedure described in Section 6.3. In order to quantify the global conformity and to highlight the improvements reached with the implementation of the controlled release of the membrane, the contact area achieved at the end of the simulations in Cases 0, A and B are plotted in Figure 6.15. In this figure, the dashed lines represent the nominal contact area of the cylindrical portion of the inflatable in the tunnel. 


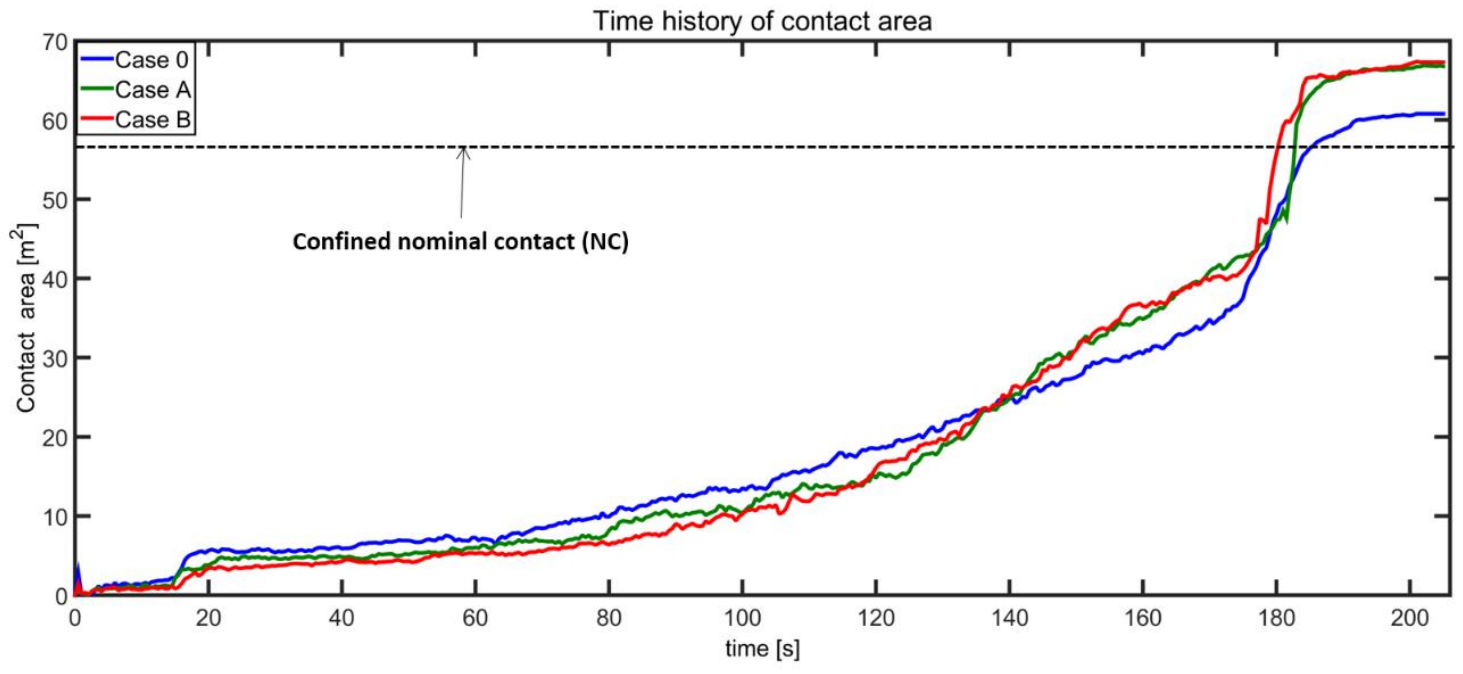

Figure 6.15. Time history of contact area for the three cases (Case 0, Case A, Case B).

From Figure 6.15 it is possible to see that at the end of the simulation, the magnitude of the contact area for Cases 0, A and B exceeded the nominal contact area of the cylindrical portion of the inflatable in the tunnel. The nominal contact (NC) area was evaluated considering the cylindrical region having the same length and radius of the tunnel. Table 6.1 summarizes the percentages of improvement achieved in the three cases. The improvement in the contact area can be attributed to two factors: 1) the confining effect produced by the tunnel in which part of the spherical end caps become part of the cylindrical portion of the inflatable, and 2) the controlled release of the membrane. The percentage of improvement due to confining effect is calculated taking into account the nominal contact area of the cylindrical portion of the inflatable in the tunnel and the contact area of Case 0 , which did not include any pre-folds or passive restrainers. The increase in the contact area produced by the confining effect is due to the fact that part of the spherical end caps became part of the cylindrical portion and thus, increased the final contact area. The percentages of improvement due to the controlled release of the membrane are calculated taking into account the contact area of Case 0, with Cases A and B. The increase in the contact area seen in these two cases is due to the better local conformity in the corners of the tunnel profile. 
Table 6.1. Percentage of improvement of the contact area.

\begin{tabular}{|c|c|c|c|}
\hline Case & Contact Area $\left[\mathrm{m}^{2}\right]$ & Increased Area \% & Improvement due to \\
\hline $\mathrm{NC}$ & 57.72 & - & - \\
\hline 0 & 60.78 & 5 & Confining effect \\
\hline $\mathrm{A}$ & 66.77 & 15 & $\begin{array}{c}\text { Confining effect }+ \\
\text { release of membrane } \\
\text { Confining effect }+ \\
\text { B }\end{array}$ \\
\hline
\end{tabular}

One aspect seen in the simulations of the different cases is the presence of a vacuum effect at the beginning of the simulation during the initial deployment of the inflatable. This effect is plotted in Figure 6.16 which illustrates the internal pressure history during the first 4 seconds of the deployment for Cases $0, \mathrm{~A}$ and $\mathrm{B}$. From this plot it is possible to see that the vacuum pressure increased as the membrane control improved. The peak values of the vacuum pressure obtained from the simulations corresponding to the three cases under investigation are summarized in Table 6.2. This phenomenon can be explained considering the membrane behavior of the inflatable structure during the first few seconds of the unfolding process. In this short period, the sudden fall of the mass of the inflatable structure due to the action of gravity produces inside of the inflatable a slipstreaming effect that causes a zone of low pressure. Since the mass of the inflatable is the same for all three cases, the factor that is attributed to produce different values of the vacuum pressure is the initial internal volume of the inflatable after the completion of the folding. This value is controlled by the compactness of the folded shape. Table 6.2 shows that the initial internal volume of the folded shape decreased as the membrane control improved.

Table 6.2. Values of vacuum pressure and internal volume during initial deployment.

\begin{tabular}{|c|c|c|}
\hline Case & $\begin{array}{c}\text { Vacuum Pressure } \\
(\text { Peak })[\mathrm{Pa}]\end{array}$ & $\begin{array}{c}\text { Initial Internal } \\
\text { Volume }\left[\mathrm{m}^{3}\right]\end{array}$ \\
\hline 0 & 479.38 & 0.65 \\
\hline $\mathrm{A}$ & 492.72 & 0.59 \\
\hline $\mathrm{B}$ & 510.08 & 0.58 \\
\hline
\end{tabular}




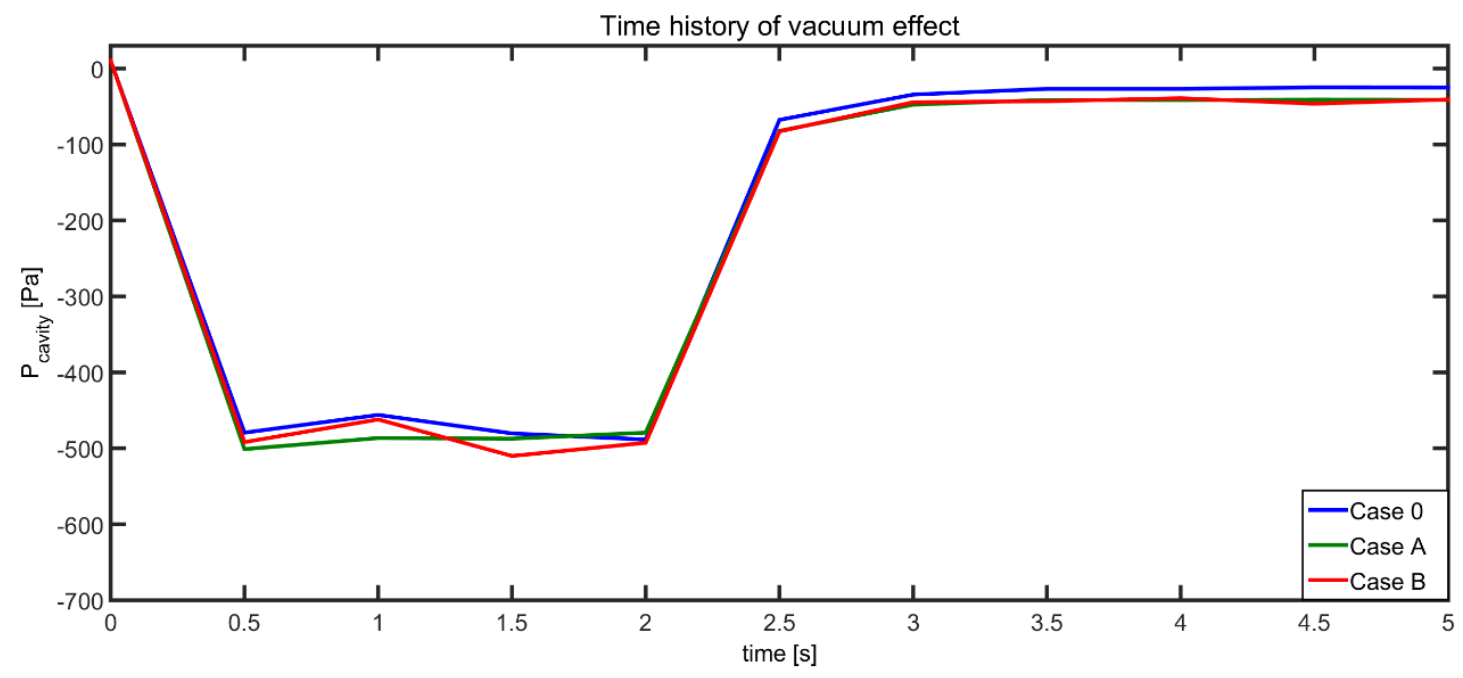

Figure 6.16. Comparison of vacuum effect for the three cases (Case 0, Case A, and Case B).

\subsection{Summary}

Two cases of controlled deflation were presented in this chapter. These cases were used to improve the membrane behavior of the inflatable structure during the initial deployment and inflation. This improvement was achieved by adopting the controlled deflation technique described in Chapter 4 with the inclusion of pre-folds. In the first case (Case A) a single pre-fold was implemented, whereas in the second case (Case B) two pre-folds were implemented. At the end of the controlled deflation with the addition of pre-folding steps, the external lines of each pre-folded segments were held using passive restrainers in order to avoid sliding of the membrane material during the folding process.

The passive restrainers were modeled with connector elements, and their mechanical properties were evaluated. A new folding process was implemented as well. The folding process was implemented by using only two folding planes. The position of the folding planes was selected to perform a symmetric folding sequence. A remarkable improvement was seen not only in terms of final folded shape (the width decreased from $0.32 \mathrm{~m}$ to $0.20 \mathrm{~m}$ and, at the same time, a more uniform longitudinal distribution of the membrane), but also in the reduction of simulation steps (from 19 to 8) and also in terms of the implementation of a feasible way to reach a flatter shape by the application of a vacuum pressure of $700 \mathrm{~Pa}$.. The final folded shape was then positioned on the ceiling of the tunnel using the same technique described in Chapter 5.

The results of two simulations of deployment and inflation implementing the controlled release of the membrane material were described in this chapter as well. The results of the simulations showed how the control of the membrane contributed to reach a higher level of local conformity of the inflatable to the tunnel perimeter. The internal pressure and internal volume time histories were analyzed to understand the 
impact of the inclusion of passive restrainers in the behavior of the inflatable during the inflation and pressurization highlighting the differences between the two cases under investigation (Case A and Case B). Additionally, the time history of the axial forces carried out by the connector elements was plotted to understand how the passive restrainers worked, what was the maximum value of the force that they can achieve before the breakage, and the time at which the breakage took place. The contact area at the end of the simulation was analyzed as well. The simulation results show an increase in the contact area from Case 0 to Cases $\mathrm{A}$ and $\mathrm{B}$. The improvement in the contact area was attributed to the confining effect produced by the tunnel and, to the better local conformity achieved in the corners of the tunnel profile by the gradual release of the membrane. 


\section{Chapter 7. Conclusions and Recommendations for Future Work}

This chapter presents the main conclusions obtained from the simulation results described in the previous chapters. Recommendations for future work are presented as well.

\subsection{Conclusions}

Considering the initial preparation of the finite element model of the inflatable structure studied in this work, the mesh convergence results described in Chapter 3 indicated that although a relatively coarse mesh can predict the stresses with $0.2 \%$ margin of error with respect to the analytical solution, a more refined mesh would be better for implementation of the folding procedures proposed in this work. Therefore, a more refined mesh of $0.05 \mathrm{~m}$ was adopted in this work to reduce the occurrence of inter-element penetration and intersections and also to reduce the final volume of the folded shape. The immediate consequence of using a more refined mesh was the increase of the computational time.

Since one of the main objectives of this work is to obtain a folded shape of the inflatable with the minimum storage volume, two techniques of deflation were implemented: the uncontrolled deflation and the controlled deflation. Although the controlled deflation required more simulation steps and iterations than the uncontrolled deflation, its implementation produced a significant improvement in the resultant deflated shape. The control deflation produced a reduction of the width of the inflatable structure from $0.54 \mathrm{~m}$ to $0.38 \mathrm{~m}$. The controlled deflation contributed to the reduction in the amplitude of wrinkles and also to improve the distribution of the membrane over the surface of the resultant deflated shape.

The simulation of unconfined inflation was used to assess the performance of the factors initially adopted for the definition of the inflator and to evaluate the impact of changes in parameters such as the mass scale factor (MSF) and the mass proportional damping factor $(\alpha)$, and the ambient temperature, as described in Chapter 4. The parametric studies were conducted in order to find a combination of parameters that were able to decrease the computational time of the simulations and, at the same time, to reproduce the behavior of the inflatable structure making it comparable to the experimental observations reported in [7].

The parametric study carried out to evaluate the influence of a mass scale factor with values in the range of 10 to 1000 , indicated that a mass scale factor of 100 was suitable for the simulation of the behavior of the given material of the inflatable. This value was adopted for the different stages of the simulation of the inflatable structure under investigation. This value was high enough to reduce the computational time but at the same time not too high to change the dynamic behavior of the inflatable, particularly at the end of the 
inflation and pressurization stages. Simultaneously, the results also showed that the different values considered for MSF did not have any impact on the stresses on the membrane.

The results of the parametric study of a mass proportional damping factor $(\alpha)$ defined as part of the material properties of the fabric material of the inflatable, indicated that for a mass scale factor of 100, values of $\alpha$ in the range between 0.2 and 0.4 reproduced a more realistic behavior of the membrane. Simulations results with values of $\alpha$ below 0.2 showed significant oscillations and apparent vibrations of the membrane and bouncing at the end of the unconfined inflation and pressurization, while simulations with values of $\alpha$ above 0.4 and up to 1.0 exaggerated the damping effect and therefore changed the overall behavior of the inflatable during the inflation and pressurization.

A parametric study was conducted to understand the influence of the ambient temperature $T_{a}$ on the behavior of the inflator system. The results of this parametric study indicated that the gauge (or internal) pressure of the inflatable and the internal volume increased as the ambient temperature increased. An increase of temperature from $T_{a}=15^{\circ} \mathrm{C}$ to $T_{a}=40^{\circ} \mathrm{C}$ produced an increase of $72 \%$ in the gauge pressure and an increase of $4.9 \%$ in the internal volume of the inflatable. This results suggest that the air mass flow rate of inflator will have to be adjusted to account differences of ambient temperature in order to avoid early or excessive pressurization that could produce overstressing of the fabric material which ultimately can lead to the failure of the inflatable.

The simulation of deployment and confined inflation of the inflatable was presented and discussed in Chapter 5. The results of the FE models were analyzed and compared to the experimental test reported in [7]. An initial comparison of the membrane behavior during the initial unfolding showed a noticeable difference between the simulation results and experimental results. In this initial comparison, the behavior of the membrane in the simulations seemed to be more rigid than the one used during the experiments. This dissimilarity was attributed to the artificial compressive strength adopted in the definition of the constitutive model of the fabric material. In the initial models of confined inflation, this value was assumed to be $0.5 \%$ of the maximum tensile strength. Although this relatively small value contributed to the numerical stabilization of the simulation, by preventing the excessive distortion of unstressed membrane elements and, therefore significantly decreasing the time increment, it also produced an artificially stiffer membrane.

An additional parametric study was conducted changing the value of the artificial compressive strength with the purpose of reaching a membrane behavior in the simulation that was a closer representation of what was observed in the experiments. Results indicated that a decreasing value of compressive strength improved the flexibility of the membrane seen during the initial unfolding and inflation. Further comparison of simulation results with experimental results showed that a value of artificial compressive strength in the 
range of $0.01 \%$ to $0.02 \%$ of the maximum tensile strength resembled more closely the membrane behavior observed in the experiments.

The simulation of deployment and confined inflation was also used to assess the global and local conformity of the membrane to the tunnel profile. Simulation results showed the presence of gaps in the corners of the tunnel due to the lack of uniform distribution of membrane material. This lack of uniform distribution of membrane material was attributed to the uncontrolled release of the membrane material during the initial deployment and inflation. A methodology to improve the lack of uniform distribution of membrane material was introduced and discussed in Chapter 6. This methodology included the adoption of the controlled deflation technique described in Chapter 4 with the addition of pre-folds held by passive restrainers. The implementation of a vacuum pressure contributed to achieve an even flatter deflated shape. A remarkable improvement was also reached in terms of the final folded shape and the reduction of simulation steps. The inclusion of these passive restrainers was intended not only to preserve the position of the pre-folds during the folding procedure but also to produce a gradual release of the membrane during the latter stages of the inflation process. The simulation results showed how the control of the membrane during the deflation, folding, deployment, and inflation contributed to reach higher levels of local conformity by closing gaps around the corners of the tunnel perimeter, which translated in an increase of contact area. The implementation of only one pre-fold produced an increase of the $15 \%$ of the resultant nominal contact area whereas the implementation of two pre-folds produced an increase of the $16 \%$ of the same area. Results also showed that the simplified geometry of the inflatable adopted for the simulations presented in this work was able to reach similar levels of global and local conformity as the levels reached with a fitted shape of the inflatable adopted in the experiment reported in [7].

\subsection{Recommendations for Future Work}

Although several parametric studies were conducted in this work, the following additional studies are suggested to improve the understanding and predictability of finite element models of inflatable structures subject to confined inflation, including:

- A parametric study to evaluate the influence of the stiffness proportional Rayleigh damping coefficient $\beta$.

- A parametric study to evaluate the influence of the magnitude of the contact penalty factor.

- A parametric study to evaluate the influence of the bulk viscosity coefficient.

- The evaluation of alternative folding sequences to understand the impact that other folding sequences could have on the final inflated shape of the inflatable in confined conditions in terms of global and local conformity. 
- The innovative techniques for controlling the membrane of the inflatable developed in this work demonstrated that it is possible to achieve higher levels of local conformity and they could be applied to other tunnel profiles with more intricate shapes. 


\section{Appendix A. Deployment and Inflation of a Segment of Inflatable Boom}

\subsection{Introduction}

This Appendix presents additional exploratory work conducted to simulate the deployment and inflation of a segment of an inflatable boom. The objective of this supplemental work was to explore the simulation of other folding procedures for producing a two-zig-zag folding and rolling. These two folding patterns are simulated implementing alternative techniques to reproduce the final folded shape used in a small-scale exploratory experiment. The resultant folded shapes are then used to simulate the vertical deployment and inflation. The simulation results are then compared to the results obtained in the experiments.

\subsection{Model Definition and Properties}

A model for a segment of an inflatable boom is developed. The deflated geometry of the inflatable boom is defined by two surfaces called upper and lower surfaces. The surfaces connect in a middle plane via linear transitions running along the perimeter as shown in Figure 8.1 (a). The total length of the inflatable is equal to $1.016 \mathrm{~m}$ and the width is equal to $0.457 \mathrm{~m}$. The initial geometry of the inflatable was created using a three-dimensional deformable shell using Abaqus/CAE. The surface was then partitioned into five subsurfaces as shown in Figure 8.1 (b). The partitions were created in order to identify folding surfaces and folding lines that were used as references during the folding procedure.

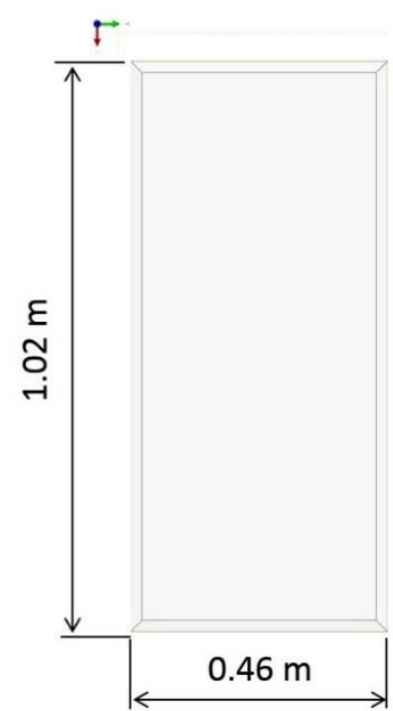

(a)

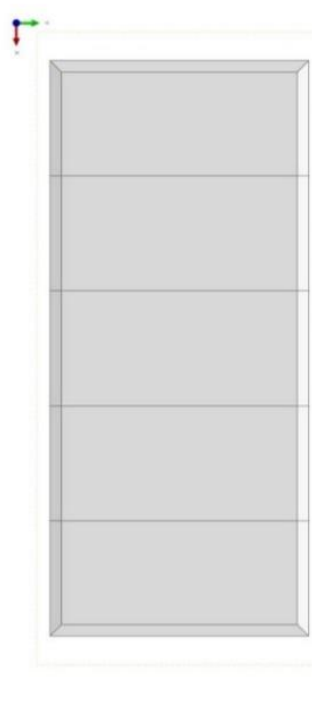

(b)

Figure 8.1. (a) Inflatable boom geometry and dimensions; (b) Partitions. 
The membrane of the inflatable is a single layer of low-density polyethylene (LDPE) with a thickness of $t=0.0000508 \mathrm{~m}$ and density of $\rho=920 \frac{\mathrm{Kg}}{\mathrm{m}^{3}}$. The mechanical properties of LDPE adapted from information available in the literature [39-54]. The LDPE material is assumed to behave as an orthotropic material with tensile strengths in the warp and fill directions. The mechanical behavior under tensile loads is shown in Figure 8.2.

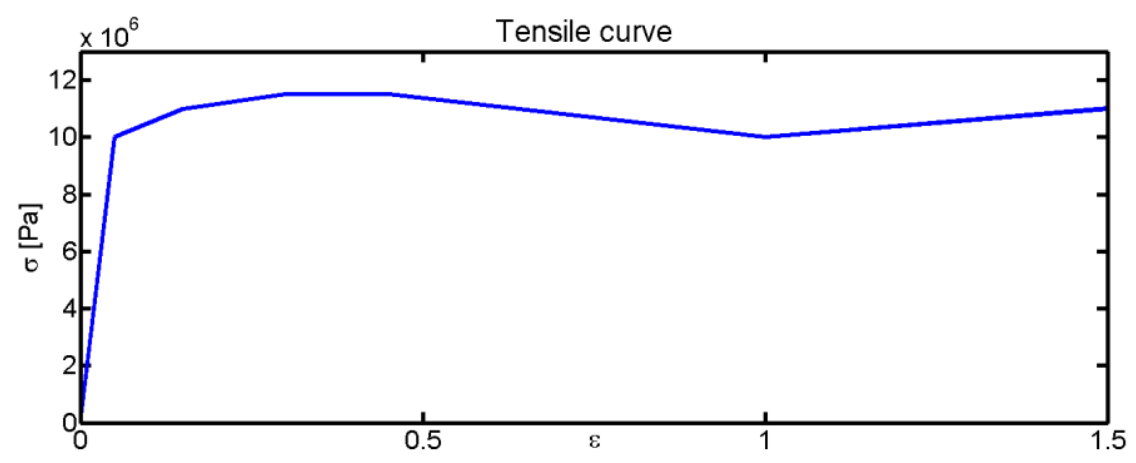

Figure 8.2. Constitutive model of membrane material.

The membrane material is assumed not to have stiffness under compression. However, the stability and the convergence of the FE models require the definition of an artificial compressive strength to prevent excessive distortions or the collapse of membrane elements. In the models of the inflatable, a compressive strength equal to $0.01 \%$ of the maximum tensile strength was assigned to the constitutive model.

During the simulation, the inflatable will interact with a flat surface called "base" which is representative of a solid surface on which the folding procedures will take place. The base is represented by a rectangular shape surface of $1.00 \mathrm{~m}$ by $2.0 \mathrm{~m}$.

\subsection{Generation of FE models}

The membrane of the inflatable is simulated using M3D3 membrane elements. The implementation of folding procedures required a refined mesh to obtain a very compact folded shape and, at the same time, to prevent inter-element penetration and intersections. The nominal shape illustrated in Figure 8.1 is modeled using 21904 elements. The FE model of the base was created with three-dimensional rigid shell surfaces generated in Abaqus/CAE. The base was considered non-deformable, it was meshed using linear quadrilateral rigid elements R3D4, and it is formed by 200 elements with a size of $0.1 \mathrm{~m}$. The meshes of the inflatable and the base are shown in Figure 8.3. 


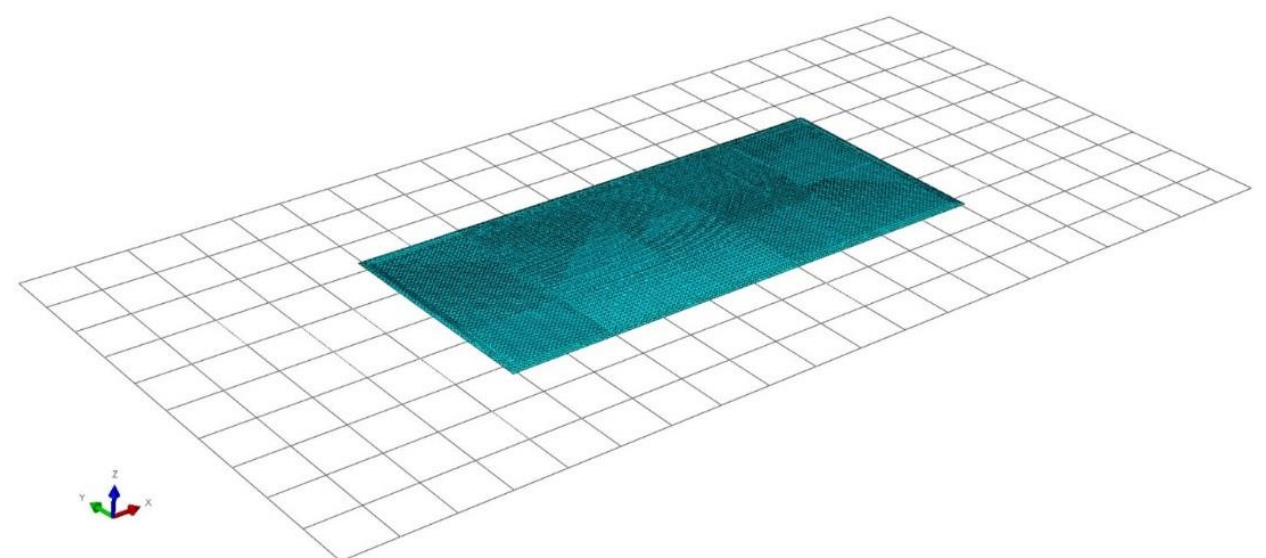

Figure 8.3. Meshes of the inflatable and base.

After the creation of the components of the FE model, all the nodes and elements of meshed geometries were renumbered through HyperMesh. The model also required the definition of a metric file used as reference shape to reach the final shape at the end of the inflation without the wrinkles originated by the folding procedure. The metric file was created taking into account the inflated shape obtained pressurizing the inflatable using an internal pneumatic pressure of $P=2000 \mathrm{~Pa}$ (or $0.29 \mathrm{psi}$ ), which corresponds to the value of the internal (or gauge) pressure measured during the experimental test described in the following sections.

\subsection{Folding Methods}

Two folding patterns were simulated: a two-zig-zag folding and rolling. The partitions that define the subsurfaces and folding lines implemented in these two folding patterns are shown in Figure 8.4. The total surface is divided into five sub-surfaces, and each sub-surface is delimited by two folding lines (FL).

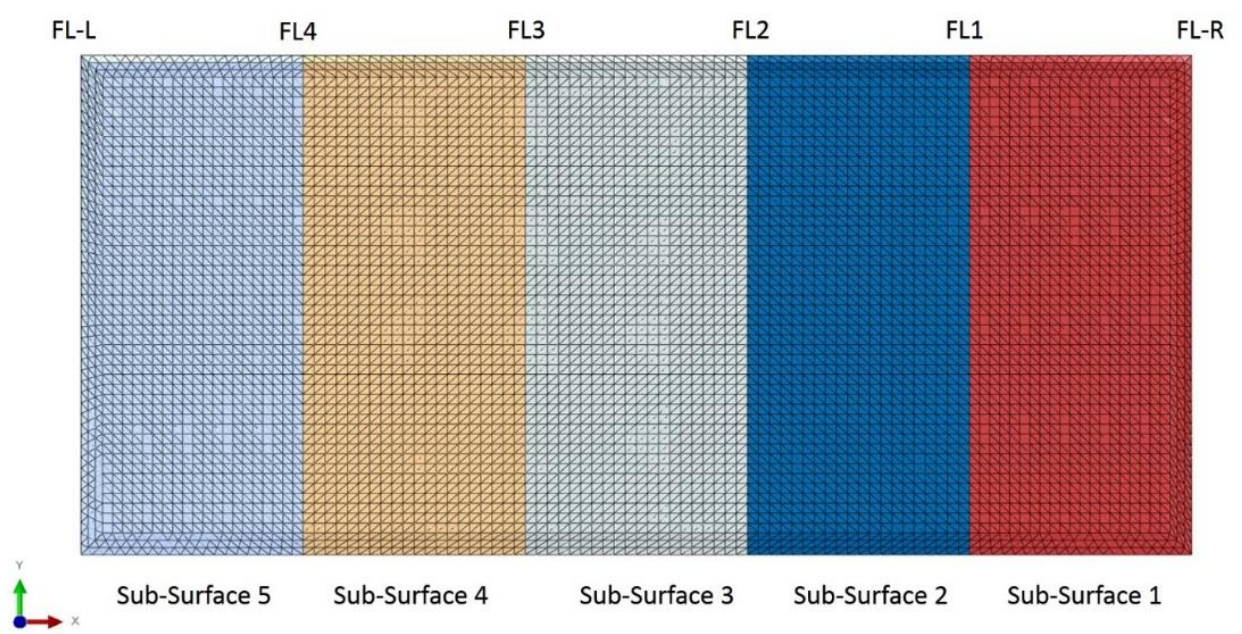

Figure 8.4. Sub-surfaces and folding lines. 


\subsubsection{Zig-zag Folding}

The two-zig-zag folding sequence is illustrated in Figure 8.5 was completed in five different simulations. At the end of each simulation, the coordinates of the resultant shape were exported first to Abaqus/CAE and then to Hypermesh to inspect the mesh and detect if the membrane elements were affected by interelement penetrations and intersections, and in such case, correct them before proceeding for the next simulation. All the simulations of this folding sequence were performed using an MSF equal to 100, a mass proportional damping factor $\alpha=0.4$ and no CPF. Contact interactions and friction were defined between the inflatable boom and the base $(\mu=0.50)$, and between the inflatable and itself $(\mu=0.20)$. The gravity was kept active during all the zig-zag folding simulations. The sequence of steps performed to reach the two-zig-zag folded shape are explained below:

1) The first simulation was performed in only one step as shown in Figure 8.5 (a). In this step, the inflatable structure was initially flattened by the action of gravity applied to all membrane elements along the Zaxis.

2) In the second simulation, shown in Figure 8.5 (b-c), the sub-surfaces from 1 to 4 were defined as rigid bodies and the folding lines (FL-L and FL4) were constrained to move in any direction. In this simulation, the reference node of the rigid surface was set to translate vertically along the Z-axis $\left(U_{3}=\right.$ $0.04 \mathrm{~m})$ and then, the reference node was set to translate horizontally along the $\mathrm{X}$ axis $\left(U_{1}=\right.$ $-0.38 \mathrm{~m})$.

3) In the third simulation illustrated in Figure 8.5 (d), all the surfaces were defined as membrane elements. The action gravity completed the first zig-zag fold.

4) In the fourth simulation illustrated in Figure 8.5 (e-f), the sub-surface 1 was defined as a rigid body, and the folding lines (FL-L, FL3, and FL4) were constrained to move in any direction. During the simulation, the reference node of the rigid surface was set to translate vertically along the Z-axis $\left(U_{3}=\right.$ $0.04 \mathrm{~m}$ ) and subsequently, the reference node of the rigid surface was set to translate horizontally along the $\mathrm{X}$ axis $\left(U_{1}=-0.40 \mathrm{~m}\right)$.

5) In the fifth simulation shown in Figure $8.5(\mathrm{~g})$, all the surfaces were defined again as membrane elements and the action of gravity completed the second zig-zag fold. 


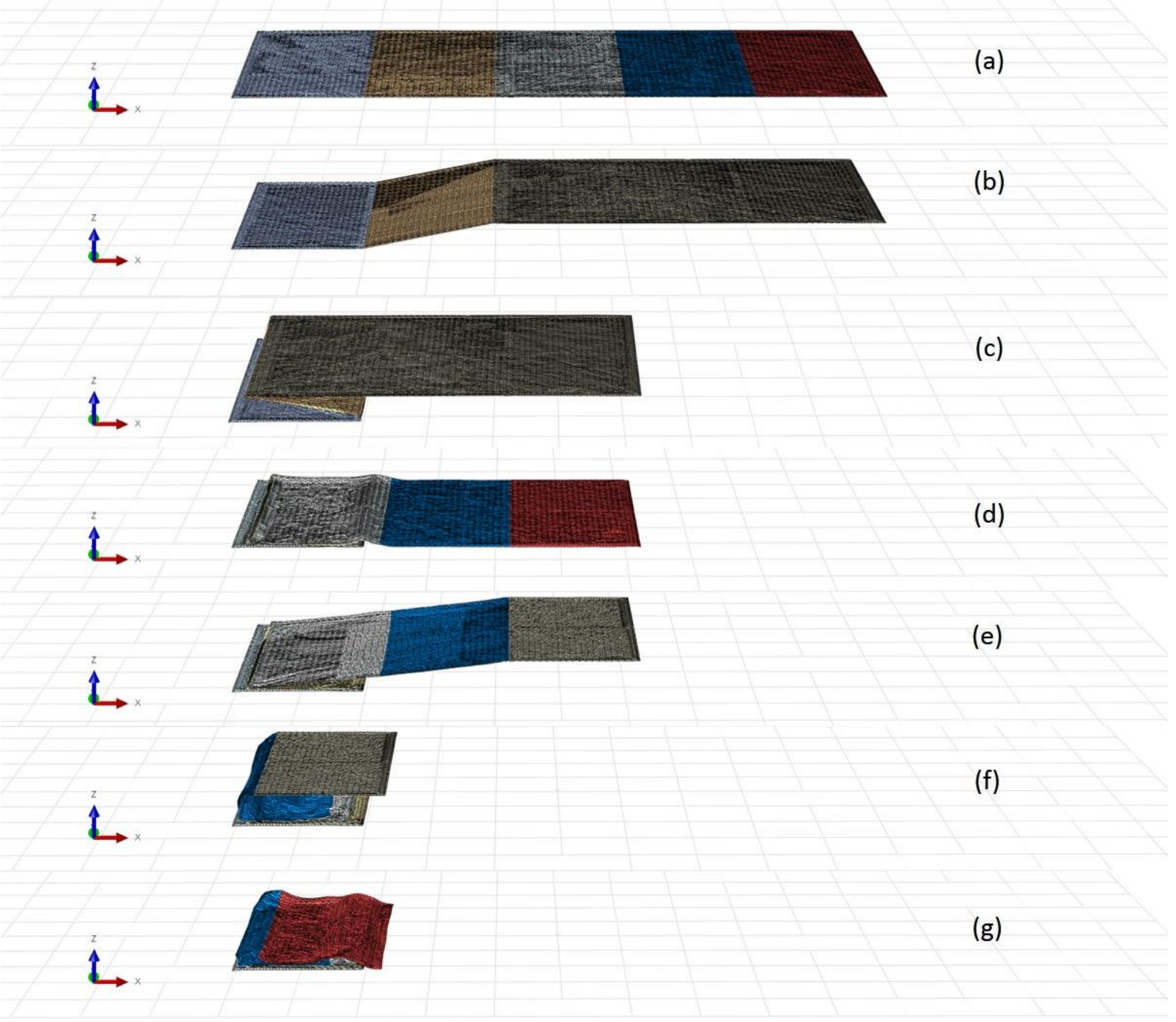

Figure 8.5. Two zig-zag folding sequence, main folding steps.

\subsubsection{Folding by Rolling}

The folding sequence illustrated in Figure 8.6 was completed in ten different simulations. As in the zig-zag folding, at the end of each simulation, the coordinates of the resultant shape were exported first to Abaqus/CAE and then to Hypermesh for inspection and detection of inter-element penetrations and intersections before proceeding for the subsequent simulations. All the simulations of the folding sequence were performed using an $\mathrm{MSF}=100$, a mass proportional damping factor $\alpha=0.4$ and no contact penalty factor. Contact interactions and friction were defined between the inflatable boom and the base $(\mu=0.50)$, and between the inflatable boom and itself $(\mu=0.20)$. The sequence of steps performed to reach the folded shape, are explained below: 
1) The first simulation illustrated in Figure 8.6 (a-d) was performed in four steps. The inflatable structure was initially flattened by the action of gravity applied to all membrane elements along the Z-axis. Then, translational and rotational boundary conditions were applied to the nodes on the folding line FL-L. The nodes were set to translate vertically along the Z-axis $\left(U_{3}=0.02 \mathrm{~m}\right)$, then to rotate around the $\mathrm{Y}$ axis $\left(U_{5}=30^{\circ}\right)$ and, as the last step, to translate horizontally along the $\mathrm{X}$ axis $\left(U_{1}=0.40 \mathrm{~m}\right)$.

2) In the second simulation illustrated in Figure $8.6(\mathrm{e})$, the action of gravity completed the first fold.

3) In the third simulation shown in Figure 8.6 ( $\mathrm{f}-\mathrm{h}$, the folding lines FL-L and FL3 were held using connector elements to keep the position of the first fold during the second rolling procedure. The same boundary conditions applied to the nodes on the line FL-L were applied now to the nodes on the folding line FL4.

4) Only gravity was applied in the fourth simulation as shown in Figure 8.6 (e). At the end of this simulation, the second fold was completed.

5) The fifth simulation illustrated in Figure $8.6(\mathrm{j}-\mathrm{k})$ was performed in three steps. The folding lines FL4 and FL2 were held using connector elements for the same reason explained previously. The action of gravity initially flattened the inflatable and then, translational and rotational boundary conditions were applied to the nodes on the folding line FL1. The nodes were set to translate vertically along the Z-axis $\left(U_{3}=0.02 \mathrm{~m}\right)$, then, to rotate around the $\mathrm{Y}$ axis $\left(U_{5}=-10^{\circ}\right)$ and, as the last step, to translate horizontally along the $\mathrm{X}$ axis $\left(U_{1}=-0.40 \mathrm{~m}\right)$.

6) In the sixth simulation, the third fold was completed by the action of gravity as shown in Figure 8.6 (1).

7) The seventh simulation was performed in three steps as illustrated in Figure 8.6 (m-o). The folding lines FL1, FL2, and FL3 were constrained to move in any direction. Translational boundary conditions were applied to the nodes on the folding line FL-R. The nodes were set to translate vertically along the Z-axis $\left(U_{3}=0.06 \mathrm{~m}\right)$ and then to translate along the $\mathrm{X}$ axis $\left(U_{1}=-0.40 \mathrm{~m}\right)$.

8) In the eighth simulation only gravity was applied to all membrane elements as shown in Figure 8.6 (p)

9) In the ninth simulation, all the inflatable was defined as a rigid body. The master reference node was set to rotate around the $\mathrm{Y}$-axis $\left(U_{5}=180^{\circ}\right)$ as shown in Figure $8.6(\mathrm{q})$.

10) The last simulation was performed in four steps as shown in Figure 8.6 (r-s). Gravity, as always, was applied in the first step. Translational boundary conditions were applied to the nodes on the folding line FL-R. The nodes were set to translate vertically along the Z-axis $\left(U_{3}=0.05 \mathrm{~m}\right)$, and then, to translate along the $\mathrm{X}$ axis $\left(U_{1}=-0.39 \mathrm{~m}\right)$. At the end of the simulation, all constrains applied to the nodes on the folding line FL-R were removed to obtaining the fourth fold. 


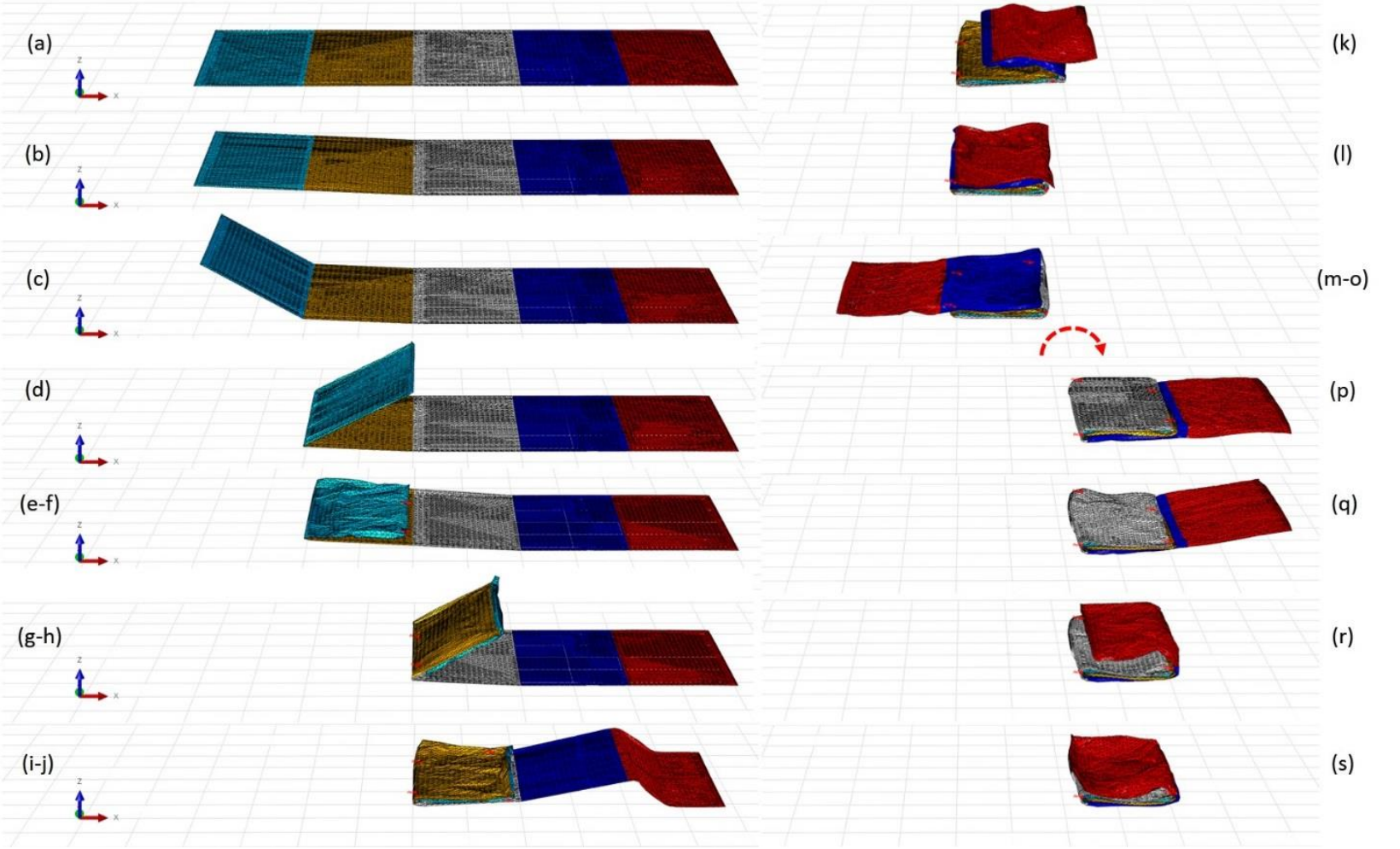

Figure 8.6. Rolling sequence, main folding steps.

\subsection{Simulation of Deployment and Inflation}

The simulation of the deployment and the inflation process of the two folded shapes described above were performed and compared to the results of a small-scale experiment. The sequence of deployment and inflation starts with the folded shape fixed along the top edge of the inflatable. During the deployment, the fixed nodes were not allowed to translate but were allowed to rotate. Gravity was applied from the beginning of the simulation, and the inflator was activated at the end of the deployment. The inflation was performed using an MSF of 20, a mass proportional damping factor $\alpha=0.8$ and no contact penalty factor. The entire initial deployment and inflation sequence was set to take place in 120 seconds plus 5 additional seconds for deactivation of the inflator and pressure stabilization. A mass flow rate of $0.000605 \frac{\mathrm{kg}}{\mathrm{sec}}$ was implemented in the inflator definition. Figure 8.7 and Figure 8.8 show a sequence of images corresponding to the initial deployment of the zig-zag folding and rolling. Figure 8.9 shows a sequence of images of the inflation process. These images include the simulation results compared to the results obtained from the exploratory small-scale experiment. 


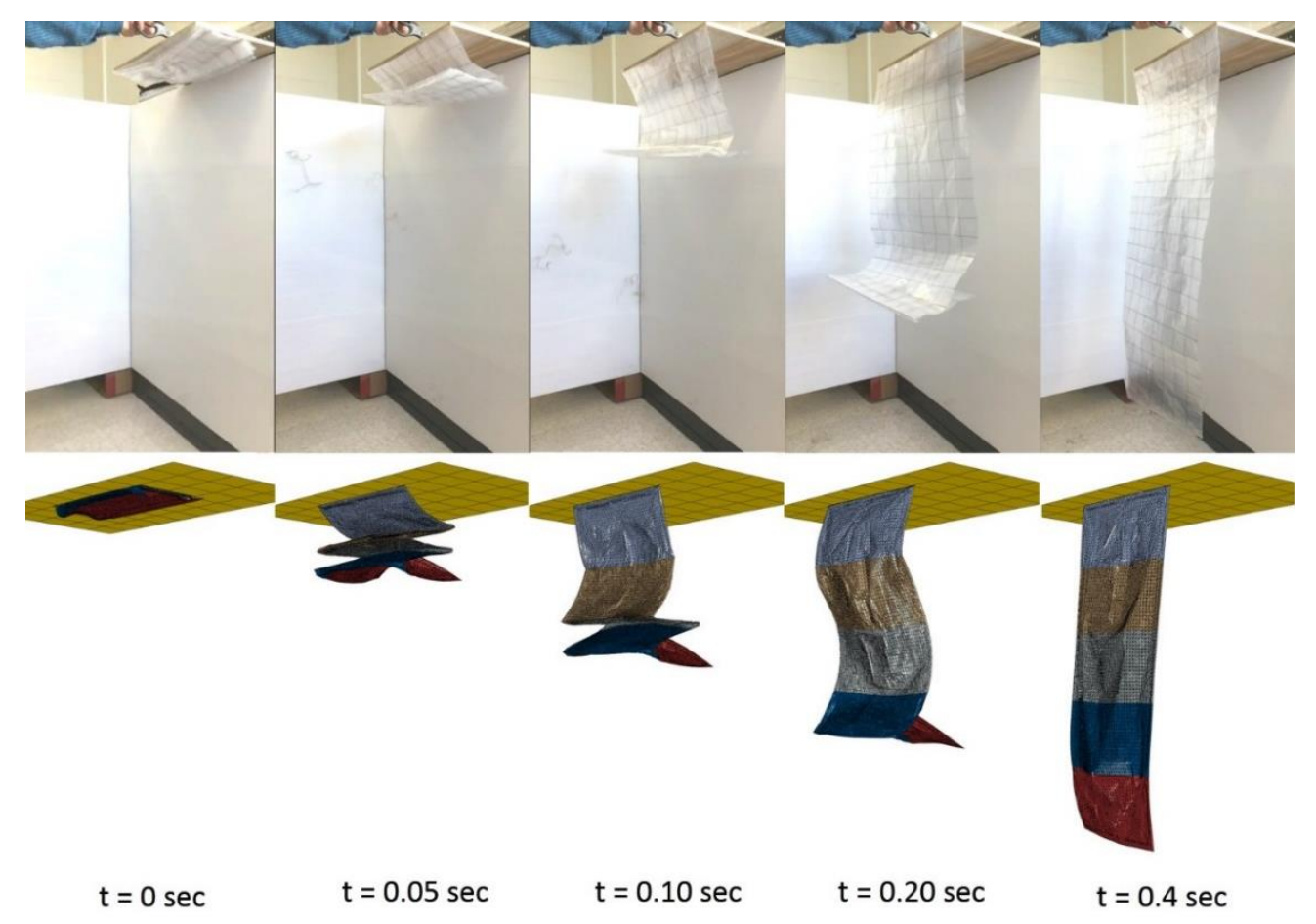

Figure 8.7. Deployment of two-zig-zag folding.

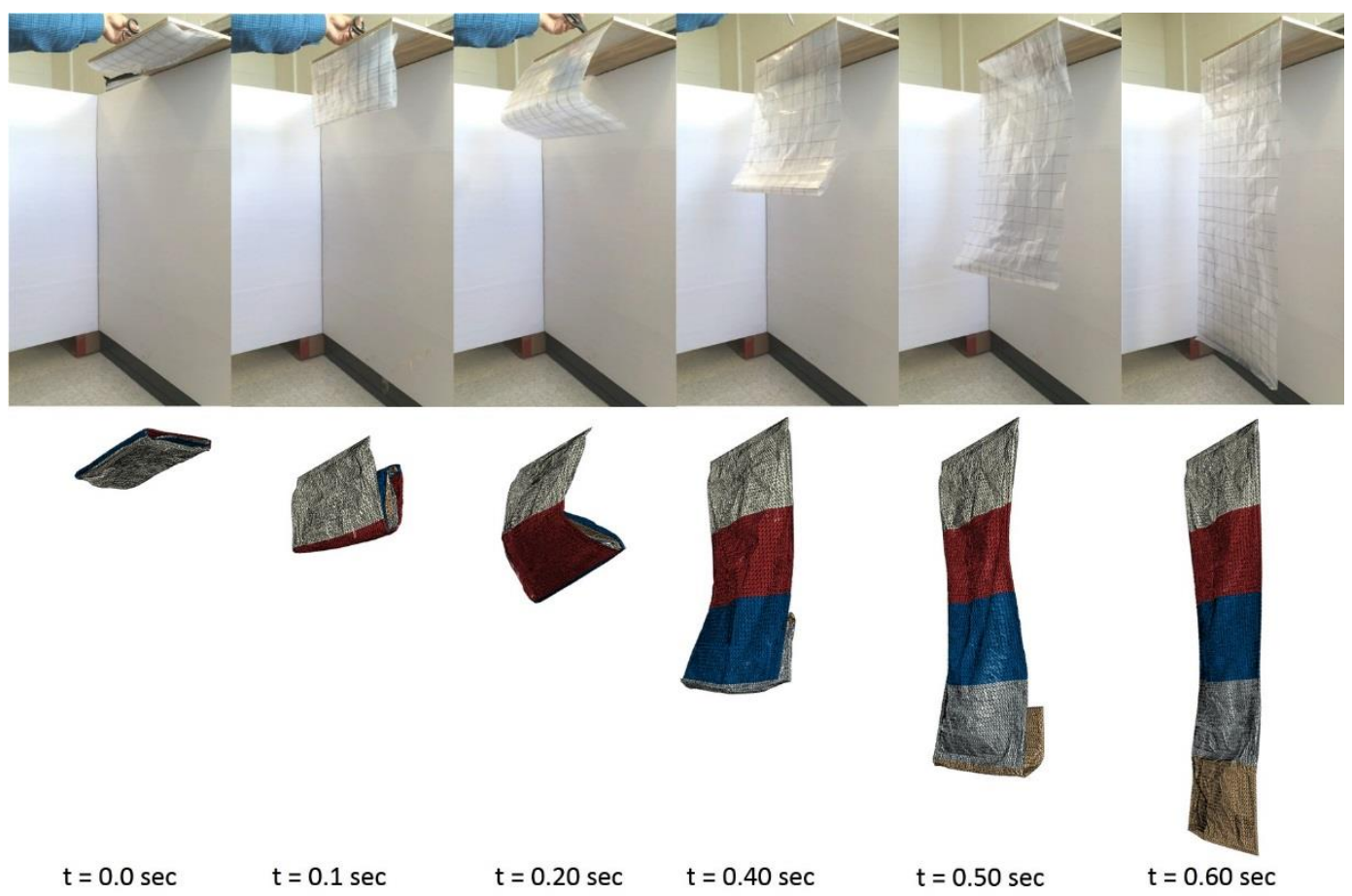

Figure 8.8. Deployment of rolling. 

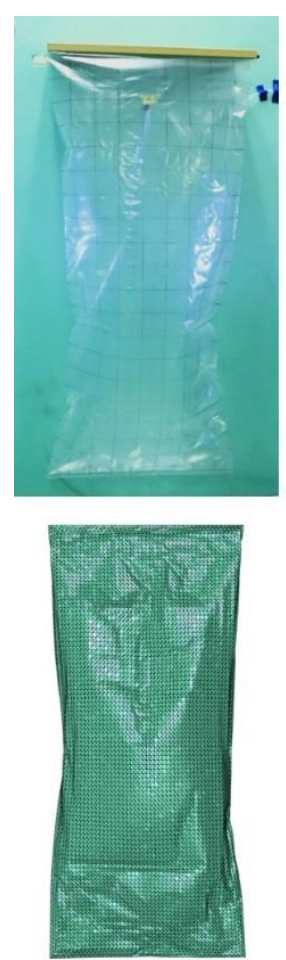

$\mathrm{t}=67 \mathrm{sec}$
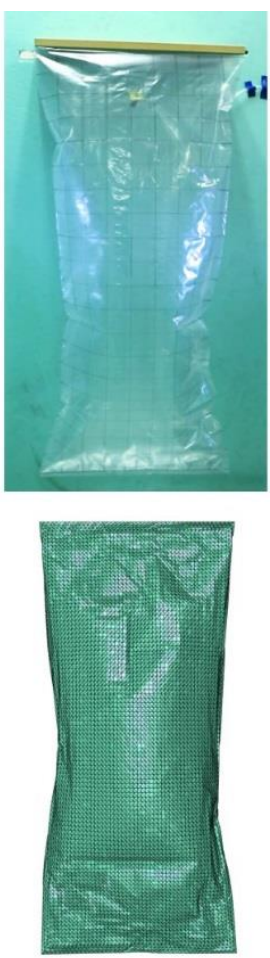

$t=76 \mathrm{sec}$
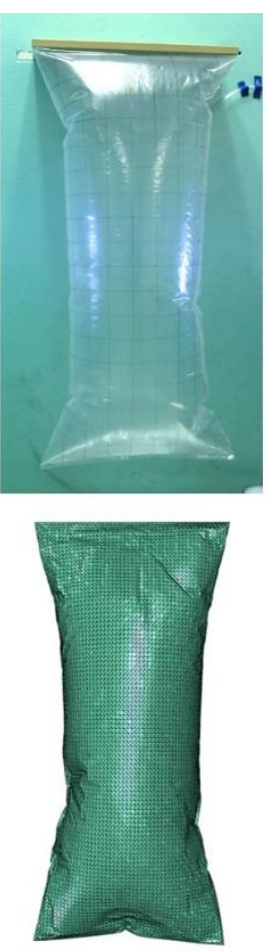

$\mathrm{t}=110 \mathrm{sec}$
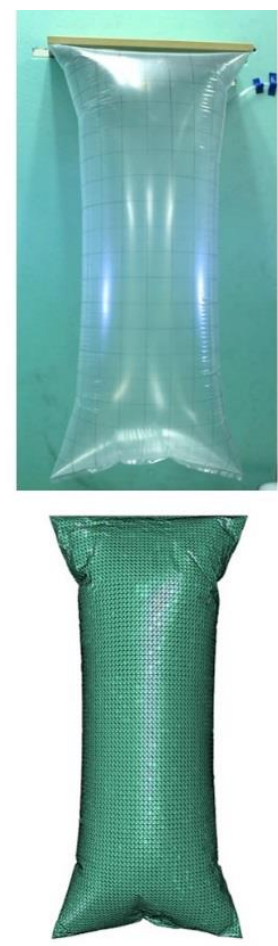

$\mathrm{t}=118 \mathrm{sec}$
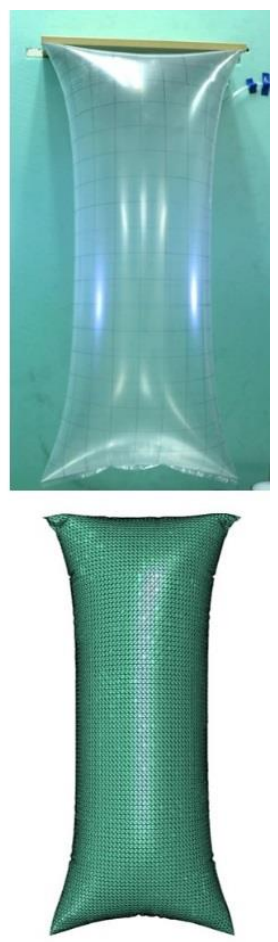

$t=125 \mathrm{sec}$

Figure 8.9. Sequence of inflation.

From Figure 8.7 to 8.8 it is possible to observe that during the deployment of the inflatable, the FE predictions and the experimental results don't match exactly. These results are attributed to the following two factors: a) the artificial compressive strength adopted for the simulations; and b) the shear strength adopted for the membrane material. Moreover, since the material used in the prototype is very light, the surrounding air present during the deployment probably influenced the behavior of the prototype by creating a relatively viscous environment in which the total mass of the inflatable could not totally overcome the resistance produced by the air. This effect is not captured by the FE model since aerodynamic drag was not incorporated. A more accurate constitutive model corresponding to the actual material used in the experiments would be needed to achieve a better match between the simulation and the experimental results during the deployment. On the other side, the simulation of the inflation matched the behavior seen in the experiment relatively well as illustrated in Figure 8.9. The air mass flow rate adopted in the simulation was adequate to reach the same pressure measured in the experiment at the end of the inflation. 


\section{References}

[1] The Blue Ribbon Panel on Bridge and Tunnel Security (2003). Recommendations for Bridge and Tunnel Security. Publication FHWA-IF-03-036. U.S. Department of Transportation.

[2] TCRP Report 86/NCHRP Report 525 (2006). Public Transportation Security. Volume 12. Making Transportation Tunnels Safe and Secure. Transportation Research Board of the National Academies, Washington, D.C.

[3] Rabkin N., (2007). Passenger Rail Security, Federal Strategy and Enhanced Coordination Needed to Prioritize and Guide Security Efforts. Publication GAO-07-583T, U.S. Government Accountability Office.

[4] Inouye, R.R., and Jacobazzi, J.D. (1992).The Great Chicago Flood of 1992. Civil Engineering ASCE 62(11): 52-55.

[5] Fountain, H. (2012). Holding Back Floodwaters with a Balloon. The New York Times/Science 3 Supplement. URL: http://www.nytimes.com/2012/11/20/science/creating-a-balloonlike-4 plug-tohold-backfloodwaters.html.

[6] Zimmerman, R. (2014). Planning restoration of vital infrastructure services following Hurricane Sandy: Lessons Learned for Energy and Transportation. Journal of Extreme Events, 19 1(1) 145004:1-38.

[7] Martinez, X., Davalos, J., Barbero, E.J., Sosa, E.M., Huebsch. W., Means, K., Banta, L., and Thompson, G.J. (2012). Inflatable Plug for Threat Mitigation in transportation Tunnels. Proceedings of the Society for the Advancement of Material and Process Engineering (SAMPE) Conference, Baltimore, MD, May 21-24, 2012.

[8] Barbero, E.J., Sosa, E.M., Martinez, X., and Gutierrez, J.M. (2013). Reliability Design Methodology for Confined High-Pressure Inflatable Structures. Engineering Structures, 51:1-9.

[9] Sosa, E.M., Barbero E.J., and Thompson, G.J. (2014). Design and Testing Inflatable Plugs for Flood Containment in Tunnels. Revista Internacional de Desastres Naturales, Accidentes e Infraestructura Civil, 14(1-2): 39-57.

[10] Barbero, E.J., Sosa, E.M., and Thompson, G.J. (2013). Testing of Full-Scale Confined Inflatable for the Protection of Tunnels. Proceedings of the VI International Conference on Textile Composite and Inflatable Structures, Structural Membranes 2013, Munich, Germany, October 9-11, 2013.

[11] Sosa, E.M., Thompson, G.J., and Barbero E.J. (2014). Testing of full-scale inflatable plug for flood mitigation in tunnels. Transportation Research Record: Journal of the Transportation Research Board, 2407, 2:59-67, 2014. 
[12] Sosa, E.M., Thompson, G.J., Barbero, E.J., Ghosh, S., and Peil, K.L. (2014). Friction Characteristics of Confined Inflatable Structures. Friction, 2(4):365-390.

[13] Sosa, E.M., Thompson, G.J., and Barbero E.J. (2017). Experimental Investigation of Initial Deployment of Inflatable Structures for Sealing of Rail Tunnels. Tunneling and Underground Space Technology, 69: 37-51.

[14] Wong, J.C. (2013). Modeling of High Pressure Confined Inflatable Structures, Ph.D. Dissertation, Morgantown WV.

[15] Sosa, E.M., Wong, J.C., Adumitroaie, A., Barbero, E.J., and Thompson, G.J. (2016). Finite Element Simulation of Deployment of Large-Scale Confined Inflatable Structures. Thin-Walled Structures, 104:152-167.

[16] Wang, J.T., and Nefske, D.J. (1988). New CAL3D Airbag Inflation Model. SAE Technical Paper Series 880654. International Congress and Exposition, Detroit, MI, February 29-March 4, 1988.

[17] Wang, J.T. (1995). An Analytical Model for an Airbag with Hybrid Inflator. General Motors Research \& Development Center, Crashworthiness and Occupant Protection in Transportation System, ASME, AMD-210/BED-30.

[18] Wang, J.T. (2005). Dynamic Deployment Simulations of Inflatable Space Structures. Proceedings of 5th International Conference on Computation of Shell and Spatial Structures, Salzburg, Austria, June $1-4,2005$.

[19] Katsumata, N., Natori, M.C., Yamakawa, H. (2014). Analysis of Dynamic Behavior of Inflatable Booms in Zig-zag and Modified Zig-zag Folding Patterns. Acta Astronautica, 93:45-54.

[20] Roe L.A. (2001). Chapter14: Inflation Systems. Gossamer Spacecraft: Membrane and Inflatable Structures Technology for Space Application. AIAA, Editor: Jenkins, C.H.M, Virginia, USA.

[21] Lee, J.K., Ha, W.P., Lee, J.H., Chae, D.B., and Kim, J.H. (2009). Validation Methodology on Airbag Deployment Process for Driver Side Airbag. Proceedings of 21th Enhanced Safety of Vehicles (ESV) Conference, Paper No.09-0363, Stuttgart, Germany, June 15-18, 2009.

[22] Schenk, M., Viquerat, A.D., Seffen, K.A., and Guest, S.D. (2014). Review of Inflatable Booms for Deployable Space Structures: Packing and Rigidization. Journal of Spacecraft and Rockets, 51(3):762-778.

[23] Graczykowski, C. (2013). Theoretical Model and Numerical Methods for Adaptive Inflatable Structures. Proceeding of the Fourteenth International Conference on Civil, Structural and Environmental Engineering Computing, Stirlingshire, Scotland, 2013.

[24] Ha, W.P., Park S.J., and Spit H.H. (2004). Advanced Curtain Airbag Modeling Using the Uniform Pressure Approach Combined with a Gas Flow Analysis. SAE, 01-1632. 
[25] Kamiji, K., and Kawamura, N. (2001). Study of Airbag Interference with Out of Position Occupant by the Computer Simulation. Proceedings of the 17th International Technical Conference on the Enhanced Safety of Vehicles (ESV), Paper \#374, Amsterdam, Netherlands, June 4-7, 2001.

[26] Lee, E.S., and Youn, S.K. (2006). Finite Element Analysis of Wrinkling Membrane Structures with Large Deformations. Finite Element in Analysis and Design, 42:780-791.

[27] Abaqus User's Manual, Documentation Collection Version 6.14 (2014). Dassault Systemes Simulia Corp., Providence, RI.

[28] Altair HyperWorks 14.0, Altair HyperCrash: Altair HyperCrash Capabilities. Altair Engineering, Inc. Troy, MI.

[29] Wu, S.R., and Gu, L. (2012). Introduction to the Explicit Finite Element Method for Nonlinear Transient Dynamics. Wiley Press, September, 2012.

[30] Carlomagno, G.M. (2009), Elementi di Gasdinamica, Liguori, Naples, Italy.

[31] Kyle, B.G. (2000). Chemical Process Thermodynamics, 3rd Edition, Phi, Delhi, India.

[32] Zhang, L. (2016). Off-Axial Tensile Properties of Preconstraint PVDF Coated Polyester Fabric Under Different Tensile Rates Advances in Materials Science and Engineering, 2016, Article ID 9856474.

[33] Galliot, C., and Luchsinger, R.H. (2011). A New Test Method for the Investigation of Coated Fabric Shear Behavior. Tensinews, 20: 8-9.

[34] Cruz-Molina, J. (2008) Mechanical Characterization of Fabrics for Inflatable Structures, MS Thesis, Morgantown, WV.

[35] Air Properties Definitions. NASA, Glenn Research Center. https://www.grc.nasa.gov/www/k12/airplane/airprop.html last accessed on November 16, 2017.

[36] Della Pietra, A.R.G. (1990). Lezioni di Meccanica Applicata alle Macchine, Vol. 1. CUEN, Naples, Italy.

[37] Fermi, E. (1937). Thermodynamics. Reprint of the 1937 edition, Dover, New York, USA.

[38] Spectra® Fiber, Fishing Line, https://www.honeywell-spectra.com/applications/fishing-line/, last accessed on November 16, 2017.

[39] Martínez-Camacho A.P., Cortez-Rocha M.O., Graciano-Verdugo A.Z., Rodríguez-Félix F., CastilloOrtega M.M., Burgos-Hernández A., Ezquerra-Brauer J.M., Plascencia-Jatomea M., (2013). Extruded films of blended chitosan, low-density polyethylene, and ethylene acrylic acid, Carbohydrate Polymers, 91:666- 674.

[40] Salih S.E., Hamood A.F., Abd Al-salam A.H., (2013). Comparison of the Characteristics of LDPE: PP and HDPE: PP Polymer Blends, Modern Applied Science; Vol. 7, No. 3.

[41] Chowdhury S.R., Sabharwal S., (2011). Molecular-scale design of a high-performance organicinorganic hybrid with the help of gamma radiation, Journal of Materials Chemistry, 21, 6999. 
[42] Gao J., Chen F., Wang K., Deng H., Zhang Q., Bai H., Fu Q., (2011). A promising alternative to conventional polyethylene with poly(propylene carbonate) reinforced by graphene oxide nanosheets, Journal of Materials Chemistry, 21, 17627.

[43] Carotenuto G., De Nicola S., Palomba M., Pullini D., Horsewell A., Hansen T.W., Nicolais L., (2012). Mechanical properties of low-density polyethylene filled by graphite nanoplatelets, Nanotechnology 23, 485705 (8pp).

[44] Lopes C.M.A., Felisberti M.I., (2006). Composite of Low-Density Polyethylene and Aluminum Obtained from the Recycling of Postconsumer Aseptic Packaging, Journal of Applied Polymer Science.

[45] Elabbasi N., Bergstrom J., (2015). Constitutive Modeling of Polyethylene in COMSOL Multiphysics, COMSOL Conference 2015, Boston.

[46] Kaci M., Benhamida A., Cimmino S., Silvestre C., Carfagna C., (2005) Waste and Virgin LDPE/PET Blends Compatibilized with an Ethylene-Butyl Acrylate-Glycidyl Methacrylate (EBAGMA) Terpolymer, 1 Morphology and Mechanical Properties, Macromolecular Materials and Engineering.

[47] Plastics International, LPDE (Low-Density Polyethylene), www.plasticsintl.com.

[48] AZO Materials, Low-Density Polyethylene - LDPE, www.AZoM.com.

[49] Durmus A., Kasgoz A., Macosko C.W., (2008) Mechanical Properties of Linear Low-density Polyethylene (LLDPE)/clay Nanocomposites: Estimation of Aspect Ratio and Interfacial Strength by Composite Models, Journal of Macromolecular Science, Part B: Physics, 47:608-619.

[50] Xu M., Huang G., Feng S., McShane G.J., Stronge W.J., (2016). Static and Dynamic Properties of Semi-Crystalline Polyethylene, Polymers, 8, 77.

[51] WS Hampshire, Inc, Thermoplastic properties. http://catalog.wshampshire.com/Asset/plastics_properties_2.pdf

[52] RTP Co. Imagineering Plastics, RTP 700 A - Low-Density Polyethylene (LDPE) Unfilled, web.rtpcompany.com.

[53] Strapasson R., Amico S.C., Pereira M.F.R., Sydenstricker T.H.D., (2005). Tensile and impact behavior of polypropylene/low-density polyethylene blends, Polymer Testing 24 468-473.

[54] Molaei S., (2016). The measurement of Young's modulus of thin films using secondary laser speckle patterns, Measurement, 92:28-33. 